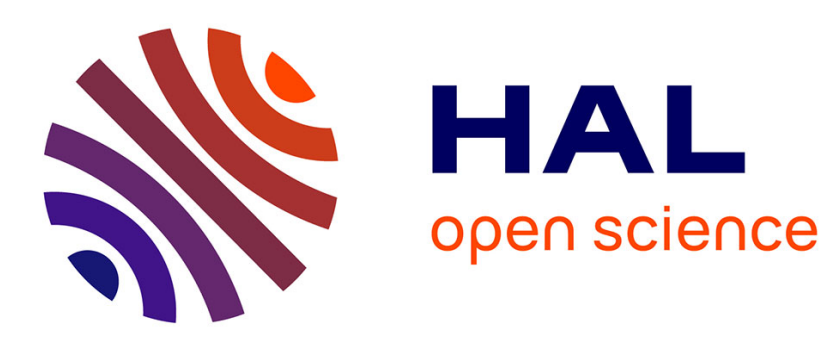

\title{
The performance of the hypergeometric $\mathrm{n} p$ chart with estimated parameter
}

Arne Johannssen, Nataliya Chukhrova, Philippe Castagliola

\section{To cite this version:}

Arne Johannssen, Nataliya Chukhrova, Philippe Castagliola. The performance of the hypergeometric $\mathrm{n}$ p chart with estimated parameter. European Journal of Operational Research, 2022, 296 (3), pp.873-899. 10.1016/j.ejor.2021.06.056 . hal-03505717

\section{HAL Id: hal-03505717 https://hal.science/hal-03505717}

Submitted on 31 Dec 2021

HAL is a multi-disciplinary open access archive for the deposit and dissemination of scientific research documents, whether they are published or not. The documents may come from teaching and research institutions in France or abroad, or from public or private research centers.
L'archive ouverte pluridisciplinaire HAL, est destinée au dépôt et à la diffusion de documents scientifiques de niveau recherche, publiés ou non, émanant des établissements d'enseignement et de recherche français ou étrangers, des laboratoires publics ou privés. 


\title{
The performance of the hypergeometric $n p$ chart with estimated parameter
}

\author{
Arne Johannssen ${ }^{1} \quad$ Nataliya Chukhrova $2^{2} \quad$ Philippe Castagliold $2^{3}$
}

\begin{abstract}
Although it is well known that the performance of attribute control charts decreases significantly when the assumption of known process parameters is invalid, this assumption is prevalent in the pertinent literature. However, in most practical applications, the process parameters have to be estimated from a finite in-control Phase I sample, and therefore the performance of attribute control charts should be evaluated from the perspective of estimated process parameters. In this paper, we compare the run length properties of the hypergeometric $n p$ chart in both the known and estimated parameter cases. In particular, we investigate the required number of Phase I samples and new specific chart parameters that allow the hypergeometric $n p$ chart with estimated parameter $p$ to have approximately the same in-control performance as in the known parameters case. Moreover, we perform a comprehensive in-control and out-of-control comparison of the hypergeometric $n p$ chart with its binomial counterpart. In order to achieve these objectives, we also present a new approach to effectively compute the probability distribution of the sum of independent and identically hypergeometric-distributed random variables. The proposed approximation reduces the computational effort to a few seconds while keeping a remarkable high accuracy with only negligible deviations compared to the exact distribution obtained via convolution.
\end{abstract}

Keywords: Quality control; attribute control charts; convolution; fraction nonconforming; run length properties

\section{Introduction}

Quality is an increasingly important decisive criterion for consumers to choose between different products or services. It is therefore of vital importance for all industries to produce quality products or offer quality services in order to remain competitive with their competitors. Processes that produce quality products can enable a manufacturing company to achieve good production yield, low production cost and products that exceed customer expectations. The area of Statistical Process Monitoring (SPM) provides a collection of powerful tools that employs statistical techniques to minimize the variability and ensures continuous quality improvement of processes (see e.g. Chong et al. 12 ). The most important tools in SPM are control charts, which are well-suited to support measuring, monitoring, and improving of various kinds of processes. They help to distinguish common/chance from special/assignable causes of variation as a guide for decision making, and processes can be enhanced to perform consistently and predictably for higher quality, lower cost, and higher effective capacity. Over the years, and especially in recent times, numerous articles have been published on control charts to optimize process monitoring through various improvements, amendments, and adaptations to current practical needs, see e.g. Teoh et al. 40], Mitra et al. [31], Filho \& Valk [18, Song et al. [37, 38, Nguyen et al. 35], just to name a few relevant works.

The majority of the literature on control charts is based on the assumption of known process parameters. In most practical situations, however, the process parameters are rarely known and they need to be estimated from a finite number of Phase I samples. It is therefore important to stress that the performance of control charts deteriorates significantly when the assumption of known parameters is invalid, due to the variability in parameter estimation during the in-control Phase I (see e.g. Chong et al. [12]). The problem of evaluating

\footnotetext{
${ }^{1}$ University of Hamburg, Hamburg, Germany, arne.johannssen@uni-hamburg. de (corresponding author).

${ }^{2}$ University of Hamburg, Hamburg, Germany, nataliya.chukhrova@uni-hamburg.de.

${ }^{3}$ Université de Nantes \& LS2N UMR CNRS 6004, Nantes, France, philippe.castagliola@univ-nantes.fr.
} 
the performance of control charts when the parameters are estimated has been investigated by many authors, mostly with a focus on continuous, location-type, control charts (see e.g. Jones et al. 24, Nedumaran \& Pignatiello [33, Chakraborti 7,, Zhang et al. 43, Hu et al. 21], Hu \& Castagliola 20, Motsepa et al. [32]) or dispersion-type control charts (see e.g. Maravelakis et al. [30, Maravelakis \& Castagliola 29, Castagliola et al. 4, Castagliola \& Maravelakis 2], Castagliola et al. [5]). Recently, there are also a lot of works focusing on the effects of parameter estimation on the performance of control charts that are worth to be mentioned, e.g. Zwetsloot \& Woodall [4] (conditional performance of control charts), Chong et al. [10, 11] (optimal design of modified group runs charts), Hu et al. 22, Khoo et al. 25, Tang et al. 39. (design of EWMA median charts), and Lee et al. 28] (economic-statistical design of synthetic $n p$ charts). For literature reviews on the impact of parameter estimation on control charts performance we refer to Jensen et al. 23] and Psarakis et al. [36].

Since many quality characteristics can be classified as either conforming or nonconforming, attribute control charts such as $p$ charts (for fraction of nonconforming units), $n p$ charts (for number of nonconforming units), $c$ charts (for number of nonconformities), and $u$ charts (for number of nonconformities per unit) are commonly used for monitoring attribute process data. Considering the performance of attribute control charts with estimated process parameters, there are relatively few articles to date (to the best of the authors' knowledge), e.g. Braun [1, Chakraborti \& Human [8, 9], Castagliola \& Wu [3, Tiplica 41, Hashemian et al. [19, Wu et al. 42, Faraz et al. [17, Lee \& Khoo [27. These papers deal with the derivation of run length properties for (Poisson) $c$ charts as well as (binomial) $p$ and $n p$ charts when the process parameters are estimated. However, all these papers (with the exception of Tiplica [11), only focus on the in-control analysis and they do not propose a complete picture by also investigating the out-of-control performance of the corresponding control charts.

Recently, $p$ charts based on the hypergeometric distribution have been proposed to account for the population size effect in periodical processes (see Chukhrova \& Johannssen [13, 14]). These charts have a series of benefits including the following:

- They can be used for monitoring continuous and periodical processes.

- They take fluctuations of the underlying process into account.

- They do not require a constant probability being nonconforming for successively sampled units.

- They do not presume independence of successively sampled units.

- They are able to monitor processes with an infinite or a finite horizon.

In particular, Chukhrova \& Johannssen 13 introduced the hypergeometric $p$ chart with Shewhart-type control limits in the known parameter case, discussed the operating characteristic function with respect to the type I and II errors linked to the average run length (ARL), studied the impact of the sample and population size on the center line and the control limits, provided guidelines of specifying the appropriate sample size for specific scenarios and gave a practical application including comparative analysis with the binomial $p$ chart. In addition, Chukhrova \& Johannssen [14] presented the hypergeometric $p$ chart with probability control limits, in turn in the known parameter case, performed a comprehensive sensitivity analysis in relation to the control limits of both types considering changes in all relevant parameters, gave a brief sensitivity analysis in relation to the ARL, and provided a practical application as well as a comparative study with the binomial $p$ chart.

That is, hypergeometric $p$ charts have been considered only in the case of known parameter and there is the need to extend the analysis to the more realistic (and thus more important) unknown parameter case and to study the effect of the parameter estimation on the control charts performance. To fill this gap, we introduce the hypergeometric $n p$ chart and discuss both the known and the unknown parameter case. The $n p$ chart 
has the benefit that it deals with integer values, while the $p$ chart deals with numbers that are not integer, neither real (even if one treat them as real numbers), i.e., $n p$ charts are more convenient to handle in practical applications. Further, we conduct a comprehensive in-control and out-of-control performance analysis of the hypergeometric $n p$ chart in both the known and estimated parameter case, that is, we investigate the overall impact of the parameter estimation on the control charts performance. We also compare these results with the results obtained for the binomial $n p$ chart, extending in this way the previous literature by performing an out-of-control analysis for the benchmark chart. Moreover, to increase the practical applicability of the proposed hypergeometric $n p$ chart, we investigate the required number of Phase I samples and provide alternative chart parameters in the estimated parameter case to have a similar in-control ARL as the one obtained when the parameter $p$ is known.

To achieve these several objectives, we need to compute the probability distribution of the sum of independent and identically hypergeometric-distributed random variables. While convoluting random variables following either a Poisson or a binomial distribution is straightforward, convoluting hypergeometric-distributed random variables is not. To overcome this problem, we also propose a new approximation for the distribution of the sum of independent and identically hypergeometric-distributed random variables. This approximation has remarkable properties and may be helpful - beyond the problem discussed in this paper - in all areas, where the problem of convoluting hypergeometric-distributed random variables occurs.

The paper is organized as follows. In Section 2 , we introduce the hypergeometric $n p$ chart in both the known and the estimated parameter cases and, moreover, we give a brief scheme of the implementation steps for these control charts in practical applications. In Section 3, we propose exact and approximate approaches for achieving the probability distribution of the sum of independent and identically hypergeometric-distributed random variables. Afterward, in Section 4 we perform both an in- and out-of-control analysis of the hypergeometric $n p$ chart in the known and the estimated parameter cases, and carry out comparisons with the binomial $n p$ chart. Section 5 presents an illustrative example considering the known and the unknown parameter case. In Section 6, concluding remarks are given.

\section{The hypergeometric $n p$ chart}

\subsection{The case of known parameter $p_{0}$}

Let us assume that $Y_{i}, i=1,2, \ldots, k$, are $k$ Phase II independent random variables corresponding to the number of nonconforming units obtained after sampling without replacement $n$ units in a population of size $N>n, n, N \in \mathbb{N}^{*}$, containing an unknown proportion $p_{1} \in[0,1]$ of nonconforming units. By definition, $Y_{i}$, $i=1,2, \ldots, k$, are hypergeometric random variables of parameters $\left(N, n, p_{1}\right)$, defined on $\left\{y_{\min }=\max (0, n-\right.$ $\left.\left.N\left(1-p_{1}\right)\right), \ldots, y_{\max }=\min \left(N p_{1}, n\right)\right\}$. Similarly to Chukhrova \& Johannssen [13, 14, these random variables can be monitored using a hypergeometric $n p$ chart with the following Shewhart-type control limits

$$
\begin{aligned}
\mathrm{LCL} & =\max \left\{0,\left\lceil n p_{0}-K \sqrt{n p_{0}\left(1-p_{0}\right) \frac{N-n}{N-1}}\right\rceil\right\}, \\
\mathrm{UCL} & =\left\lfloor n p_{0}+K \sqrt{n p_{0}\left(1-p_{0}\right) \frac{N-n}{N-1}}\right\rfloor,
\end{aligned}
$$

where $\lceil\cdots\rceil$ and $\lfloor\cdots\rfloor$ denote the rounded up and rounded down integer, $p_{0}$ is the known in-control proportion of nonconforming units and $K>0$ is a constant to be fixed. Therefore, the probability $\theta$ that the number of nonconforming units $Y_{i}, i=1,2, \ldots, k$, is outside the control limits is equal to

$$
\theta=1-F_{\mathrm{HYP}}\left(\mathrm{UCL} \mid N, n, p_{1}\right)+F_{\mathrm{HYP}}\left(\mathrm{LCL}-1 \mid N, n, p_{1}\right),
$$


where $F_{\mathrm{HYP}}(y \mid N, n, p)$ is the c.d.f. of the hypergeometric distribution of parameters $(N, n, p)$, i.e.,

$$
F_{\mathrm{HYP}}(y \mid N, n, p)=\sum_{x=y_{\min }}^{y} \frac{\left(\begin{array}{c}
\lfloor p \downarrow\rfloor \\
x
\end{array}\right)\left(\begin{array}{c}
\lceil N(1-p)\rceil \\
n-x
\end{array}\right)}{\left(\begin{array}{l}
N \\
n
\end{array}\right)} .
$$

The run length $\mathrm{RL}$ of the hypergeometric $n p$ chart is a geometric random variable of parameter $\theta$, i.e., the p.m.f. and the c.d.f. of RL are defined for $\ell \in\{1,2, \ldots\}$ and they are equal to $f_{\mathrm{RL}}(\ell)=\theta(1-\theta)^{\ell-1}$ and $F_{\mathrm{RL}}(\ell)=1-(1-\theta)^{\ell}$, respectively, and its first two moments are ARL $=\mathbb{E}[\mathrm{RL}]=\frac{1}{\theta}$ and $\mathrm{SDRL}=\sigma_{\mathrm{RL}}=\frac{\sqrt{1-\theta}}{\theta}$.

\subsection{The case of estimated parameter $p_{0}$}

If the in-control proportion $p_{0}$ of nonconforming units is unknown, it has to be estimated from $m$ Phase I independent random variables $X_{i}, i=1, \ldots, m$, corresponding to the number of nonconforming units obtained after sampling without replacement $n$ units in an in-control population of size $N>n$ (i.e. containing a proportion $p_{0} \in[0,1]$ of nonconforming units). By definition, $X_{i}, i=1, \ldots, m$, are hypergeometric random variables of parameters $\left(N, n, p_{0}\right)$ defined on $\left\{x_{\min }=\max \left(0, n-N\left(1-p_{0}\right)\right), \ldots, x_{\max }=\min \left(N p_{0}, n\right)\right\}$. An estimator $\hat{p}_{0}$ of $p_{0}$ is given by

$$
\hat{p}_{0}=\frac{1}{m n} \sum_{i=1}^{m} X_{i}=\frac{X}{m n} .
$$

Since the estimator (2.3) is the sample mean, it is the best linear unbiased estimator (BLUE) of $p_{0}$ and satisfies the properties of consistency and efficiency [34].

However, $X$ does in general not follow a hypergeometric distribution, apart from some special cases: $m=1$ or $n=N$ or $p_{0} \in\{0,1\}$. Thus, we propose an appropriate method to achieve the probability distribution $f_{X}\left(x \mid m, n, N, p_{0}\right)$ of $X$ in Section 3 .

When $p_{0}$ is estimated by $\hat{p}_{0}$, the Shewhart-type control limits of the hypergeometric $n p$ chart become

$$
\begin{aligned}
& \widehat{\mathrm{LCL}}=\max \left\{0,\left\lceil n \hat{p}_{0}-K \sqrt{n \hat{p}_{0}\left(1-\hat{p}_{0}\right) \frac{N-n}{N-1}}\right\rceil\right\}, \\
& \widehat{\mathrm{UCL}}=\left\lfloor n \hat{p}_{0}+K \sqrt{n \hat{p}_{0}\left(1-\hat{p}_{0}\right) \frac{N-n}{N-1}}\right\rfloor .
\end{aligned}
$$

Let $\widehat{\theta}$ be the probability that the number $Y_{i}$ of nonconforming units in the $i$-th sample is outside the conditional control limits (that is, control limits $\widehat{\mathrm{LCL}}$ and $\widehat{\mathrm{UCL}}$ defined conditionally to $X=x$ ), i.e.,

$$
\widehat{\theta}=1-P\left(Y_{i} \leq \widehat{\mathrm{UCL}} \mid X=x\right)+P\left(Y_{i}<\widehat{\mathrm{LCL}} \mid X=x\right)
$$

Replacing $\widehat{\mathrm{LCL}}$ and $\widehat{\mathrm{UCL}}$ in $(2.6)$ by $(2.4)$ and $(2.5)$, respectively, we get

$$
\begin{aligned}
\widehat{\theta}= & 1-P\left(Y_{i} \leq\left\lfloor n \hat{p}_{0}+K \sqrt{n \hat{p}_{0}\left(1-\hat{p}_{0}\right) \frac{N-n}{N-1}}\right\rfloor \mid X=x\right) \\
& +P\left(Y_{i} \leq\left\lceil n \hat{p}_{0}-K \sqrt{n \hat{p}_{0}\left(1-\hat{p}_{0}\right) \frac{N-n}{N-1}}\right\rceil-1 \mid X=x\right) .
\end{aligned}
$$


Replacing $\hat{p}_{0}$ in 2.7 by $\frac{X}{m n}$ (given by 2.3 ) directly leads to

$$
\begin{aligned}
\widehat{\theta}= & 1-P\left(Y_{i} \leq\left\lfloor n \frac{X}{m n}+K \sqrt{n \frac{X}{m n}\left(1-\frac{X}{m n}\right) \frac{N-n}{N-1}}\right\rfloor X=x\right) \\
& +P\left(Y_{i} \leq\left\lceil n \frac{X}{m n}-K \sqrt{n \frac{X}{m n}\left(1-\frac{X}{m n}\right) \frac{N-n}{N-1}}\right\rceil-1 \mid X=x\right)
\end{aligned}
$$

that can be simplified to

$$
\begin{aligned}
\widehat{\theta}= & 1-P\left(Y_{i} \leq\left\lfloor\frac{x}{m}+K \sqrt{\frac{x}{m}\left(1-\frac{x}{m n}\right) \frac{N-n}{N-1}}\right\rfloor\right) \\
& +P\left(Y_{i} \leq\left\lceil\frac{x}{m}-K \sqrt{\frac{x}{m}\left(1-\frac{x}{m n}\right) \frac{N-n}{N-1}}\right\rceil-1\right) .
\end{aligned}
$$

Since $Y_{i} \sim \operatorname{HYP}\left(N, n, p_{1}\right)$, we have

$$
\begin{aligned}
\widehat{\theta}= & 1-F_{\mathrm{HYP}}(\overbrace{\left\lfloor\frac{x}{m}+K \sqrt{\frac{x}{m}\left(1-\frac{x}{m n}\right) \frac{N-n}{N-1}} \mid\right.}^{\text {conditional UCL }} \mid N, n, p_{1}) \\
& +F_{\mathrm{HYP}}(\underbrace{\left[\frac{x}{m}-K \sqrt{\frac{x}{m}\left(1-\frac{x}{m n}\right) \frac{N-n}{N-1}} \mid\right.}_{\text {conditional LCL }}-1 \mid N, n, p_{1})
\end{aligned}
$$

Let RL be the run length of the hypergeometric $n p$ chart with estimated parameter $p_{0}$. Since $X \sim \sum_{i=1}^{m} X_{i}$ is a random variable of parameters $\left(m, n, N, p_{0}\right)$ defined on $x \in\left\{m x_{\min }, \ldots, m x_{\max }\right\}$, the (unconditional) p.m.f. and c.d.f. of RL are

$$
\begin{aligned}
& f_{\mathrm{RL}}(\ell)=\sum_{x=m x_{\min }}^{m x_{\max }} f_{X}\left(x \mid m, n, N, p_{0}\right) \widehat{\theta}(1-\widehat{\theta})^{\ell-1} \\
& F_{\mathrm{RL}}(\ell)=1-\sum_{x=m x_{\min }}^{m x_{\max }} f_{X}\left(x \mid m, n, N, p_{0}\right)(1-\widehat{\theta})^{\ell}
\end{aligned}
$$

where $f_{X}\left(x \mid m, n, N, p_{0}\right)$ is the p.m.f. of $X$ (see Section 3 for computational details). The (unconditional) ARL and SDRL of the hypergeometric $n p$ chart with estimated parameter $p_{0}$ are then defined by

$$
\mathrm{ARL}=\sum_{x=m x_{\min }}^{m x_{\max }} f_{X}\left(x \mid m, n, N, p_{0}\right)\left(\frac{1}{\widehat{\widehat{\theta}}}\right)
$$

and

$$
\mathrm{SDRL}=\sqrt{\mathbb{E}\left[\mathrm{RL}^{2}\right]-\mathrm{ARL}^{2}}
$$

respectively, where

$$
\mathbb{E}\left[\mathrm{RL}^{2}\right]=\sum_{x=m x_{\min }}^{m x_{\max }} f_{X}\left(x \mid m, n, N, p_{0}\right)\left(\frac{2-\widehat{\theta}}{\widehat{\theta}^{2}}\right)
$$


Remark 2.1 To allow adequate comparisons with the performance measures in the known parameter case (given $p_{0}=\widehat{p}_{0}$ ), the calculation of ARL and SDRL in the estimated parameter case should be performed under the condition of reasonable control limits. Due to the nature of Shewhart-type control limits, the (conditional) probability of a violation of the control limits can be equal to zero either for both cases (i.e., $\theta=\widehat{\theta}=0$ ) or

only for the estimated parameter case (i.e., $\hat{\theta}=0$ for some values of $x$, see (2.8)) that results in infinitely large values of ARL and SDRL. While in the first scenario both charts are equivalent, in the second scenario the performance measures of the hypergeometric np chart with estimated parameter $p_{0}$ would be biased. To overcome this bias, we propose to use $\widehat{\mathrm{UCL}}$ given by $(2.5$ ) (which corresponds to UCL in the known parameter case, see (2.2) instead of conditional UCL (see (2.8) for affected values of $x$ in the calculation of $\widehat{\theta}$. This procedure reduces the bias in an appropriate way by considering $\widehat{\theta}>0$ instead of $\widehat{\theta}=0$, i.e., by implementing a reasonable conditional upper control limit $\mathrm{UCL}<\min \left(N \widehat{p}_{0}, n\right)$ and $\mathrm{UCL}<\min \left(N p_{1}, n\right)$, respectively.

\subsection{Implementation of the proposed methods in practical applications}

In this section we give a brief scheme of the basic implementation steps for the $n p$ chart in practical applications:

(1) Choose the appropriate $n p$ chart

(a) by specifying whether the population size is finite or infinite and

(b) by determining whether the known or estimated parameter case is present

(2) Calculate the center line and the control limits of the selected $n p$ chart

(3) Compute in-control performance measures $\mathrm{ARL}_{0}$ and $\mathrm{SDRL}_{0}$ (where $p_{1}=p_{0}$ )

(4) Compute out-of-control performance measures $\mathrm{ARL}_{1}$ and $\mathrm{SDRL}_{1}$ (where $p_{1}=\tau p_{0}$ with $\tau>1$ )

To make the above implementation procedure illustrative, we provide a decision tree in Figure 1 with visualization of all implementation steps on the one hand, and on the other hand we give a detailed description of the individual steps in the following.

The first criterion (see step (1a)) for selecting the appropriate $n p$ chart is the type of the underlying process, i.e., whether the process to be monitored is continuous or periodical in nature. If the process is continuous, the population size is considered as infinitely large, while a periodical process implies a finite population size. To correctly account for the effect of a finite population size in periodical processes, the hypergeometric $n p$ chart must be chosen, otherwise (if the population size is infinitely large) the common binomial $n p$ chart can be used.

The second criterion (see step (1D) for choosing the appropriate $n p$ chart is given by the knowledge of process parameter $p_{0}$. The parameter $p_{0}$ can either be specified by the practitioner/researcher, for instance, by making reference to a nominal value or to a retrospective study on empirical data (known parameter case), or it should be estimated from retrospective process data (estimated parameter case). In the second case, the $n p$ chart must be implemented in two consecutive phases (see, e.g., Celano \& Chakraborti [6]),

- Phase I: a preliminary retrospective study is carried out to bring the process to the in-control state and to get a reliable estimation of the process parameter $p_{0}$,

- Phase II: on-line process monitoring with the aim of checking the process stability during production,

while in the first case, the $n p$ chart can be implemented directly for on-line process monitoring (Phase II).

Next, the center line and the control limits have to be computed in the first place for on-line process monitoring in both cases (see step (2)). In the second place, they also serve as the basis for the computation of 
performance measures in the known parameter case, while conditional control limits (see (2.8) ) are used for the calculation of single values of $\widehat{\theta}$, conditionally to $X=x$, in the estimated parameter case.

Finally, specifying the fraction nonconforming $p_{1}=p_{0}$ or $p_{1}=\tau p_{0}$ with $\tau>1$, i.e., as in-control or out-ofcontrol fraction nonconforming, $\mathrm{ARL}_{0}$ and $\mathrm{SDRL}_{0}$ or $\mathrm{ARL}_{1}$ and $\mathrm{SDRL}_{1}$ can be calculated to make statements regarding process stability (see step (3)) and/or shifts in the process quality level (see step (4)). 


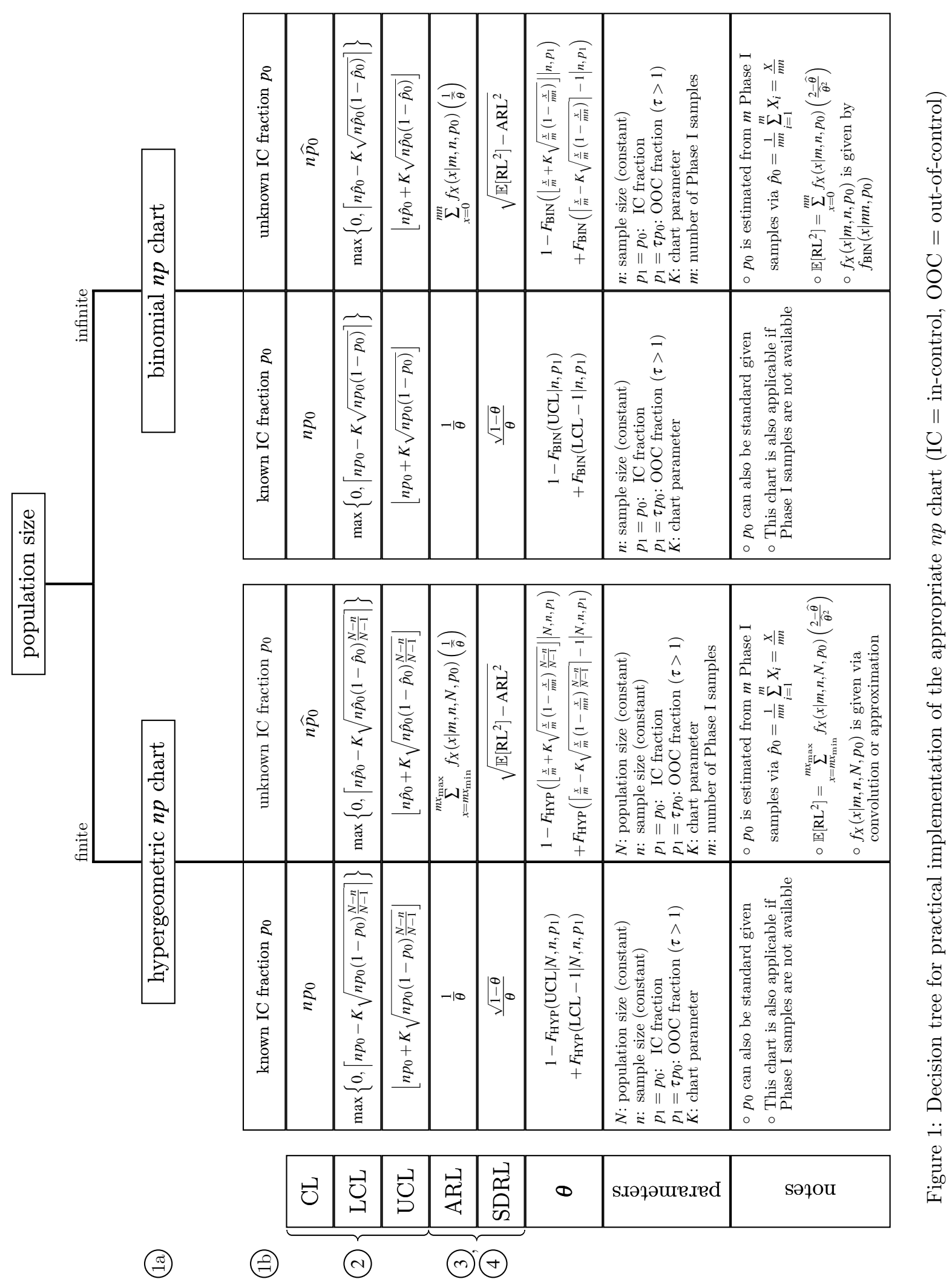




\section{Determining the probability distribution of $X$}

\subsection{Convolution}

As a matter of fact, the random variable $X=\sum_{i=1}^{m} X_{i}$ is in general not hypergeometric-distributed. Thus, we need a practicable way to determine its probability distribution. Since $X_{1}, \ldots, X_{m}$ are i.i.d. random variables, this can be achieved by convolution.

Convolution is a direct method to calculate the probability distribution of $X$. The probability distribution of the sum of i.i.d. hypergeometric distributed random variables $X_{i}, i=1, \ldots, m$, is the convolution of their individual distributions. Some well known distributions have simple convolutions, e.g. binomial, geometric, Poisson, but unfortunately there is no simple convolution with regard to the hypergeometric distribution.

Regarding the hypergeometric case, the $m$-fold convolution of $F_{X_{i}}$ and $f_{X_{i}}$ can be obtained using the following recursive equations (based on general convolution equations, see e.g. Dickson [16])

$$
\begin{aligned}
& F_{X}(s)=F_{X_{i}}^{(m)}(s)=\underbrace{F_{X_{i}} \circledast \cdots \circledast F_{X_{i}}(s)}_{\times m}=\sum_{j=m x_{\min }}^{s} F_{X_{i}}^{(m-1)}(s-j) f_{X_{i}}(j) \\
& f_{X}(s)=f_{X_{i}}^{(m)}(s)=\underbrace{f_{X_{i}} \circledast \cdots \circledast f_{X_{i}}(s)}_{\times m}=\sum_{j=m x_{\min }}^{s} f_{X_{i}}^{(m-1)}(s-j) f_{X_{i}}(j)
\end{aligned}
$$

where

$$
\begin{aligned}
& F_{X_{i}}^{(0)}(s)=1 \quad \text { and } \quad F_{X_{i}}^{(1)}=F_{X_{i}}
\end{aligned}
$$

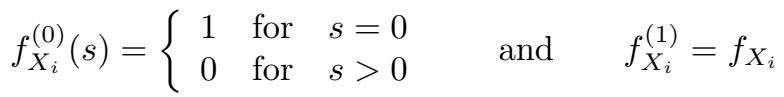

and $s \in\left\{m x_{\min }, \ldots, m x_{\max }\right\}$. Note that the order of convolution does not matter. Since there are no closed form solutions for $F_{X}$ and $f_{X}$, the analytical calculation of $F_{X}$ and $f_{X}$ using convolution is impossible. In addition, the numerical calculation of $F_{X}$ and $f_{X}$ with help of (3.1) and (3.2), respectively, requires a very high computational effort for combinations of higher values of $n(n \geq 100)$ and $m(m \geq 1000)$.

\subsection{The algorithm of De Pril [15]}

As for the computation of $F_{X}$ and $f_{X}$ using smaller values of $n$ and $m$, i.e., $n \leq 50$ and $m \leq 100$, there are recursive algorithms that can be utilized to calculate $F_{X}$ and $f_{X}$ significantly faster (while keeping a very high accuracy). The algorithm of De Pril [15] is such an efficient algorithm:

$$
f_{X_{i}}^{(m)}(s)=P(X=s)= \begin{cases}\left(f_{X_{i}}(0)\right)^{m} & \text { if } s=0 \\ \frac{1}{f_{X_{i}}(0)} \sum_{j=1}^{s}\left((m+1) \frac{j}{s}-1\right) f_{X_{i}}(j) P(X=s-j) & \text { if } s \in \mathbb{N}^{*}\end{cases}
$$

where $X_{i}$ are i.i.d. (hypergeometric) random variables with $f_{X_{i}}(0) \neq 0, i=1, \ldots, m$. Since we are interested in low values of $p(p \ll 1)$ in statistical process monitoring, $x_{\min }=0$ is usually satisfied. Therefore, the algorithm of De Pril [15] can be used in its standard form given above (for $x_{\min }>0$ see the algorithm in shifted form in De Pril [15]).

However, for mid-sized values of $m(100<m<1000)$ the results of the algorithm by De Pril [15] converge to zero due to the fact that $\left(f_{X_{i}}(0)\right)^{m}=0$ using standard settings of statistical software, i.e., double-precision instead of variable-precision floating point numbers. On the other hand, the computation of $F_{X}$ and $f_{X}$ with variable-precision floating point numbers is very extensive for $m \geq 1000$ and thus can not be recommended for 
the user. A further problem results in turn from above mentioned standard settings and is related to higher values of $n$ or $p$, which entail substantial errors caused by numerous multiplication operations together with single infinitesimal hidden round-off errors and thus provide unreliable results. Summarized, the algorithm by De Pril [15] is appropriate for calculation of $f_{X}$ and $F_{X}$ using combinations of smaller values of $m, n, p$, i.e., $m \leq 100, n \leq 50, p \leq 0.1$.

\subsection{A new approximation}

In order to find a remedy in cases with higher values of $m, n$ or $p$, we suggest to use a new approximation of $F_{X}$ and $f_{X}$ which is not based on the algorithm by De Pril [15]. This approximation considerably simplifies the calculation of $F_{X}$ and $f_{X}$, since the sum of i.i.d. hypergeometric distributed random variables $X_{i}, i=$ $1, \ldots, m$, is approximated by a hypergeometric-distributed random variable $Z$ of parameters $\left(m N, m n, p_{0}\right)$, i.e.,

$$
\begin{aligned}
f_{X}(s) & \approx f_{\mathrm{HYP}}\left(x \mid m N, m n, p_{0}\right) \\
F_{X}(s) & \approx F_{\mathrm{HYP}}\left(x \mid m N, m n, p_{0}\right)
\end{aligned}
$$

Using this approximation, the time required for the computation of $F_{X}$ and $f_{X}$ can be reduced to a few seconds while keeping a remarkable high accuracy. It also allows to approximate the (unconditional) ARL and SDRL in 2.10) and 2.11). Moreover, numerical simulation results show that the higher the values of $N, n$ and $m$, the better the approximation (see details in Section 4 ).

\section{Numerical analysis}

\subsection{In-control performance}

In Tables 28 and Table 9 , the in-control ARL and SDRL values (i.e., $\mathrm{ARL}_{0}$ and $\mathrm{SDRL}_{0}$ ) are presented for the hypergeometric $n p$ chart and the binomial $n p$ chart, respectively, given $K=3$ as well as

$$
\begin{aligned}
N & \in\{100,200,500,1000,2000,5000,10000\} & & \text { (see Tables 28) } \\
n & \in\{25,50,75,100\} & & \text { (see blocks in Tables 29) } \\
m & \in\{10,20,50,100,200,1000, \infty\} & & \text { (see columns in Tables 29) } \\
p_{0} & \in\{0.01,0.02,0.05,0.10,0.15,0.20\} & & \text { (see rows in Tables 2 9) }
\end{aligned}
$$

The above specified values of the parameters $N, n, m, p_{0}$ were chosen for the following reasons:

- The values of $N$ show the transition from a finite to an infinite population in Phase I and II.

- The values of $n$ are common attribute-type sample sizes in Phase I and II.

- The finite values of $m$ imply the case of estimated parameter $p_{0}$ from a Phase I sample and allow to consider different Phase I lengths (short, middle, long), while $m \rightarrow \infty$ corresponds to the case of known parameter $p_{0}$ in Phase II.

- The values of $p_{0}$ show the transition from low to larger fractions nonconforming. They can be estimated from a Phase I sample using estimator 2.3.

It should be noted that the in-control values for the hypergeometric $n p$ chart are calculated using the approximation of $X=\sum_{i=1}^{m} X_{i}$ via $Z \sim \operatorname{HYP}\left(m N, m n, p_{0}\right)$ discussed in Section 3.3 for $m \in\{10,20,50,100,200,1000\}$ (estimated parameter case) under the condition of reasonable control limits (see Remark 2.1). As for $m \rightarrow \infty$ (known parameter case), the in-control values for the hypergeometric $n p$ chart are obtained by using exact hypergeometric distribution (see Section 2.1). 
The reasons for using the approximate results are as follows. We have conducted comprehensive comparisons of the hypergeometric and approximate hypergeometric $n p$ charts and found that the values of exact $\mathrm{ARL}_{0}$ and $\mathrm{SDRL}_{0}$ are throughout approximately equal to the respective values of approximate $\mathrm{ARL}_{0}$ and $\mathrm{SDRL}_{0}$, and there is (i) no tendency for a systematic deviation ( $A R L_{H Y P}-\mathrm{ARL}_{\mathrm{HYPappr}}$, $\mathrm{SDRL}_{\mathrm{HYP}}-\mathrm{SDRL}_{\mathrm{HYPappr}}$.) to one direction regarding $n$, (ii) a negative tendency regarding $p_{0}$ for smaller values of $N(N \leq 100)$, and (iii) a positive tendency regarding $m$ and smaller values of $N$. Moreover, when $N, n, m$ or $p_{0}$ increases, the difference between exact and approximate values for $\mathrm{ARL}_{0}, \mathrm{SDRL}_{0}$ decreases in a monotonic way and seems to converge to zero. For instance, the maximum of the relative deviation regarding $\mathrm{ARL}_{0}\left(\frac{\left|\mathrm{ARL}_{\mathrm{HYP}}-\mathrm{ARL}_{\mathrm{HYPappr}}\right|}{\mathrm{ARL}_{\text {HYP }}} \cdot 100 \%\right)$ is $2.21 \%$ for $N=100$ and $0.07 \%$ for $N=10000$. We mostly observe relative deviations regarding $\mathrm{ARL}_{0}$ that are $<0.1 \%$ for $N \leq 1000$ and $<0.05 \%$ for $N>1000$. Due to the excellent approximation accuracy and with the purpose of reducing the number and size of the tables, we have refrained from explicitly reporting the exact results in this paper.

We have also verified the obtained results in Tables 28 by conducting an additional simulation study. In particular, for selected combinations of $\left(m, N, n, p_{0}, \tau=1, K=3\right)$, we have computed $\mathrm{ARL}_{0}$ in the estimated parameter case via $\mathrm{ARL}_{0}^{\text {sim }}=\frac{\mathrm{ARL}_{0}^{(1)}+\mathrm{ARL}_{0}^{(2)}+\ldots+\mathrm{ARL}_{0}^{(s)}}{s}$ by repeating the following two steps $s=10^{3}$ times:

1. Generate a vector of $m$ hypergeometric random variables $X_{1}, \ldots, X_{n}$ of parameters $\left(N, n, p_{0}\right)$ and compute $\widehat{p}_{0}, \widehat{\mathrm{LCL}}, \widehat{\mathrm{UCL}}$ using $(2.3), \widehat{2.4}, 2.2 .5$. Recalculate this step if $\widehat{\mathrm{LCL}}=0 \cup\left(\widehat{\mathrm{UCL}} \geq \min \left(N \widehat{p}_{0}, n\right)\right) \cup$ $\left(\widehat{\mathrm{UCL}}<\min \left(N p_{0}, n\right)\right)$, i.e., in line with the approach of reasonable control limits.

2. Generate a vector of $r=10^{6}$ hypergeometric random variables $Y_{1}, \ldots, Y_{r}$ of parameters $\left(N, n, p_{1}\right)$ with $p_{1}=\tau p_{0}$, calculate $\widehat{\theta}=\frac{\#\left\{\left(Y_{i}<\widehat{\mathrm{LCL}}\right) \cup\left(Y_{i}>\widehat{\mathrm{UCL}}\right)\right\}}{r}$ and compute simulated $\mathrm{ARL}_{0}=\frac{1}{\hat{\theta}}$.

Selected results of the simulation study are shown in Table 10. As expected, the smaller the exact values of $\mathrm{ARL}_{0}$, the smaller the absolute deviations of the simulated values from the exact ones. The mean percentage deviation between exact and simulated values of $\mathrm{ARL}_{0}$ is around $1 \%$, thus confirming the accuracy of the results obtained using the exact formulas.

Analyzing Tables 2 9, we obtain the following results:

(1) Estimated parameter case (finite values of $m$ ):

- An increase in $N$ mostly leads to a decrease of $\mathrm{ARL}_{0}$ and $\mathrm{SDRL}_{0}$.

- An increase in $n$ provides as a tendency an increase of $\mathrm{ARL}_{0}$ and $\mathrm{SDRL}_{0}$, but with some exceptions (in particular when $N \leq 200$ or $p_{0} \geq 0.15$ ).

- An increase in $m$ mostly leads to a decrease of $\mathrm{ARL}_{0}$ and $\mathrm{SDRL}_{0}$, but with some exceptions (in particular when $p_{0} \geq 0.15$ and/or $N \leq 200$ ).

- An increase in $p_{0}$ up to 0.1 mostly implies an increase of $\mathrm{ARL}_{0}$ and $\mathrm{SDRL}_{0}$, while a further increase in $p_{0}$ leads as a tendency to a decrease of $\mathrm{ARL}_{0}$ and $\mathrm{SDRL}_{0}$. Moreover, there is as a rule a decreasing behavior of $\mathrm{ARL}_{0}$ and $\mathrm{SDRL}_{0}$ for lower values of $m(m \leq 20)$ in combination with $n \geq 75$ when $p_{0}$ increases, but an increasing behavior for larger values of $m$ and any values of $n$.

(2) Known parameter case $(m \rightarrow \infty)$ : The conclusions are similar as obtained in (1), except for the results regarding $m$ and $p_{0}$. In particular, when $p_{0}$ increases $\mathrm{ARL}_{0}$ and $\mathrm{SDRL}_{0}$ can increase or decrease.

(3) Comparison of the estimated and known parameter cases:

- The values of $\mathrm{ARL}_{0}$ and $\mathrm{SDRL}_{0}$ differ in general in the known and in the estimated parameter case. In particular, $\mathrm{ARL}_{0}$ and $\mathrm{SDRL}_{0}$ in the estimated parameter case can be either larger or smaller compared to the known parameter case, i.e., there is no clear tendency for positive or negative differences. 
- When $N, n, m$ or $p_{0}$ increases, the difference in terms of $\mathrm{ARL}_{0}$ (between the known and estimated parameter case) tends to decrease but not always in a monotonic way.

- For $N=n=100$, we have a total inspection of the population and consequently infinitely large values of $\mathrm{ARL}_{0}$ and $\mathrm{SDRL}_{0}$ in both cases due to the value of the type I error that is equal to zero. In addition, for $N \in\{100,200\}$ and $p_{0} \in\{0.01,0.02\}$, the values of $\mathrm{ARL}_{0}$ and $\mathrm{SDRL}_{0}$ can in turn be infinitely large (see Tables 23 ). The reason for these exceptions is given by the fact that these parameter combinations do not ensure reasonable control limits, and thus the (conditional) probability of a violation of the control limits is equal to zero for some values of $x$ in the estimated parameter case as well as throughout in the known parameter case.

(4) Comparison of the hypergeometric and binomial $n p$ charts (estimated parameter case):

- The values of $\mathrm{ARL}_{0}$ and $\mathrm{SDRL}_{0}$ are mostly larger with regard to the hypergeometric $n p$ chart compared to the binomial $n p$ chart, with only a few exceptions (for $N \leq 200$ ).

- As a consequence of an increase in $N$, the difference between $\mathrm{ARL}_{0}, \mathrm{SDRL}_{0}$ of both charts decreases and converges to zero.

- There is the tendency of a decreasing difference between $\mathrm{ARL}_{0}, \mathrm{SDRL}_{0}$ of both charts when $m$ or $p_{0}$ increases, but with a few exceptions.

- In contrast, there is no clear tendency regarding the difference between $\mathrm{ARL}_{0}, \mathrm{SDRL}_{0}$ of both charts for larger values of $N(N \geq 2000)$ when $n$ changes. For smaller values of $N(N \leq 1000)$ this difference seems to increase with an increasing $n$.

(5) Comparison of the hypergeometric and binomial $n p$ charts (known parameter case):

- The values of $\mathrm{ARL}_{0}$ and $\mathrm{SDRL}_{0}$ are mostly larger in the hypergeometric case compared to the binomial case, with only few exceptions (mostly for $n=25,100$ ).

- For an increasing value of $N$, the absolute difference between $\mathrm{ARL}_{0}, \mathrm{SDRL}_{0}$ for both charts decreases and converges to zero.

- For increasing values of $n$ and $p_{0}$, the absolute difference between $\mathrm{ARL}_{0}, \mathrm{SDRL}_{0}$ tends to decrease.

\subsection{Required number of Phase I samples allowing similar $\mathrm{ARL}_{0}$ in both cases}

As the values of $\mathrm{ARL}_{0}$ of the hypergeometric $n p$ chart differ in the known and in the estimated parameter case, an interesting question arises how large the number $m$ of Phase I samples should be in order to have approximately the same $\mathrm{ARL}_{0}$ values in both the known and estimated parameter cases, given the same chart parameter $K=3$. In Table 11 computed values of $m$ for $N \in\{100,200,500,1000,2000,5000,10000\}$, $n \in\{25,50,75,100\}, p_{0} \in\{0.01,0.02,0.05,0.1,0.15,0.2\}$ are presented, satisfying

$$
\Delta=\frac{\left|\mathrm{ARL}_{0, m}-\mathrm{ARL}_{0, \infty}\right|}{\mathrm{ARL}_{0, \infty}} \leq 0.05 .
$$

The computation of $m$ was provided using the approximation of $X=\sum_{i=1}^{m} X_{i}$ via $Z \sim \operatorname{HYP}\left(m N, m n, p_{0}\right)$ discussed above and in Section 3.3. Depending on the values of $N, n$ and $p_{0}$, the value of $m$, complying with the constraint on the relative difference between the in-control $\mathrm{ARL}_{0, m}$ (estimated parameter case) and the in-control $\mathrm{ARL}_{0, \infty}$ (known parameter case), varies between 3 and 8060 and, in some cases, can be larger than 10000. The exceptional cases where we have non-existent values stem from combinations of small values of $N$ and $p_{0}$ mentioned above.

In particular, for $n \leq 50(n \geq 75)$ and increasing $N$ there is a positive (no) trend regarding $m$. Further, there is no tendency with respect to $m$ for increasing $n$ or $p_{0}$. As for a comparison with the binomial case (see Castagliola \& Wu [3]), the number of cases with $m$ larger than 10000 can be a bit larger $(+1)$ for $n=75$ or a bit smaller $(1-3)$ for other values of $n$ with respect to the binomial value. In addition, the results of the approximate hypergeometric case converge to the respective results of the binomial case for $N=10000$. 


\subsection{Alternative chart parameters allowing similar $\mathrm{ARL}_{0}$ in both cases}

Since there are (as in the binomial case) several cases where $m>10000$, we propose to compute alternative chart parameters $K^{\prime}$ which take the value of $m$ into account and simultaneously allow the value of $\mathrm{ARL}_{0}$ of the estimated parameter case to be as close as possible to the value of $\mathrm{ARL}_{0}$ of the known parameter case using the above mentioned approximation. These values are presented in Tables 12 18 for $N \in\{100,200,500,1000,2000,5000,10000\}, n \in\{25,50,75,100\}, p_{0} \in\{0.01,0.02,0.05,0.10,0.15,0.20\}$ and $m \in\{10,20,50,100,200\}$. For example, if $N=1000, n=50, p_{0}=0.05$ and $m=10$ (see Table 15), the alternative chart parameter is given by $K^{\prime}=2.87$ and, in this case, $\mathrm{ARL}_{0}=420.6\left(\mathrm{SDRL}_{0}=2109.6\right)$. Referring to Table 5 . $\mathrm{ARL}_{0}$ corresponding to $m \rightarrow \infty$ (i.e., the known parameter case) is $\mathrm{ARL}_{0}=424.1$. It is worth noting that the use of the new constant $K^{\prime}=2.87$ (instead of $K=3$ ) allows to reduce $\mathrm{ARL}_{0}$ from $\mathrm{ARL}_{0}=586.6$ to $\mathrm{ARL}_{0}=420.6$ (close to 424.1 ) but it also allows to reduce $\mathrm{SDRL}_{0}$ from $\mathrm{SDRL}_{0}=3088.8$ to $\mathrm{SDRL}_{0}=2109.6$. In turn, there are some cases of non-existent values due to combinations of small values of $N$ and $p_{0}$.

\subsection{Out-of-control performance of the hypergeometric $n p$ chart}

In Tables 19 25, the out-of-control ARL and SDRL values (i.e., $\mathrm{ARL}_{1}$ and $\mathrm{SDRL}_{1}$ ) are presented for the hypergeometric $n p$ chart, given $K=3$ (see triples $\left(K, \mathrm{ARL}_{1}, \mathrm{SDRL}_{1}\right)$ in the first line of each cell). These out-of-control values are calculated using the approximation of $X=\sum_{i=1}^{m} X_{i}$ via $Z \sim \operatorname{HYP}\left(m N, m n, p_{0}\right)$ discussed in Section 3.3 for $m \in\{10,20,50,100,200,1000\}$ (estimated parameter case) under the condition of reasonable control limits (see Remark 2.1). As for $m \rightarrow \infty$ (known parameter case), the out-of-control values are obtained by using exact hypergeometric distribution (see Section 2.1).

In addition, we have verified the obtained results in Tables 1925 by conducting a simulation study. In particular, for selected combinations of $\left(m, N, n, p_{0}, \tau, K=3\right)$, we have computed $\mathrm{ARL}_{1}^{\text {sim }}$ in the estimated parameter case using the same procedure as for simulation of $\mathrm{ARL}_{0}$ in Section 4.1 but with an adjustment of $\tau$, i.e., $\tau>1$ instead of $\tau=1$. Selected results of the simulation study are shown in Table 27. Comparing the exact and simulated results leads to the same conclusions as discussed in Section 4.1 .

The results of the out-of-control performance analysis of the hypergeometric $n p$ chart are given in (1)-(3) below. Moreover, we give the chart parameters $K^{\prime \prime}$, which allow the binomial $n p$ chart to obtain an $\mathrm{ARL}_{0}$ as close as possible to the $\mathrm{ARL}_{0}$ in the hypergeometric case, and present respective $\mathrm{ARL}_{1}, \mathrm{SDRL}_{1}$ (see triples $\left(K^{\prime \prime}, \mathrm{ARL}_{1}, \mathrm{SDRL}_{1}\right)$ in the second line of each cell). Comparing the triples $\left(K, \mathrm{ARL}_{1}, \mathrm{SDRL}_{1}\right)$ with $\left(K^{\prime \prime}, \mathrm{ARL}_{1}, \mathrm{SDRL}_{1}\right)$ leads to the results presented in (4)-(5) below.

Similar to the in-control performance analysis, we consider the following parameter values:

$$
\begin{aligned}
N & \in\{100,200,500,1000,2000,5000,10000\} & & \text { (see Tables 19 25) } \\
n & \in\{25,50\} & & \text { (see blocks in Tables 19 25) } \\
m & \in\{10,20,50,100,200,1000, \infty\} & & \text { (see columns in Tables 19 25) } \\
p_{0} & \in\{0.01,0.05,0.10,0.20\} & & \text { (see areas within blocks in Tables 19 25) } \\
\tau & \in\{1.1,1.2,1.5,2.0\} & & \text { (see rows in Tables 19 25) }
\end{aligned}
$$

excluding $n=75,100$ and $p_{0}=0.02,0.15$, in order to keep the tables manageable and to focus the outof-control analysis on the most common parameter values. The out-of-control fraction nonconforming $p_{1}$ is calculated via multiple $\tau, \tau>1$, of the in-control fraction nonconforming $p_{0}$, i.e., $p_{1}=\tau p_{0}$. Thus, we consider uniform proportional shifts in the process fraction nonconforming by means of $\tau=\frac{p_{1}}{p_{0}}$. Since the detection of a significant deterioration of the process quality is crucial, we analyze values of $\tau$ that represent realistic upward shifts in the process fraction nonconforming $(10 \%, 20 \%, 50 \%, 100 \%)$. 
It should be noted that there are combinations of very low values of $N, p_{0}$ and $\tau$, where the population size is too low for identifying very small shifts in the process quality level. This applies for the following combinations: $N=100, p_{0}=0.01, \tau \leq 1.5 ; N=200, p_{0}=0.01, \tau \leq 1.2 ; N=500, p_{0}=0.01, \tau=1.1$; $N=100, p_{0}=0.05, \tau=1.1$. In these cases, $\mathrm{ARL}_{0}, \mathrm{SDRL}_{0}$ instead of $\mathrm{ARL}_{1}, \mathrm{SDRL}_{1}$ are computed, which is why these values are not given in Tables 19 21. Consequently, these cases, as well as the cases where $\mathrm{ARL}_{0} \rightarrow \infty$ (see Tables 2 3), are excluded from the out-of-control analysis.

The following results are obtained from the analysis of Tables 1925

(1) Estimated parameter case (finite values of $m$ ):

- An increase in $N$ in combination with small values of $p_{0}\left(p_{0}=0.01,0.05\right)$ generally leads to a decrease of $\mathrm{ARL}_{1}$ and $\mathrm{SDRL}_{1}$. In contrast, an increase in $N$ in combination with larger values of $p_{0}\left(p_{0}=0.10,0.20\right)$ mostly results in an increase of $\mathrm{ARL}_{1}$ and $\mathrm{SDRL}_{1}$ for $n=25$ and in an increase or a decrease for $n=50$.

- An increase in $n$ provides as a tendency a decrease of $\mathrm{ARL}_{1}$ and $\mathrm{SDRL}_{1}$. This behavior is the more distinct, the larger $p_{0}, \tau$ and the lower $m$, i.e., the number of exceptions (that is, increasing values of $\mathrm{ARL}_{1}, \mathrm{SDRL}_{1}$ ) increases for lower values of $p_{0}$ in combination with lower values of $\tau$ and larger values of $m\left(p_{0}=0.01,0.05, \tau=1.1,1.2, m \geq 100\right)$.

- An increase in $m$ generally leads to a decrease of $\mathrm{ARL}_{1}$ and $\mathrm{SDRL}_{1}$, but with exceptions for $N \leq 200$ in combination with $n=25$, where an increase of $\mathrm{ARL}_{1}$ and $\mathrm{SDRL}_{1}$ is also possible.

- An increase in $p_{0}$ mostly implies a decrease of $\mathrm{ARL}_{1}, \mathrm{SDRL}_{1}$ for $p_{0} \geq 0.05$ and $\tau \geq 1.2$. Considering $\tau=1.1$ especially in combination with smaller values of $p_{0}\left(p_{0} \leq 0.05\right)$, there is mainly an increase of $\mathrm{ARL}_{1}$ and a decrease of $\mathrm{SDRL}_{1}$.

- An increase in $\tau$ provides a decrease of both $\mathrm{ARL}_{1}$ and $\mathrm{SDRL}_{1}$.

(2) Known parameter case $(m \rightarrow \infty)$ : The conclusions are quite similar as the ones obtained in (1). In general, the following applies:

- The larger $N$, the lower $\mathrm{ARL}_{1}, \mathrm{SDRL}_{1}$, except for some cases with $n=50$ and $N \leq 500$, where $\mathrm{ARL}_{1}$ and $\mathrm{SDRL}_{1}$ increase with an increasing value of $N$.

- The larger $n$, the larger $\mathrm{ARL}_{1}, \mathrm{SDRL}_{1}$, except for cases with $n=50$ and $N \leq 500$, where $\mathrm{ARL}_{1}$ and $\mathrm{SDRL}_{1}$ decrease with an increasing value of $n$.

- An increase in $p_{0}$ mostly implies a decrease of $\mathrm{ARL}_{1}, \mathrm{SDRL}_{1}$ with some contrary exceptions for $N \leq 500$.

- The larger $\tau$, the lower $\mathrm{ARL}_{1}, \mathrm{SDRL}_{1}$.

(3) Comparison of the estimated and known parameter cases:

- The values of $\mathrm{ARL}_{1}$ and $\mathrm{SDRL}_{1}$ differ in general in the known and in the estimated parameter case. In particular, for $N \geq 500$ in combination with $n=25, \mathrm{ARL}_{1}, \mathrm{SDRL}_{1}$ are larger in the estimated parameter case compared to the known parameter case, while for $N \leq 200$ they can be either larger or smaller compared to the known parameter case. For $n=50$, there is no clear tendency for positive or negative differences, except for $p_{0}=0.01$ in combination with $N \geq 500$, where $\mathrm{ARL}_{1}, \mathrm{SDRL}_{1}$ in the known parameter case are smaller than in the estimated parameter case.

- When $N, n, m, p_{0}$ or $\tau$ increases, the absolute difference in terms of $\mathrm{ARL}_{1}$ (between the known and estimated parameter case) tends to decrease but not always in a monotonic way.

(4) Comparison of the hypergeometric and binomial $n p$ charts (estimated parameter case): 
- The values of $\mathrm{ARL}_{1}$ and $\mathrm{SDRL}_{1}$ are mostly lower (or equal) for the hypergeometric $n p$ chart compared to the binomial $n p$ chart for $N \leq 5000$. Considering $N=10000$, this behavior changes slightly, i.e., the values of $\mathrm{ARL}_{1}$ and $\mathrm{SDRL}_{1}$ differ for both charts but with the same tendency.

- As a consequence of an increase in $N$, the difference between $\mathrm{ARL}_{1}, \mathrm{SDRL}_{1}$ of both charts decreases and converges to zero.

- There is no clear tendency regarding the difference between $\mathrm{ARL}_{1}, \mathrm{SDRL}_{1}$ of both charts for different values of $n, m, p_{0}, \tau$.

- In general, the value of $K^{\prime \prime}$ is the closer to 3 , the larger the value of $N$, regardless of $n, m, p_{0}$ (there is no impact of $\tau$ on $K^{\prime \prime}$ ). There are only a few exceptions for $N=100,200$, where $K^{\prime \prime}<3$ instead of $K^{\prime \prime} \geq 3$ (with a maximum positive deviation of +1.25 for $N=100$ ). That is, considering smaller values of $N, K^{\prime \prime}$ is often biased upwards in the binomial case. While larger values of $p_{0}\left(p_{0}=0.1,0.2\right)$ rather lead to values of $K^{\prime \prime}$ close to $K=3$ compared to smaller values of $p_{0}$ $\left(p_{0}=0.01,0.05\right)$, there is no clear pattern regarding the effect of an increasing value of $m$ and $n$ on $K^{\prime \prime}$.

(5) Comparison of the hypergeometric and binomial $n p$ charts (known parameter case):

- Since $K^{\prime \prime}$ is mostly equal to 3 in the known parameter case for the binomial $n p$ chart, we refer to the respective interpretations regarding Table 26 in Section 4.5. It should be noted that there are some values of $K^{\prime \prime}$ that are different from 3 , but they do not change the interpretation results.

\subsection{Out-of-control performance of the binomial $n p$ chart}

As for the out-of-control performance of the binomial $n p$ chart, the respective $\mathrm{ARL}_{1}$ and $\mathrm{SDRL}_{1}$ values are provided for $K=3$ in Table 26, and we get the results summarized in (1)-(3) below. Afterward we compare the values of Table 26 with the respective values for the hypergeometric $n p$ chart (given in the first row of each cell of Tables 19 25), and obtain results summarized in (4)-(5) below. It should be noted that in the cases where we refer to the effect observed for the hypergeometric $n p$ chart, interpretations concerning $N$ are by implication not applicable.

(1) Estimated parameter case (finite values of $m$ ):

- The impact of $n, p_{0}, \tau$ on $\mathrm{ARL}_{1}, \mathrm{SDRL}_{1}$ is the same as in the hypergeometric case.

- The general impact of $m$ on $\mathrm{ARL}_{1}, \mathrm{SDRL}_{1}$ is in turn the same as in the hypergeometric case, but with exceptions for $m \geq 200$ in combination with $p_{0}=0.05,0.10$, which provide an increase of $\mathrm{ARL}_{1}$ and mostly an increase of $\mathrm{SDRL}_{1}$.

(2) Known parameter case $(m \rightarrow \infty)$ :

- Contrary to the hypergeometric case, an increasing $n$ leads to larger values of $\mathrm{ARL}_{1}, \mathrm{SDRL}_{1}$ for $p_{0} \leq 0.05$ and to lower values of $\mathrm{ARL}_{1}, \mathrm{SDRL}_{1}$ for $p_{0} \geq 0.1$.

- There is no clear effect of a change in $p_{0}$. In particular, when $p_{0}$ increases, $\mathrm{ARL}_{1}$ and $\mathrm{SDRL}_{1}$ can increase or decrease.

- The impact of $\tau$ on $\mathrm{ARL}_{1}, \mathrm{SDRL}_{1}$ is the same as in the hypergeometric case.

(3) Comparison of the estimated and known parameter cases:

- The values of $\mathrm{ARL}_{1}$ and $\mathrm{SDRL}_{1}$ differ in general in the known and in the estimated parameter case, i.e., there is no clear tendency for positive or negative differences.

- When $n, m, p_{0}, \tau$ increases, the absolute difference in terms of $\mathrm{ARL}_{1}$ (between the known and estimated parameter case) tends to decrease but not always in a monotonic way. 
(4) Comparison of the hypergeometric and binomial $n p$ charts (estimated parameter case):

- The values of $\mathrm{ARL}_{1}$ and $\mathrm{SDRL}_{1}$ differ for both $n p$ charts. In particular, for $p_{0}=0.01,0.05$ in combination with $\tau=1.1,1.2$ and for $p_{0}=0.1,0.2$ in combination with $\tau=1.5,2.0$, there are larger and lower values of $\mathrm{ARL}_{1}, \mathrm{SDRL}_{1}$ for the hypergeometric $n p$ chart compared to the binomial np chart, respectively.

- As a consequence of an increase in $N$, the absolute difference between $\mathrm{ARL}_{1}, \mathrm{SDRL}_{1}$ for both charts decreases and converges to zero.

- When $m$ increases, the absolute difference between $\mathrm{ARL}_{1}, \mathrm{SDRL}_{1}$ for both charts decreases. There is on trend the same behavior for an increasing value of $n, p_{0}, \tau$.

(5) Comparison of the hypergeometric and binomial $n p$ charts (known parameter case):

- The values of $\mathrm{ARL}_{1}$ and $\mathrm{SDRL}_{1}$ are mostly lower for $N \leq 500$ and larger for $N \geq 1000$ in the hypergeometric case compared to the binomial case.

- For an increasing value of $N$, the absolute difference between $\mathrm{ARL}_{1}, \mathrm{SDRL}_{1}$ for both charts decreases and converges to zero. The same holds for an increasing value of $\tau$.

- For an increasing value of $n$ and a decreasing value of $p_{0}$, the absolute difference between $\mathrm{ARL}_{1}, \mathrm{SDRL}_{1}$ for both charts decreases.

\subsection{Summary of numerical analysis}

In the following, we present summarized results for the in-control and out-of-control performance analysis:

\section{Section 4.1}

1. Given unknown parameter $p_{0}$, the hypergeometric $n p$ chart with estimated parameter is preferable to the hypergeometric $n p$ chart with known parameter, since the values of $\mathrm{ARL}_{0}, \mathrm{SDRL}_{0}$ differ in general in both cases, and the absolute difference in terms of $\mathrm{ARL}_{0}$ generally decreases when $N, n, m$ or $p_{0}$ increases.

2. The exact $\mathrm{ARL}_{0}, \mathrm{SDRL}_{0}$ are throughout approximately equal to the respective approximate $\mathrm{ARL}_{0}, \mathrm{SDRL}_{0}$ with negligible relative deviations. As the absolute difference between exact and approximate $\mathrm{ARL}_{0}, \mathrm{SDRL}_{0}$ converges to zero when $N, n, m, p_{0}$ increases, the new approximation is preferable for calculations due to considerable reductions of computational time, in particular for larger parameter values.

3. The lower $N, m, p_{0}$, the more preferable the hypergeometric $n p$ chart compared to its binomial counterpart, in the known and estimated parameter case. This result is due to the following two facts:

- Considering the hypergeometric $n p$ chart, larger values of $N, m$ and/or lower values of $n$ mostly lead to a decrease of $\mathrm{ARL}_{0}$ in the estimated parameter case. The same holds for the known parameter case (except for $m$ by implication).

- $\mathrm{ARL}_{0}$ is in general larger for the hypergeometric $n p$ chart compared to the binomial $n p$ chart in both cases, and there is the tendency of a decreasing difference in terms of $\mathrm{ARL}_{0}$ when $N, m, p_{0}$ increases (estimated parameter case) and when $N$ increases or $n, p_{0}$ decreases (known parameter case).

\section{Section 4.2}

The value of $m$, which is needed to obtain run length properties as in the known parameter case for the hypergeometric $n p$ chart with estimated parameter $p_{0}$ and $3 \sigma$ control limits (i.e., $K=3$ ), can strongly vary for several combinations of $N, n, p_{0}$. This result is similar to the binomial case. 


\section{Section 4.3}

The use of an alternative chart parameter $K^{\prime}$ (especially dedicated to the number $m$ of Phase I samples) allows $\mathrm{ARL}_{0}$ corresponding to the estimated parameter case to be as close as possible to $\mathrm{ARL}_{0}$ corresponding to the known parameter case. These new chart parameters also enable to reduce $\mathrm{SDRL}_{0}$ of the hypergeometric $n p$ chart in the estimated parameter case.

\section{Sections 4.4 and 4.5 ,}

1. Given unknown parameter $p_{0}$, the hypergeometric $n p$ chart with estimated parameter is preferable to the hypergeometric $n p$ chart with known parameter, since the values of $\mathrm{ARL}_{1}, \mathrm{SDRL}_{1}$ differ in general in both cases and the absolute difference in terms of $\mathrm{ARL}_{1}$ tends to decrease for increasing values of $N, n, m$ or $\tau$.

2. The hypergeometric $n p$ chart is preferable compared to its binomial counterpart in situations with lower $N$ in combination with lower $n, m, p_{0}, \tau$. This result is due to the following two facts:

- Given $K=3$, the values of $\mathrm{ARL}_{1}, \mathrm{SDRL}_{1}$ differ for both $n p$ charts. That is, the magnitude of $\mathrm{ARL}_{1}, \mathrm{SDRL}_{1}$ can be biased up- or downwards in the binomial case. However, when $N, n, m, \tau$ increases, the absolute difference in terms of $\mathrm{ARL}_{1}, \mathrm{SDRL}_{1}$ between both charts decreases, in the estimated and known parameter case.

- Given similar values of $\mathrm{ARL}_{0}$, the corresponding values of $\mathrm{ARL}_{1}, \mathrm{SDRL}_{1}$ of the hypergeometric $n p$ chart are mostly lower compared to the binomial $n p$ chart for smaller values of $N$.

\section{$5 \quad$ Illustrative example}

A local company uses an automatic welding machine to weld two pieces of stainless steel together. This machine uses a MIG welding technology ("Metal Inert Gas" using electricity to melt and join pieces of metal together). For every pair of stainless steel pieces, the welding process takes about 30s. Therefore, a batch of $N=1000$ pieces is obtained everyday after a continuous production of about 8 hours. In order to evaluate the quality of the welds, $n=50$ pieces are taken randomly from the batch, without replacement, and a destructive weld testing (a transverse tension test) is operated to evaluate their tensile properties. The results are the number of pieces for which the welds did not pass the destructive test (and are therefore considered as nonconforming ones) during $m=10$ consecutive days (2 working weeks) considered by the company as an in-control Phase I period of production (see values $x_{i}, i=1, \ldots, 10$, in Table 1).

Table 1: Number of nonconforming pieces during Phase I and Phase II

\begin{tabular}{c|cccccccccc}
$i$ & 1 & 2 & 3 & 4 & 5 & 6 & 7 & 8 & 9 & 10 \\
\hline$x_{i}$ & 4 & 1 & 2 & 1 & 3 & 3 & 3 & 2 & 2 & 4 \\
$y_{i}$ & 3 & 3 & 2 & 2 & 3 & 7 & 1 & 3 & 4 & 2
\end{tabular}

From these results, we can estimate the in-control proportion of nonconforming welds as $\hat{p}_{0}=0.05$. If we assume a hypergeometric $n p$ chart with known parameter $p_{0}=0.05$ and $K=3$, the corresponding control limits can be obtained using (2.1) and (2.2), and they are equal to

$$
\mathrm{LCL}_{\mathrm{HYP}}=\max \{0,\lceil-2.0085\rceil\}=0 \quad \mathrm{UCL}_{\mathrm{HYP}}=\lfloor 7.0085\rfloor=7
$$

For these values, the corresponding in-control $\mathrm{ARL}$ is $\mathrm{ARL}_{0}=424.0830$. Now, if we assume a hypergeometric $n p$ chart with estimated parameter $\hat{p}_{0}=0.05$ based on $m=10$ Phase I samples, the corrected chart constant to be used is found to be $K^{\prime}=2.87$ yielding the following corrected control limits (see (2.4) and 2.5)

$$
\widehat{\mathrm{LCL}}_{\mathrm{HYP}}=\max \{0,\lceil-1.8131\rceil\}=0 \quad \widehat{\mathrm{UCL}}_{\mathrm{HYP}}=\lfloor 6.8131\rfloor=6
$$


These corrected control limits allow the hypergeometric $n p$ chart with estimated parameter to have an exact in-control $\mathrm{ARL}_{0}=421.0615$ to be close to the one obtained in the known parameter case, i.e., $\mathrm{ARL}_{0}=424.0830$.

The values $y_{i}, i=1, \ldots, 10$, in Table 1 are the number of pieces for which the welds of the pieces did not pass the destructive test during a Phase II period of $k=10$ days of production.
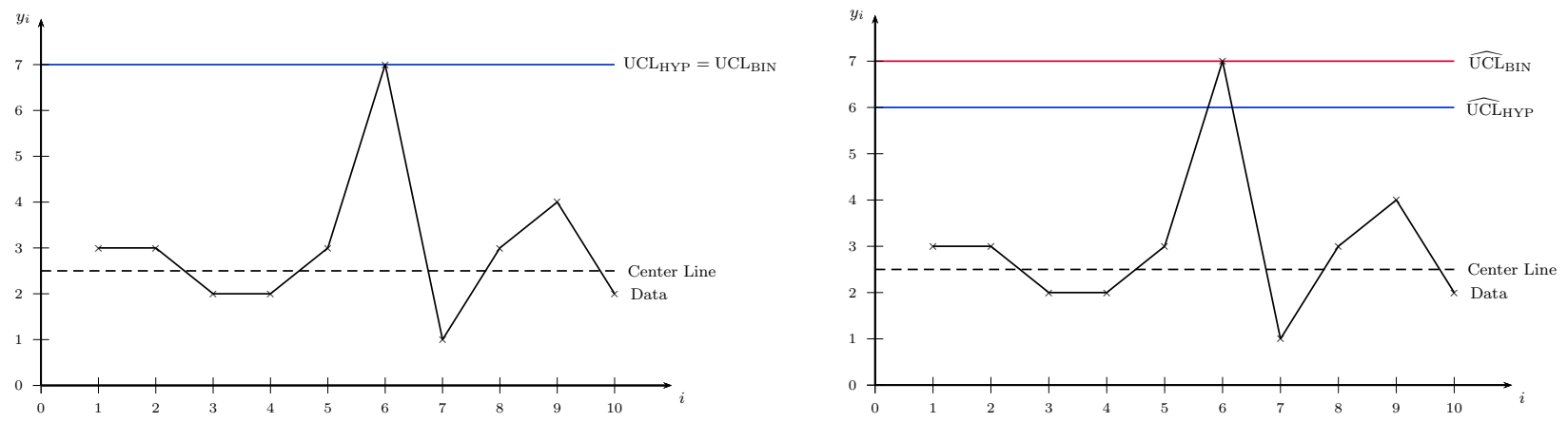

Figure 2: The hypergeometric $n p$ chart with known parameter (left) and estimated parameter (right)

The corresponding hypergeometric np chart with known and estimated parameter is plotted in Figure 2 along with control limits (5.1) and corrected control limits (5.2), respectively. Note that numerical results are displayed by a line plot instead of a scatter plot for better output illustration. As it can be seen, up to the 5th day in Phase II, the welding process seems to be in-control as all the points are within the control limits. During the 6th day, something clearly happened and a higher percentage of pieces did not pass the destructive test. Therefore, the process is declared as out-of-control in the case of estimated parameter $\left(y_{6}>\widehat{\mathrm{UCL}}_{\mathrm{HYP}}\right)$, while the hypergeometric $n p$ chart with known parameter incorrectly triggers no out-ofcontrol signal $\left(y_{6} \ngtr \mathrm{UCL}_{\mathrm{HYP}}\right)$. A search for potential assignable causes impacting the welding process has shown that an improper electrical resistance due to the connection between the power source and the gun's power cable plug occurred too frequently leading to a poor and inconsistent weld quality. Once this issue has been fixed, the production went back to an in-control state as it can be seen with points corresponding to days 7-10 within the control limits.

As for a comparison with the common binomial $n p$ chart, the control limits in the known and the estimated parameter case are as follows:

$$
\begin{array}{lll}
\mathrm{LCL}_{\mathrm{BIN}}=\max \{0,\lceil-2.1233\rceil\}=0 & \mathrm{UCL}_{\mathrm{BIN}}=\lfloor 7.1233\rfloor=7 & \left(K=3, \mathrm{ARL}_{0}=313.6425\right) \\
\widehat{\mathrm{LCL}}_{\mathrm{BIN}}=\max \{0,\lceil-2.0463\rceil\}=0 & \widehat{\mathrm{UCL}}_{\mathrm{BIN}}=\lfloor 7.0463\rfloor=7 & \left(K^{\prime}=2.95, \mathrm{ARL}_{0}=406.4205\right)
\end{array}
$$

These control limits can also be found in Figure 2. Compared to the hypergeometric $n p$ chart with estimated parameter, the process is incorrectly declared as in-control during Phase II in both the known and the estimated parameter case and no signal is triggered on the 6th day. It should be noted that (as in the hypergeometric case) the corrected control limits (5.3) allow the binomial $n p$ chart to have an in-control $\mathrm{ARL}_{0}=406.4205$ to be close to the one obtained for the hypergeometric $n p$ chart in the known (as well as estimated) parameter case.

\section{Conclusions}

In this paper, we have extended the analysis of hypergeometric attribute control charts with regard to their run length properties in both the known and the estimated parameter cases. We have also compared the 
results with the respective results obtained for the binomial $n p$ chart. From these comparisons, we have gained some interesting insights that may be useful for practical applications of the hypergeometric $n p$ chart.

In the following, we briefly summarize the particular contributions provided in this paper and comment on the respective obtained results.

1. By introducing the hypergeometric $n p$ chart, we fill a gap in the framework of hypergeometric attribute control charts. This chart is more convenient for practical applications in comparison with the hypergeometric $p$ chart as it deals with integer values.

2. We extend the analysis of hypergeometric control charts for monitoring attributes data to the more realistic (and thus more important) unknown parameter case and study the effect of parameter estimation on control charts performance.

3. We compare the in-control and the out-of-control run length properties of the hypergeometric $n p$ chart in both the known and estimated parameter cases, and therefore we discuss both sides of the coin regarding the overall impact of parameter estimation on control charts performance. Guided by the obtained results, we recommend the use of the hypergeometric $n p$ chart with estimated parameter for practical applications, given the in-control proportion $p_{0}$ of nonconforming units is unknown, instead of the corresponding chart with known parameter, which provides unpredictable (i.e., up- or downwards) biased in-control and out-of-control measures.

4. We investigate the required number of Phase I samples in the framework of the hypergeometric $n p$ chart with estimated parameter $p$ in order to obtain similar values of the in-control average run length $\mathrm{ARL}_{0}$ compared to the known parameter case. However, the obtained results are not predictable and in turn confirm the recommendation above (see 3.).

5. In order to facilitate the implementation of the hypergeometric $n p$ chart with estimated parameter for the practitioner, we consider alternative chart parameters that allow the hypergeometric $n p$ chart with estimated parameter $p$ to have approximately the same values of $\mathrm{ARL}_{0}$ as the ones obtained when the parameter $p$ is known. Tables 1218 show the respective alternative chart parameters for various combinations of $N, n, m, p_{0}$ and therefore enable a direct implementation in practice.

6. We perform a comprehensive in-control and out-of-control comparison of the hypergeometric $n p$ chart with the binomial $n p$ chart, considering either the same chart parameter $K=3$ or similar values of $\mathrm{ARL}_{0}$. In this way, we also fill a gap in the literature with regard to the non-existing out-of-control analysis of the binomial $n p$ chart. Guided by the obtained results, we recommend the use of the hypergeometric $n p$ chart instead of the binomial $n p$ chart in both the known and estimated parameter cases, given sampling without replacement from a finite population. This is due to the fact that the binomial $n p$ chart in general provides downwards-biased in-control measures, and the out-of-control measures either differ for both charts, given $K=3$, or are mostly lower compared to the binomial $n p$ chart for smaller values of $N$, given similar values of $\mathrm{ARL}_{0}$. Since the absolute differences in terms of in-control and out-of-control measures decrease between both charts when $N$ increases, the above recommendation is of particular importance for small population sizes $N$ in combination with smaller values of $n, m, p_{0}, \tau$. The same applies to the recommendation given in 3 .

To achieve the above results, we had to compute the probability distribution of $X=\sum_{i=1}^{m} X_{i}$ with i.i.d. random variables $X_{i} \sim \operatorname{HYP}\left(N, n, p_{0}\right), i=1, \ldots, m$. However, the computational effort using convolution with double-precision floating point numbers is very extensive, especially for combinations of larger values of $N, n, m, p_{0}$. In order to get a feasible solution that leads to a considerably faster calculation and simultaneously ensures a high accuracy of the results, we have proposed a new approximation for the probability distribution of $X$. Although $X$ is in general not hypergeometric-distributed, we have shown by extensive computations and numerical analyzes that $X$ can well be approximated by a random variable 
$Z \sim \operatorname{HYP}\left(m N, m n, p_{0}\right)$. By using this approximation the time required for the computation of $F_{X}$ and $f_{X}$ can be reduced to a few seconds while keeping a remarkable high accuracy with only negligible deviations compared to the exact distribution obtained via convolution. This result has been considered for the first time in the literature and may be helpful for numerous applications that go beyond the hypergeometric $n p$ chart, for instance, it can be used for risk aggregation in quantitative risk management (e.g. credit, insurance, market, and operational risks, see Klugman et al. [26]).

\section{Acknowledgments:}

The authors would like to thank three anonymous reviewers for their valuable feedback and suggestions, which were very important and helpful to significantly improve the paper. The authors are also grateful to the student assistant Marcel Gaweda for his engaged support. Finally, the authors gratefully acknowledge the generous extension of the submission deadline granted by Professor Steffen Rebennack.

\section{References}

[1] Braun, W. J. (1999): Run length distributions for estimated attributes charts. Metrika, 50, pp. 121-129.

[2] Castagliola, P. \& P. E. Maravelakis (2011): A CUSUM control chart for monitoring the variance when parameters are estimated. Journal of Statistical Planning and Inference, 141(4), pp. 1463-1478.

[3] Castagliola, P. \& S. Wu (2012): Design of the $c$ and $n p$ charts when the parameters are estimated. International Journal of Reliability, Quality and Safety Engineering, 19(2), 1250010, pp. 1-16.

[4] Castagliola, P., G. Celano \& G. Chen (2009): The exact run length distribution and design of the $S^{2}$ chart when the in-control variance is estimated. International Journal of Reliability, Quality and Safety Engineering, 16(1), pp. 23-38.

[5] Castagliola, P., P. C. Oprime \& M. B. C. Khoo (2017): The Double Sampling $S^{2}$ Chart with Estimated Process Variance. Communications in Statistics - Theory and Methods, 46(7), pp. 3556-3573.

[6] Celano, G. \& S. Chakraborti (2020): A distribution-free Shewhart-type Mann-Whitney control chart for monitoring finite horizon productions. International Journal of Production Research, in press.

[7] Chakraborti, S. (2006): Parameter estimation and design considerations in prospective applications of the $\bar{X}$ chart. Journal of Applied Statistics, 33(4), pp. 439-459.

[8] Chakraborti, S. \& S. W. Human (2006): Parameter estimation and performance of the $p$-chart for attributes data. IEEE Transactions on Reliability, 55(3), pp. 559-566.

[9] Chakraborti, S. \& S. W. Human (2008): Properties and performance of the $c$-chart for attributes data. Journal of Applied Statistics, 35(1), pp. 89-100.

[10] Chong, Z. L., M. B. C. Khoo, W. L. Teoh, H. W. You \& P. Castagliola (2019): Optimal design of side sensitive modified group runs (SSMGR) $\bar{X}$ chart when process parameters are estimated. Quality and Reliability Engineering International, 35(1), pp. 246-262.

[11] Chong, Z. L., M. B. C. Khoo, W. L. Teoh, W. C. Yeong \& S. L. Lim (2020): Optimal design of the modified group runs (MGR) $\bar{X}$ chart when process parameters are estimated. Communications in Statistics - Simulation and Computation, 49(1), pp. 244-260.

[12] Chong, Z. L., K. L. Tan, M. B. C. Khoo, W. L. Teoh \& P. Castagliola (2020): Optimal designs of the exponentially weighted moving average (EWMA) median chart for known and estimated parameters based on median run length. Communications in Statistics - Simulation and Computation, in press.

[13] Chukhrova, N. \& A. Johannssen (2019): Improved control charts for fraction non-conforming based on hypergeometric distribution. Computers \& Industrial Engineering, 128, pp. 795-806.

[14] Chukhrova, N. \& A. Johannssen (2019): Hypergeometric p-chart with dynamic probability control limits for monitoring processes with variable sample and population sizes. Computers 83 Industrial Engineering, 136, pp. 681-701.

[15] De Pril, N. (1985): Recursions for convolutions of arithmetic distributions. ASTIN Bulletin, 15(2), pp. $135-139$. 
[16] Dickson, D. C. M. (2016): Insurance Risk and Ruin. 2nd edition, Cambridge University Press.

[17] Faraz, A., C. Heuchenne \& E. Saniga (2017): The $n p$ Chart with Guaranteed In-control Average Run Lengths. Quality and Reliability Engineering International, 33(5), pp. 1057-1066.

[18] Filho, D. M. \& M. Valk (2020): Dynamic VAR model-based control charts for batch process monitoring. European Journal of Operational Research, 285(1), pp. 296-305.

[19] Hashemian, S. M., R. Noorossana, A. Keyvandarian \& M. A. Shekary (2016): Performance of adaptive $n p$-chart with estimated parameter. International Journal of Quality \& Reliability Management, 33(6), pp. 769-791.

[20] Hu, X. L. \& P. Castagliola (2019): A Re-Evaluation of the Run Rules $\bar{X}$ Chart when the Process Parameters are Unknown. Quality Technology \& Quantitative Management, 16(6), pp. 696-725.

[21] Hu, X. L., P. Castagliola, Y. Z. Ma \& W. D. Huang (2018): Guaranteed in-control performance of the Synthetic $\bar{X}$ Chart with Estimated Parameters. Quality and Reliability Engineering International, 34(5), pp. 759-771.

[22] Hu, X. L., P. Castagliola, X. J. Zhou \& A. A. Tang (2019): Conditional design of the EWMA median chart with estimated parameters. Communications in Statistics - Theory and Methods, 48(8), pp. 1871-1889.

[23] Jensen, W. A., L. A. Jones-Farmer, C. W. Champ \& W. H. Woodall (2006): Effects of parameters estimation on control chart properties: A literature review. Journal of Quality Technology, 38(4), pp. 349-364.

[24] Jones, L. A., C. W. Champ \& S. E. Rigdon (2001): The performance of exponentially weighted moving average charts with estimated parameters. Technometrics, 43(2), pp. 156-167.

[25] Khoo, M. B. C., S. Saha, H. C. Lee \& P. Castagliola (2019): Variable Sampling Interval Exponentially Weighted Moving Average Median Chart with Estimated Process Parameters. Quality and Reliability Engineering International, 35(8), pp. 2732-2748.

[26] Klugman, S. A., H. H. Panjer \& G. E. Willmot (2012): Loss Models: From Data to Decisions. 4th edition, Wiley Series in Probability and Statistics.

[27] Lee, M. H. \& M. B. C. Khoo (2019): Double sampling $n p$ chart with estimated process parameter. Communications in Statistics - Simulation and Computation, in press.

[28] Lee, M. H., M. B. C. Khoo, X. Chew \& P. H. H. Then (2020): Economic-statistical design of synthetic $n p$ chart with estimated process parameter. PLOS ONE, 15(4), e0230994.

[29] Maravelakis, P. E. \& P. Castagliola (2009): An EWMA chart for monitoring the process standard deviation when parameters are estimated. Computational Statistics and Data Analysis, 53(7), pp. 2653-2664.

[30] Maravelakis, P. E., J. Panaretos \& S. Psarakis (2002): Effect of estimation of the process parameters on the control limits of the univariate control charts for process dispersion. Communications in Statistics - Simulation and Computation, 31(3), pp. 443-461.

[31] Mitra, A., L. Bok \& S. Chakraborti (2019): An adaptive exponentially weighted moving average-type control chart to monitor the process mean. European Journal of Operational Research, 279(3), pp. 902-911.

[32] Motsepa, C., J.-C. Malela-Majika, P. Castagliola \& S. C. Shongwe (2020): A Side-Sensitive Double Sampling $\bar{X}$ Monitoring Scheme with Estimated Process Parameters. Communications in Statistics Simulation and Computation, in press.

[33] Nedumaran, G. \& J. J. Pignatiello Jr. (2001): On estimating $\bar{X}$ control chart limits. Journal of Quality Technology, 33(2), pp. 206-212.

[34] Niewiadomska-Bugaj, M. \& R. Bartoszynski (2020): Probability and Statistical Inference. 3rd edition, Wiley-Interscience.

[35] Nguyen, H. D., K. P. Tran \& K. D. Tran (2020): The effect of measurement errors on the performance of the Exponentially Weighted Moving Average control charts for the Ratio of Two Normally Distributed Variables. European Journal of Operational Research, in press. 
[36] Psarakis, S., A. K. Vyniou \& P. Castagliola (2014): Some recent developments on the effects of parameter estimation on control charts. Quality and Reliability Engineering International, 30(8), pp. $1113-1129$.

[37] Song, Z., A. Mukherjee, Y. Liu \& J. Zhang (2019): Optimizing joint location-scale monitoring - An adaptive distribution-free approach with minimal loss of information. European Journal of Operational Research, 274(3), pp. 1019-1036.

[38] Song, Z., A. Mukherjee \& J. Zhang (2021): Some robust approaches based on copula for monitoring bivariate processes and component-wise assessment. European Journal of Operational Research, 289(1), pp. $177-196$.

[39] Tang, A. A., P. Castagliola, X. Hu \& J. Sun (2019): The adaptive EWMA median chart for known and estimated parameters. Journal of Statistical Computation and Simulation, 89(5), pp. 844-863.

[40] Teoh, W. L., M. B. C. Khoo, P. Castagliola, W. C. Yeong \& S. Y. Teh (2017): Run-sum control charts for monitoring the coefficient of variation. European Journal of Operational Research, 257(1), pp. $144-158$.

[41] Tiplica, T. (2015): $\mathrm{ARL}_{1}$ of the Attribute $c$ Control Chart with Estimated Parameter. International Journal of Reliability, Quality and Safety Engineering, 22(2), 1550009, pp. 1-24.

[42] Wu, S., P. Castagliola \& M. B. C. Khoo (2016): Run Rules based Phase II $c$ and $n p$ Charts when Process Parameters are Unknown. Communications in Statistics - Theory and Methods, 45(4), pp. 1182-1197.

[43] Zhang, Y., P. Castagliola, Z. Wu \& M. B. C. Khoo (2011): The synthetic $\bar{X}$ chart with estimated parameters. IIE Transactions, 43(9), pp. 676-687.

[44] Zwetsloot, I. M. \& W. H. Woodall (2017): A head-to-head comparative study of the conditional performance of control charts based on estimated parameters. Quality Engineering, 29(2), pp. 244-253.

\section{Appendix: Tables for numerical analysis}


Table 2: $\left(\mathrm{ARL}_{0}, \mathrm{SDRL}_{0}\right)$ for the hypergeometric $n p$ chart with $N=100, n \in\{25,50,75,100\}, p_{0} \in$ $\{0.01,0.02,0.05,0.10,0.15,0.20\}, m \in\{10,20,50,100,200,1000, \infty\}, K=3$

\begin{tabular}{|c|c|c|c|c|c|c|c|}
\hline$p_{0}$ & $m=10$ & $m=20$ & $m=50$ & $m=100$ & $m=200$ & $m=1000$ & $m \rightarrow \infty$ \\
\hline \multicolumn{8}{|c|}{$n=25$} \\
\hline 0.01 & $(\infty, \infty)$ & $(\infty, \infty)$ & $(\infty, \infty)$ & $(\infty, \infty)$ & $(\infty, \infty)$ & $(\infty, \infty)$ & $(\infty, \infty)$ \\
\hline 0.02 & $(\infty, \infty)$ & $(\infty, \infty)$ & $(\infty, \infty)$ & $(\infty, \infty)$ & $(\infty, \infty)$ & $(\infty, \infty)$ & $(\infty, \infty)$ \\
\hline 0.05 & $(729.8,1199.7)$ & $(799.3,1238.7)$ & $(898.8,1288.7)$ & $(989.0,1326.5)$ & $(1096.1,1362.2)$ & $(1332.2,1409.5)$ & $(1417.0,1416.5)$ \\
\hline 0.10 & $(2937.5,61362.8)$ & $(949.9,4647)$ & $(597.8,1355.8)$ & $(491.2,650.2)$ & $(484.8,489.5)$ & $(486.0,485.5)$ & $(486.0,485.5)$ \\
\hline 0.15 & $(1648.7,7301.5)$ & $(979.5,2902.8)$ & $(606.1,1126.2)$ & $(536.2,666.4)$ & $(532.6,538.9)$ & $(535.0,534.5)$ & $(535.0,534.5)$ \\
\hline 0.20 & $(467.7,627.7)$ & $(539.4,685.4)$ & $(604.1,732.4)$ & $(683.0,773.8)$ & $(747.9,797.2)$ & $(812.4,812.8)$ & $(813.5,813.0)$ \\
\hline \multicolumn{8}{|c|}{$n=50$} \\
\hline 0.01 & $(\infty, \infty)$ & $(\infty, \infty)$ & $(\infty, \infty)$ & $(\infty, \infty)$ & $(\infty, \infty)$ & $(\infty, \infty)$ & $(\infty, \infty)$ \\
\hline 0.02 & $(\infty, \infty)$ & $(\infty, \infty)$ & $(\infty, \infty)$ & $(\infty, \infty)$ & $(\infty, \infty)$ & $(\infty, \infty)$ & $(\infty, \infty)$ \\
\hline 0.05 & $(\infty, \infty)$ & $(\infty, \infty)$ & $(\infty, \infty)$ & $(\infty, \infty)$ & $(\infty, \infty)$ & $(\infty, \infty)$ & $(\infty, \infty)$ \\
\hline 0.10 & $(648.4,792.0)$ & $(742.2,821.8)$ & $(813.2,837.4)$ & $(837.2,841.3)$ & $(842.4,842.0)$ & $(842.6,842.1)$ & $(842.6,842.1)$ \\
\hline 0.15 & $(393.9,443.5)$ & $(405.8,436.9)$ & $(390.8,417.9)$ & $(363.2,392.8)$ & $(331.0,359.5)$ & $(273.8,282.0)$ & $(261.8,261.3)$ \\
\hline 0.20 & $(375.8,504.1)$ & $(375.0,462.8)$ & $(417.2,545.7)$ & $(448.6,607.4)$ & $(492.1,682.1)$ & $(686.1,921.5)$ & $(1236.7,1236.2)$ \\
\hline \multicolumn{8}{|c|}{$n=75$} \\
\hline 0.01 & $(\infty, \infty)$ & $(\infty, \infty)$ & $(\infty, \infty)$ & $(\infty, \infty)$ & $(\infty, \infty)$ & $(\infty, \infty)$ & $(\infty, \infty)$ \\
\hline 0.02 & $(\infty, \infty)$ & $(\infty, \infty)$ & $(\infty, \infty)$ & $(\infty, \infty)$ & $(\infty, \infty)$ & $(\infty, \infty)$ & $(\infty, \infty)$ \\
\hline 0.05 & $(910.0,1293.8)$ & $(1040.2,1344.7)$ & $(1220.1,1392.3)$ & $(1335.5,1409.9)$ & $(1399.9,1415.5)$ & $(1417.0,1416.5)$ & $(1417.0,1416.5)$ \\
\hline 0.10 & $(613.0,1423.3)$ & $(494.4,691.3)$ & $(485.4,486.6)$ & $(486.0,485.5)$ & $(486.0,485.5)$ & $(486.0,485.5)$ & $(486.0,485.5)$ \\
\hline 0.15 & $(533.6,993.5)$ & $(512.6,663.3)$ & $(531.6,543.3)$ & $(534.7,534.5)$ & $(535.0,534.5)$ & $(535.0,534.5)$ & $(535.0,534.5)$ \\
\hline 0.20 & $(423.9,569.1)$ & $(513.1,645.2)$ & $(615.3,720.0)$ & $(709.5,770.1)$ & $(778.1,799.1)$ & $(813.4,813.0)$ & $(813.5,813.0)$ \\
\hline \multicolumn{8}{|c|}{$n=100$} \\
\hline 0.01 & $(\infty, \infty)$ & $(\infty, \infty)$ & $(\infty, \infty)$ & $(\infty, \infty)$ & $(\infty, \infty)$ & $(\infty, \infty)$ & $(\infty, \infty)$ \\
\hline 0.02 & $(\infty, \infty)$ & $(\infty, \infty)$ & $(\infty, \infty)$ & $(\infty, \infty)$ & $(\infty, \infty)$ & $(\infty, \infty)$ & $(\infty, \infty)$ \\
\hline 0.05 & $(\infty, \infty)$ & $(\infty, \infty)$ & $(\infty, \infty)$ & $(\infty, \infty)$ & $(\infty, \infty)$ & $(\infty, \infty)$ & $(\infty, \infty)$ \\
\hline 0.10 & $(\infty, \infty)$ & $(\infty, \infty)$ & $(\infty, \infty)$ & $(\infty, \infty)$ & $(\infty, \infty)$ & $(\infty, \infty)$ & $(\infty, \infty)$ \\
\hline 0.15 & $(\infty, \infty)$ & $(\infty, \infty)$ & $(\infty, \infty)$ & $(\infty, \infty)$ & $(\infty, \infty)$ & $(\infty, \infty)$ & $(\infty, \infty)$ \\
\hline 0.20 & $(\infty, \infty)$ & $(\infty, \infty)$ & $(\infty, \infty)$ & $(\infty, \infty)$ & $(\infty, \infty)$ & $(\infty, \infty)$ & $(\infty, \infty)$ \\
\hline
\end{tabular}

Table 3: $\left(\mathrm{ARL}_{0}, \mathrm{SDRL}_{0}\right)$ for the hypergeometric $n p$ chart with $N=200, n \in\{25,50,75,100\}, p_{0} \in$ $\{0.01,0.02,0.05,0.10,0.15,0.20\}, m \in\{10,20,50,100,200,1000, \infty\}, K=3$

\begin{tabular}{|c|c|c|c|c|c|c|c|}
\hline$p_{0}$ & $m=10$ & $m=20$ & $m=50$ & $m=100$ & $m=200$ & $m=1000$ & $m \rightarrow \infty$ \\
\hline \multicolumn{8}{|c|}{$n=25$} \\
\hline 0.01 & $(49.9,62.8)$ & $(59.7,65.1)$ & $(65.7,65.8)$ & $(66.3,65.8)$ & $(66.3,65.8)$ & $(66.3,65.8)$ & $(66.3,65.8)$ \\
\hline 0.05 & $(1211.4,28091.1)$ & $(466.1,1831.0)$ & $(297.6,597.8)$ & $(280.0,321.4)$ & $(290.7,295.3)$ & $(296.5,296.0)$ & $(296.5,296.0)$ \\
\hline 0.10 & $(990.1,7284.9)$ & $(497.0,1341.7)$ & $(365.7,639.6)$ & $(299.1,516.0)$ & $(236.5,370.8)$ & $(189.5,189.7)$ & $(189.4,188.9)$ \\
\hline 0.15 & $(1041.2,5026.2)$ & $(601.2,1389.3)$ & $(457.4,727.6)$ & $(387.7,615.0)$ & $(314.3,487.6)$ & $(225.6,233.7)$ & $(223.3,222.8)$ \\
\hline 0.20 & $(731.5,1748.0)$ & $(603.4,1129.5)$ & $(500.5,793.5)$ & $(422.3,617.8)$ & $(359.9,441.0)$ & $(331.6,331.2)$ & $(331.6,331.1)$ \\
\hline 0.01 & $(\infty, \infty)$ & $(\infty, \infty)$ & $(\infty, \infty)$ & $(\infty, \infty)$ & $(\infty, \infty)$ & $(\infty, \infty)$ & $(\infty, \infty)$ \\
\hline 0.02 & $(234.1,273.1)$ & $(250.0,276.5)$ & $(276.4,280.0)$ & $(280.4,280.3)$ & $(280.9,280.4)$ & $(280.9,280.4)$ & $(280.9,280.4)$ \\
\hline 0.05 & $(2954.7,48065.4)$ & $(990.5,4840.4)$ & $(590.8,1349.2)$ & $(425.2,763.7)$ & $(370.7,415.7)$ & $(365.3,364.8)$ & $(365.3,364.8)$ \\
\hline 0.10 & $(700.8,1292.5)$ & $(698.7,1252.8)$ & $(565.9,945.6)$ & $(476.0,674.1)$ & $(428.9,472.9)$ & $(418.5,418.0)$ & $(418.5,418)$ \\
\hline 0.15 & $(412.8,552.2)$ & $(427.7,541.1)$ & $(429.6,530.5)$ & $(468.8,578.5)$ & $(522.0,635.5)$ & $(669.5,749.7)$ & $(807.2,806.7)$ \\
\hline 0.20 & $(376.8,504.1)$ & $(401.6,487.5)$ & $(438.9,486.1)$ & $(466.6,492.4)$ & $(491.5,501.0)$ & $(507.2,506.7)$ & $(507.2,506.7)$ \\
\hline 0.05 & $(4515.1,13609.2)$ & $(2994.1,10364.3)$ & $(1325.7,3829.8)$ & $(1190.8,1564.1)$ & $(1269.3,1345.8)$ & $(1357.6,1357.7)$ & $(1358.3,1357.8)$ \\
\hline 0.10 & $(474.8,676.7)$ & $(503.0,661.8)$ & $(482.3,594.3)$ & $(441.8,518.0)$ & $(400.8,438.1)$ & $(371.7,371.3)$ & $(371.6,371.1)$ \\
\hline 0.15 & $(352.2,410.5)$ & $(378.8,419.7)$ & $(412.4,437.1)$ & $(428.1,445.4)$ & $(440.4,453.2)$ & $(471.0,473.3)$ & $(478.0,477.5)$ \\
\hline 0.20 & $(325.6,390.1)$ & $(369.5,429.1)$ & $(423.7,481.0)$ & $(471.7,522.0)$ & $(523.3,557.4)$ & $(590.7,592.6)$ & $(594.9,594.4)$ \\
\hline \multicolumn{8}{|c|}{$n=100$} \\
\hline 0.01 & $(\infty, \infty)$ & $(\infty, \infty)$ & $(\infty, \infty)$ & $(\infty, \infty)$ & $(\infty, \infty)$ & $(\infty, \infty)$ & $(\infty, \infty)$ \\
\hline 0.02 & $(\infty, \infty)$ & $(\infty, \infty)$ & $(\infty, \infty)$ & $(\infty, \infty)$ & $(\infty, \infty)$ & $(\infty, \infty)$ & $(\infty, \infty)$ \\
\hline 0.05 & $(542.4,622.7)$ & $(588.5,635.8)$ & $(635.2,645.8)$ & $(647.2,647.8)$ & $(648.5,648.0)$ & $(648.5,648.0)$ & $(648.5,648.0)$ \\
\hline 0.10 & $(423.9,568.2)$ & $(482.0,586.8)$ & $(566.9,625.5)$ & $(613.3,639.9)$ & $(639.8,646.6)$ & $(650.2,649.7)$ & $(650.2,649.7)$ \\
\hline 0.15 & $(340.8,414.2)$ & $(384.4,440.8)$ & $(418.2,449.2)$ & $(415.7,430.5)$ & $(404.9,409.2)$ & $(398.2,397.7)$ & $(398.2,397.7)$ \\
\hline 0.20 & $(327.4,385.7)$ & $(364.9,410.1)$ & $(402.1,429.8)$ & $(419.2,433.0)$ & $(423.1,426.3)$ & $(422.8,422.3)$ & $(422.8,422.3)$ \\
\hline
\end{tabular}


Table 4: $\left(\mathrm{ARL}_{0}, \mathrm{SDRL}_{0}\right)$ for the hypergeometric $n p$ chart with $N=500, n \in\{25,50,75,100\}, p_{0} \in$ $\{0.01,0.02,0.05,0.10,0.15,0.20\}, m \in\{10,20,50,100,200,1000, \infty\}, K=3$

\begin{tabular}{|c|c|c|c|c|c|c|c|}
\hline$p_{0}$ & $m=10$ & $m=20$ & $m=50$ & $m=100$ & $m=200$ & $m=1000$ & $m \rightarrow \infty$ \\
\hline \multicolumn{8}{|c|}{$n=25$} \\
\hline 0.01 & $(851.8,35047.1)$ & $(275.7,1416.7)$ & $(159.2,457.5)$ & $(105.7,337.6)$ & $(59.0,165.8)$ & $(45.7,45.2)$ & $(45.7,45.2)$ \\
\hline 0.02 & $(575.9,8001.7)$ & $(233.7,869.8)$ & $(162.8,415.5)$ & $(110.1,213.1)$ & $(95.9,105.6)$ & $(95.0,94.5)$ & $(95.0,94.5)$ \\
\hline 0.05 & $(501.5,4202.3)$ & $(294.7,826.1)$ & $(216.1,394.5)$ & $(183.8,229.9)$ & $(179.9,182.1)$ & $(180.2,179.7)$ & $(180.2,179.7)$ \\
\hline 0.10 & $(673.6,3450.3)$ & $(405.3,992.7)$ & $(306.1,471.3)$ & $(285.6,426.6)$ & $(259.6,396.9)$ & $(173.7,261.2)$ & $(129.7,129.2)$ \\
\hline 0.15 & $(736.1,3110.3)$ & $(480.7,1063.2)$ & $(383.9,564.7)$ & $(374.2,502.9)$ & $(363.8,493.0)$ & $(338.5,470.3)$ & $(154.2,153.7)$ \\
\hline 0.20 & $(665.3,1534.0)$ & $(554.1,1122.7)$ & $(452.3,669.5)$ & $(416.5,592.8)$ & $(373.6,538.4)$ & $(257.1,326.4)$ & $(224.4,223.9)$ \\
\hline \multicolumn{8}{|c|}{$n=50$} \\
\hline 0.01 & $(1681.6,16152.2)$ & $(424.9,3104.7)$ & $(208.9,673.0)$ & $(134.9,281.1)$ & $(122.7,128.8)$ & $(122.4,121.9)$ & $(122.4,121.9)$ \\
\hline 0.02 & $(759.8,16946.2)$ & $(370.8,1351.5)$ & $(268.4,496.0)$ & $(215.9,419.5)$ & $(160.6,330.5)$ & $(87.6,108.3)$ & $(84.3,83.8)$ \\
\hline 0.05 & $(684.1,4621.2)$ & $(406.9,1037.5)$ & $(324.5,495.4)$ & $(297.5,439.0)$ & $(267.3,406.1)$ & $(177.2,268.6)$ & $(129.7,129.2)$ \\
\hline 0.10 & $(721.5,2022.6)$ & $(508.2,1075.9)$ & $(396.8,569.4)$ & $(379.4,475.3)$ & $(388.9,475.7)$ & $(427.1,500.0)$ & $(555.8,555.3)$ \\
\hline 0.15 & $(448.6,747.2)$ & $(441.0,651.8)$ & $(406.5,535.9)$ & $(375.4,469.7)$ & $(337.5,400.0)$ & $(289.6,290.8)$ & $(288.6,288.1)$ \\
\hline 0.20 & $(366.1,476.2)$ & $(377.3,463.7)$ & $(386.7,446.8)$ & $(393.0,448.2)$ & $(409.9,468.8)$ & $(488.8,549.3)$ & $(643.4,642.9)$ \\
\hline \multicolumn{8}{|c|}{$n=75$} \\
\hline 0.01 & $(1177.0,5012.7)$ & $(634.1,3053.1)$ & $(361.9,830.2)$ & $(369.8,461.3)$ & $(401.1,465.1)$ & $(470.4$ & $(478.1,477.6)$ \\
\hline 0.02 & $(1179.6,49415.1)$ & $(485.7,1803.1)$ & $(344.9,622.8)$ & (309.6 & $(250.5,474.8)$ & $(128.8,208.5)$ & $(109.5,109.0)$ \\
\hline 0.05 & $(917.6,5521.4)$ & $(515.9,1266.9)$ & $(389.5,597.3)$ & $(374.5,504.8)$ & $(371.4,499.4)$ & $(341.8,473.4)$ & $(154.2,153.7)$ \\
\hline 0.10 & $(448.7,747.2)$ & $(456.7,703.9)$ & $(417.4,553.0)$ & $(380.7,478.4)$ & $(340.1,405.0)$ & $(289.8,291.4)$ & $(288.6,288.1)$ \\
\hline 0.15 & $(375.6,485.7)$ & $(390.3,480.9)$ & $(405.4,483.9)$ & $(409.4,484.2)$ & $(397.7,469.0)$ & $(324.5,351.7)$ & $(301.0,300.5)$ \\
\hline 0.20 & $(329.2,409.2)$ & $(368.4,438.7)$ & $(389.5,444.6)$ & $(388.6,426.5)$ & $(375.3,394.4)$ & $(355.6,355.3)$ & $(355.5,355.0)$ \\
\hline \multicolumn{8}{|c|}{$n=100$} \\
\hline 0.01 & $(1017.4,2302.5)$ & $(981.2,2239.4)$ & $(534.4,1569.5)$ & $(328.0,1079.7)$ & $(196.4,548.9)$ & $(155.3,154.8)$ & $(155.3,154.8)$ \\
\hline 0.02 & $(1845.7,58349.4)$ & $(676.2,3053.8)$ & $(437.1,852.0)$ & $(354.9,695.6)$ & $(258.6,504.0)$ & $(171.9,177.7)$ & $(171.0,170.5)$ \\
\hline 0.05 & $(981.7,3229.7)$ & $(655.1,1559.4)$ & $(474.4,721.5)$ & $(428.2,607.5)$ & $(388.2,557.7)$ & $(265.5,347.3)$ & $(224.4,223.9)$ \\
\hline 0.10 & $(386.3,519.9)$ & $(384.1,480.6)$ & $(388.5,451.4)$ & $(396.2,453.4)$ & $(413.7,474.3)$ & $(490.0,551.8)$ & $(643.4,642.9)$ \\
\hline 0.15 & $(330.6,410.1)$ & $(367.7,439.0)$ & $(390.9,446.6)$ & $(390.5,430.4)$ & $(376.9,397.6)$ & $(355.7,355.4)$ & $(355.5,355.0)$ \\
\hline 0.20 & $(317.8,373.3)$ & $(349.0,392.6)$ & $(375.1,407.5)$ & $(376.0,400.9)$ & $(362.2,381.8)$ & $(319.5,323.1)$ & $(312.9,312.4)$ \\
\hline
\end{tabular}

Table 5: $\left(\mathrm{ARL}_{0}, \mathrm{SDRL}_{0}\right)$ for the hypergeometric $n p$ chart with $N=1000, n \in\{25,50,75,100\}, p_{0} \in$ $\{0.01,0.02,0.05,0.10,0.15,0.20\}, m \in\{10,20,50,100,200,1000, \infty\}, K=3$

\begin{tabular}{|c|c|c|c|c|c|c|c|}
\hline$p_{0}$ & $m=10$ & $m=20$ & $m=50$ & $m=100$ & $m=200$ & $m=1000$ & $m \rightarrow \infty$ \\
\hline \multicolumn{8}{|c|}{$n=25$} \\
\hline 0.01 & $(425.0,11105.3)$ & $(216.7,1080.9)$ & $(122.1,323.1)$ & $(85.0,241.2)$ & $(55.6,141.7)$ & $(41.9,41.4)$ & $(41.9,41.4)$ \\
\hline 0.02 & $(430.0,5745.7)$ & $(253.5,824.1)$ & $(135.4,315.9)$ & $(100.7,191.6)$ & $(85.6,97.9)$ & $(84.1,83.6)$ & $(84.1,83.6)$ \\
\hline 0.05 & $(555.7,3054.4)$ & $(301.1,789.3)$ & $(197.7,357.0)$ & $(165.5,216.8)$ & $(158.1,162.2)$ & $(157.6,157.1)$ & $(157.6,157.1)$ \\
\hline 0.10 & $(568.9,3111.0)$ & $(371.5,847.9)$ & $(295.9,439.3)$ & $(277.5,392.0)$ & $(266.5,381.2)$ & $(219.0,329.2)$ & $(116.6,116.1)$ \\
\hline 0.15 & $(745.0,3315.7)$ & $(460.4,994.3)$ & $(363.7,525.2)$ & $(360.9,459.7)$ & $(373.1,465.4)$ & $(411.7,488.5)$ & $(539.0,538.5)$ \\
\hline 0.20 & $(756.3,2071.0)$ & $(531.9,1085.4)$ & $(444.1,642.1)$ & $(414.6,563.3)$ & $(394.5,542.1)$ & $(315.6,447.0)$ & $(200.3,199.8)$ \\
\hline \multicolumn{8}{|c|}{$n=50$} \\
\hline 0.01 & $(553.0,12194.3)$ & $(226.8,1025.1)$ & $(152.3,377.1)$ & $(104.7,196.2)$ & $(92.0,103.7)$ & & $(90.8$, \\
\hline 0.02 & $(576.6$ & (293. & $(201.2,3$ & $(187.3$ & $(167.7$ & $(96.9,1$ & \\
\hline 0.05 & $(586.0,3$ & (373.7, & $(277.0,4$ & $(267.1$, & $(265.8$ & $(275.4$, & (424.1, \\
\hline 0.10 & $(707.7,2$ & $(451.4,9$ & $(357.7,511.8)$ & $(342.4,4$ & $(356.5,387.5)$ & $(401.0,404.4)$ & $(406.8,406.3)$ \\
\hline 0.15 & $(449.6,7$ & $(422.7,622.6)$ & $(376.0,479.2)$ & $(359.0,436.6)$ & $(341.2,415.9)$ & $(278.9,333.9)$ & $(224.0,223.5)$ \\
\hline 0.20 & $(364.5,461.3)$ & $(375.7,445.2)$ & $(390.2,437.3)$ & $(408.0,443.6)$ & $(435.5$ & $(477$. & $(479.7,479.2)$ \\
\hline \multicolumn{8}{|c|}{$n=75$} \\
\hline 0.01 & $(779.5,13477.1)$ & $(273.4,1147.1)$ & $(196.8,366.9)$ & $(200.5,230.1)$ & $(217.3,227.9)$ & $(230.4,229.9)$ & $(230.4,229.9)$ \\
\hline 0.02 & $(629.2,4643.4)$ & $(346.0,905.0)$ & $(255.1,386.5)$ & $(248.4,344.3)$ & $(247.1,342.8)$ & $(245.5,341.6)$ & $(412.5,412.0)$ \\
\hline 0.05 & $(668.4,3370.7)$ & $(413.5,898.5)$ & $(318.5,464.1)$ & $(303.7,359.7)$ & $(321.3,351.2)$ & $(363.0,366.2)$ & $(368.2,367.7)$ \\
\hline 0.10 & $(475.2,787.1)$ & $(420.1,627.6)$ & $(365.4,459.7)$ & $(351.0,410.3)$ & $(348.5,403.0)$ & $(347.3,401.5)$ & $(562.4,561.9)$ \\
\hline 0.15 & $(370.5,510.1)$ & $(383.3,498.1)$ & $(387.2,464.9)$ & $(379.1,425.1)$ & $(386.0,416.2)$ & $(431.2,440.7)$ & $(450.4,449.9)$ \\
\hline 0.20 & $(338.8,422.7)$ & $(359.9,421.4)$ & $(364.9,398.1)$ & $(361.5,381.4)$ & $(359.9,376.4)$ & $(358.8,374.5)$ & $(608.6,608.1)$ \\
\hline \multicolumn{8}{|c|}{$n=100$} \\
\hline 0.01 & $(692.7,22439.4)$ & $(344.8,1218.5)$ & & $(203.2,389.9)$ & $(161.2,324.5)$ & $(86.6,11$ & $(81.1,80.6)$ \\
\hline 0.02 & $(683.0,5711.7)$ & $(372.6,1030.5)$ & $(281.9,438.7)$ & $(277.8,386.1)$ & $(271.7,380.3)$ & $(261.1,371.7)$ & $(95.8,95.3)$ \\
\hline 0.05 & $(719.7,2856.2)$ & $(451.0,972.2)$ & $(357.7,513.2)$ & $(338.9,404.3)$ & $(354.0,386.5)$ & $(400.3,404.1)$ & $(406.8,406.3)$ \\
\hline 0.10 & $(372.6,508.1)$ & $(392.7,516.0)$ & $(390.5,486.0)$ & $(373.2,436.8)$ & $(352.8,381.8)$ & $(335.1,334.7)$ & $(335.1,334.6)$ \\
\hline 0.15 & $(339.1,419.5)$ & $(356.2,418.6)$ & $(370.0,412.4)$ & $(382.5,417.2)$ & $(403.1,434.4)$ & $(469.8,483.8)$ & $(501.0,500.5)$ \\
\hline 0.20 & $(312.6,363.2)$ & $(339.1,374.4)$ & $(363.7,384.4)$ & $(378.7,393.3)$ & $(394.1,403.1)$ & $(415.7,415.5)$ & $(416.4,415.9)$ \\
\hline
\end{tabular}


Table 6: $\left(\mathrm{ARL}_{0}, \mathrm{SDRL}_{0}\right)$ for the hypergeometric $n p$ chart with $N=2000, n \in\{25,50,75,100\}, p_{0} \in$ $\{0.01,0.02,0.05,0.10,0.15,0.20\}, m \in\{10,20,50,100,200,1000, \infty\}, K=3$

\begin{tabular}{|c|c|c|c|c|c|c|c|}
\hline$p_{0}$ & $m=10$ & $m=20$ & $m=50$ & $m=100$ & $m=200$ & $m=1000$ & $m \rightarrow \infty$ \\
\hline \multicolumn{8}{|c|}{$n=25$} \\
\hline 0.01 & $(340.3,5635.8)$ & $(188.2,825.8)$ & $(109.9,280.1)$ & $(77.9,210.1)$ & $(52.4,125.0)$ & $(40.3,39.8)$ & $(40.3,39.8)$ \\
\hline 0.02 & $(369.1,4118.4)$ & $(227.0,695.2)$ & $(124.9,280.6)$ & $(94.5,172.5)$ & $(81.4,95.4)$ & $(79.6,79.1)$ & $(79.6,79.1)$ \\
\hline 0.05 & $(498.9,2916.7)$ & $(276.2,701.6)$ & $(186.6,325.5)$ & $(158.5,212.0)$ & $(149.1,154.6)$ & $(148.1,147.6)$ & $(148.1,147.6)$ \\
\hline 0.10 & $(591.1,2779.4)$ & $(367.5,845.1)$ & $(290.9,425.4)$ & $(277.9,379.2)$ & $(268.7,370.9)$ & $(246.2,350.4)$ & $(110.8,110.3)$ \\
\hline 0.15 & $(688.1,2951.6)$ & $(459.2,1017.7)$ & $(354.3,508.3)$ & $(353.4,440.0)$ & $(370.4,446.3)$ & $(425.8,474.5)$ & $(501.1,500.6)$ \\
\hline 0.20 & $(697.3,1871.5)$ & $(546.4,1106.0)$ & $(431.4,618.5)$ & $(417.6,550.8)$ & $(410.7,543.3)$ & $(366.4,501.0)$ & $(189.7,189.2)$ \\
\hline \multicolumn{8}{|c|}{$n=50$} \\
\hline 0.01 & $(381.7,4543.6)$ & $(232.4,721.7)$ & $(127.0,287.9)$ & $(95.8,176.4)$ & $(82.3,96.8)$ & $(80.5,80.0)$ & $(80.5,80.0)$ \\
\hline 0.02 & $(441.9,3144.9)$ & $(243.4,668.8)$ & $(187.8,299.6)$ & $(182.5,279.9)$ & $(166.6,265.0)$ & $(118.8,206.7)$ & $(61.4,60.9)$ \\
\hline 0.05 & $(543.5,2800.1)$ & $(321.5,747.2)$ & $(254.1,373.5)$ & $(252.0,316.6)$ & $(263.9,321.0)$ & $(300.3,340.0)$ & $(362.4,361.9)$ \\
\hline 0.10 & $(636.7,1990.0)$ & $(426.5,880.2)$ & $(339.7,475.8)$ & $(320.7,375.0)$ & $(329.0,346.6)$ & $(353.1,353.0)$ & $(353.8,353.3)$ \\
\hline 0.15 & $(428.8,707.0)$ & $(407.7,605.1)$ & $(366.9,459.0)$ & $(352.6,414.7)$ & $(350.4,409.5)$ & $(337.3,398,3)$ & $(199.6,199.1)$ \\
\hline 0.20 & $(364.9,482.9)$ & $(368.1,447.1)$ & $(390.6,455.4)$ & $(395.5,434.2)$ & $(405.4,420.5)$ & $(419.5,419.0)$ & $(419.6,419.1)$ \\
\hline \multicolumn{8}{|c|}{$n=75$} \\
\hline 0.01 & $(452.3,4319.3)$ & $(250.0,728.1)$ & $(164.7,286.6)$ & $(163.6,182.8)$ & $(172.4,177.1)$ & $(178.6,178.1)$ & $(178.6,178.1)$ \\
\hline 0.02 & $(486.7,2971.0)$ & $(290.3,727.6)$ & $(214.4,321.1)$ & $(219.1,280.3)$ & $(227.9,283.7)$ & $(265.8,301.1)$ & $(316.4,315.9)$ \\
\hline 0.05 & $(602.2,2615.0)$ & $(367.5,788.7)$ & $(288.2,414.0)$ & $(270.7,319.0)$ & $(278.5,291.6)$ & $(295.3,294.9)$ & $(295.5,295.0)$ \\
\hline 0.10 & $(455.6,747.3)$ & $(399.7,581.3)$ & $(356.8,440.9)$ & $(352.0,403.3)$ & $(367.2,407.0)$ & $(422.1,438.1)$ & $(450.4,449.9)$ \\
\hline 0.15 & $(374.9,516.2)$ & $(381.7,491.2)$ & $(366.9,436.7)$ & $(354.9,392.6)$ & $(353.5,367.8)$ & $(366.9,366.8)$ & $(367.0,366.5)$ \\
\hline 0.20 & $(331.8,408.7)$ & $(361.4,421.7)$ & $(371.2,411.9)$ & $(384.8,418.2)$ & $(409.0,437.6)$ & $(472.8,481.6)$ & $(492.0,491.5)$ \\
\hline \multicolumn{8}{|c|}{$n=100$} \\
\hline 0.01 & $(523.6,4384.9)$ & $(274.0,755.6)$ & $(209.0,339.4)$ & $(189.3,303.2)$ & $(166.7,279.7)$ & $(104.1,187.2)$ & $(65.1,64.6)$ \\
\hline 0.02 & $(523.3,3210.2)$ & $(299.2,725.9)$ & $(240.6,353.9)$ & $(236.7,296.4)$ & $(251.6,301.2)$ & $(269.7,320.6)$ & $(331.0,330.5)$ \\
\hline 0.05 & $(663.9,2493.2)$ & $(420.4,876.2)$ & $(323.3,460.7)$ & $(297.1,355.4)$ & $(293.6,307.5)$ & $(302.2,301.7)$ & $(302.2,301.7)$ \\
\hline 0.10 & $(400.0,597.2)$ & $(390.3,544.9)$ & $(363.2,452.5)$ & $(346.1,405.6)$ & $(325.9,374.3)$ & $(270.0,283.4)$ & $(257.4,256.9)$ \\
\hline 0.15 & $(336.5,421.8)$ & $(356.1,427.1)$ & $(369.8,420.2)$ & $(374.4,410.7)$ & $(375.4,393.7)$ & $(378.3,377.9)$ & $(378.5,378.0)$ \\
\hline 0.20 & $(315.1,376.4)$ & $(348.0,396.4)$ & $(367.3,403.5)$ & $(371.8,400.2)$ & $(360.8,381.5)$ & $(323.8,326.7)$ & $(318.8,318.3)$ \\
\hline
\end{tabular}

Table 7: $\left(\mathrm{ARL}_{0}, \mathrm{SDRL}_{0}\right)$ for the hypergeometric $n p$ chart with $N=5000, n \in\{25,50,75,100\}, p_{0} \in$ $\{0.01,0.02,0.05,0.10,0.15,0.20\}, m \in\{10,20,50,100,200,1000, \infty\}, K=3$

\begin{tabular}{|c|c|c|c|c|c|c|c|}
\hline$p_{0}$ & $m=10$ & $m=20$ & $m=50$ & $m=100$ & $m=200$ & $m=1000$ & $m \rightarrow \infty$ \\
\hline \multicolumn{8}{|c|}{$n=25$} \\
\hline 0.01 & $(303.9,4190.7)$ & $(174.6,719.1)$ & $(135.4,310.6)$ & $(88.6,228.3)$ & $(54.8,133.7)$ & $(39.4,38.9)$ & $(39.4,38.9)$ \\
\hline 0.02 & $(339.6,3460.6)$ & $(213.4,633.4)$ & $(138.7,305.8)$ & $(96.4,183.8)$ & $(79.4,96.1)$ & $(77.1,76.6)$ & $(77.1,76.6)$ \\
\hline 0.05 & $(468.5,2683.6)$ & $(262.8,656.1)$ & $(191.0,338.5)$ & $(156.1,214.5)$ & $(144.4,152.1)$ & $(142.9,142.4)$ & $(142.9,142.4)$ \\
\hline 0.10 & $(606.4,2830.7)$ & $(353.1,833.9)$ & $(279.0,406.1)$ & $(275.8,369.9)$ & $(270.9,365.2)$ & $(262.2,358.1)$ & $(107.6,107.1)$ \\
\hline 0.15 & $(680.1,2759.3)$ & $(457.2,968.2)$ & $(354.7,507.6)$ & $(347.0,427.2)$ & $(365.6,433.4)$ & $(427.6,462.5)$ & $(480.2,479.7)$ \\
\hline 0.20 & $(665.2,1764.2)$ & $(523.6,1051.6)$ & $(430.8,614.3)$ & $(420.5,544.1)$ & $(415.0,537.4)$ & $(396.7,522.0)$ & $(183.8,183.3)$ \\
\hline \multicolumn{8}{|c|}{$n=50$} \\
\hline 0.01 & $(319.8,3080.3)$ & $(204.3,592.3)$ & $(134.0,291.5)$ & $(93.9,176.0)$ & $(77.6,93.3)$ & $(75.4,74.9)$ & $(75.4,74.9)$ \\
\hline 0.02 & $(384.5,2634.4)$ & $(219.2,574.9)$ & $(187.3,287.8)$ & $(177.2,261.3)$ & $(167.1,252.6)$ & $(133.8,218.6)$ & $(58.2,57.7)$ \\
\hline 0.05 & $(538.1,2572.9)$ & $(329.1,711.6)$ & $(245.7,357.6)$ & $(244.2,298.7)$ & $(254.8,300.6)$ & $(299.0,320.9)$ & $(331.8,331.3)$ \\
\hline 0.10 & $(659.8,2252.7)$ & $(427.8,874.1)$ & $(331.1,468.2)$ & $(308.7,362.1)$ & $(311.8,325.4)$ & $(326.7,326.3)$ & $(326.8,326.3)$ \\
\hline 0.15 & $(451.0,725.3)$ & $(407.8,601.2)$ & $(365.2,455.3)$ & $(356.0,416.8)$ & $(364.3,421.3)$ & $(399.1,456.2)$ & $(541.7,541.2)$ \\
\hline 0.20 & $(370.3,494.8)$ & $(383.6,492.7)$ & $(393.0,476.6)$ & $(392.9,446.2)$ & $(387.7,405.8)$ & $(388.6,388.1)$ & $(388.6,388.1)$ \\
\hline \multicolumn{8}{|c|}{$n=75$} \\
\hline 0.01 & $(353.9,2705.0)$ & $(213.5,641.0)$ & $(155.9,268.2)$ & $(146.9,166.0)$ & $(152.8,155.5)$ & $(156.6,156.1)$ & $(156.6,156.1)$ \\
\hline 0.02 & $(412.0,2469.1)$ & $(267.0,636.5)$ & $(199.8,292.9)$ & $(205.0,251.3)$ & $(216.8,254.8)$ & $(255.1,269.3)$ & $(274.8,274.3)$ \\
\hline 0.05 & $(535.9,2325.4)$ & $(356.1,743.5)$ & $(275.5,392.8)$ & $(255.0,303.2)$ & $(255.0,264.6)$ & $(261.9,261.5)$ & $(262.0,261.5)$ \\
\hline 0.10 & $(418.8,685.9)$ & $(380.8,543.8)$ & $(346.6,421.7)$ & $(341.9,384.6)$ & $(353.6,378.5)$ & $(392.6,395.6)$ & $(398.4,397.9)$ \\
\hline 0.15 & $(361.7,499.9)$ & $(370.6,483.0)$ & $(363.0,427.4)$ & $(351.6,387.5)$ & $(345.1,359.4)$ & $(340.4,341.4)$ & $(327.3,326.8)$ \\
\hline 0.20 & $(334.5,407.8)$ & $(357.0,409.2)$ & $(368.4,401.3)$ & $(382.8,406.4)$ & $(400.7,416.4)$ & $(434.4,435.1)$ & $(436.7,436.2)$ \\
\hline \multicolumn{8}{|c|}{$n=100$} \\
\hline 0.01 & $(384.5,2634.4)$ & $(243.2,668.7)$ & $(187.3,287.8)$ & $(177.2,261.4)$ & $(167.1,252.6)$ & $(133.8,218.6)$ & $(58.2,57.7)$ \\
\hline 0.02 & $(452.3,2272.3)$ & $(284.5,649.7)$ & $(216.0,320.2)$ & $(215.6,259.2)$ & $(231.0,259.9)$ & $(267.6,272.8)$ & $(275.4,274.9)$ \\
\hline 0.05 & $(585.3,2291.5)$ & $(383.8,774.6)$ & $(304.2,427.0)$ & $(278.9,342.6)$ & $(262.3,280.8)$ & $(258.1,257.6)$ & $(258.1,257.6)$ \\
\hline 0.10 & $(391.2,571.8)$ & $(378.6,526.1)$ & $(348.9,428.0)$ & $(335.5,385.1)$ & $(326.2,370.3)$ & $(288.1,328.3)$ & $(223.1,222.6)$ \\
\hline 0.15 & $(334.1,420.4)$ & $(352.7,422.7)$ & $(367.2,422.7)$ & $(365.4,407.8)$ & $(352.1,377.4)$ & $(325.7,326.0)$ & $(324.7,324.2)$ \\
\hline 0.20 & $(323.4,386.7)$ & $(347.3,396.6)$ & $(363.8,396.8)$ & $(363.1,385.1)$ & $(355.8,373.9)$ & $(320.9,337.6)$ & $(275.3,274.8)$ \\
\hline
\end{tabular}


Table 8: $\left(\mathrm{ARL}_{0}, \mathrm{SDRL}_{0}\right)$ for the hypergeometric $n p$ chart with $N=10000, n \in\{25,50,75,100\}, p_{0} \in$ $\{0.01,0.02,0.05,0.10,0.15,0.20\}, m \in\{10,20,50,100,200,1000, \infty\}, K=3$

\begin{tabular}{|c|c|c|c|c|c|c|c|}
\hline$p_{0}$ & $m=10$ & $m=20$ & $m=50$ & $m=100$ & $m=200$ & $m=1000$ & $m \rightarrow \infty$ \\
\hline \multicolumn{8}{|c|}{$n=25$} \\
\hline 0.01 & $(293.4,3841.1)$ & $(170.5,688.8)$ & $(132.7,302.8)$ & $(87.1,222.8)$ & $(54.2,130.8)$ & $(39.1,38.7)$ & $(39.1,38.6)$ \\
\hline 0.02 & $(330.8,3277.2)$ & $(209.2,614.9)$ & $(136.5,299.1)$ & $(95.3,180.2)$ & $(78.6,94.8)$ & $(76.3,75.8)$ & $(76.3,75.8)$ \\
\hline 0.05 & $(459.0,2594.6)$ & $(258.6,642.0)$ & $(188.4,332.5)$ & $(154.3,211.3)$ & $(142.7,150.2)$ & $(141.2,140.7)$ & $(141.2,140.7)$ \\
\hline 0.10 & $(595.7,2760.7)$ & $(347.9,818.3)$ & $(277.0,410.7)$ & $(272.1,364.7)$ & $(273.6,364.8)$ & $(267.2,359.8)$ & $(106.5,106.0)$ \\
\hline 0.15 & $(669.7,2770.1)$ & $(450.6,951.6)$ & $(350.0,500.2)$ & $(349.7,425.6)$ & $(365.6,430.0)$ & $(427.2,458.2)$ & $(473.5,473.0)$ \\
\hline 0.20 & $(692.9,1995.0)$ & $(520.8,1096.7)$ & $(425.1,605.5)$ & $(414.9,536.4)$ & $(416.2,535.2)$ & $(406.4,527.3)$ & $(181.9,181.4)$ \\
\hline \multicolumn{8}{|c|}{$n=50$} \\
\hline 0.01 & $(303.2,2752.6)$ & $(196.3,565.5)$ & $(129.8,278.7)$ & $(91.6,169.1)$ & $(76.7,95.6)$ & $(73.9,73.4)$ & $(73.9,73.4)$ \\
\hline 0.02 & $(368.0,2432.0)$ & $(234.9,636.7)$ & $(181.7,277.9)$ & $(172.0,252.7)$ & $(162.3,244.3)$ & $(138.9,221.4)$ & $(57.3,56.8)$ \\
\hline 0.05 & $(518.8,2436.4)$ & $(319.3,684.9)$ & $(239.1,346.8)$ & $(237.8,291.3)$ & $(251.7,294.2)$ & $(296.5,314.0)$ & $(322.5,322.0)$ \\
\hline 0.10 & $(638.5,2157.2)$ & $(415.8,844.6)$ & $(326.8,455.6)$ & $(305.0,358.4)$ & $(306.2,318.9)$ & $(318.4,318.0)$ & $(318.5,318.0)$ \\
\hline 0.15 & $(438.9,704.5)$ & $(397.4,584.7)$ & $(359.8,451.3)$ & $(355.7,416.1)$ & $(367.3,423.7)$ & $(415.4,466.4)$ & $(527.0,526.5)$ \\
\hline 0.20 & $(361.1,481.8)$ & $(388.4,498.3)$ & $(397.2,486.4)$ & $(392.6,449.3)$ & $(381.7,401.0)$ & $(379.1,378.6)$ & $(379.1,378.6)$ \\
\hline \multicolumn{8}{|c|}{$n=75$} \\
\hline 0.01 & $(329.1,2355.4)$ & $(202.2,589.4)$ & $(149.2,252.5)$ & $(141.0,158.9)$ & $(147.3,149.4)$ & $(150.3,149.8)$ & $(150.3,149.8)$ \\
\hline 0.02 & $(388.2,2232.2)$ & $(254.4,596.0)$ & $(194.3,293.4)$ & $(196.5,241.0)$ & $(211.5,245.4)$ & $(248.8,258.9)$ & $(262.9,262.4)$ \\
\hline 0.05 & $(514.8,2333.4)$ & $(341.5,719.4)$ & $(271.6,391.4)$ & $(251.2,302.9)$ & $(247.3,257.2)$ & $(252.1,251.6)$ & $(252.1,251.6)$ \\
\hline 0.10 & $(402.8,657.8)$ & $(368.5,522.9)$ & $(342.9,414.9)$ & $(337.4,377.4)$ & $(347.1,368.1)$ & $(379.9,381.3)$ & $(383.0,382.5)$ \\
\hline 0.15 & $(376.8,528.5)$ & $(377.1,489.8)$ & $(358.4,420.2)$ & $(348.2,382.8)$ & $(342.1,356.6)$ & $(337.7,338.9)$ & $(315.5,315.0)$ \\
\hline 0.20 & $(322.7,392.7)$ & $(349.4,399.2)$ & $(367.0,396.2)$ & $(378.7,399.0)$ & $(393.9,406.2)$ & $(419.2,419.3)$ & $(420.3,419.8)$ \\
\hline \multicolumn{8}{|c|}{$n=100$} \\
\hline 0.01 & $(351.3,2230.6)$ & $(226.4,604.4)$ & $(176.0,268.0)$ & $(166.7,244.0)$ & $(164.4,242.0)$ & $(143.7,223.0)$ & $(56.3,55.8)$ \\
\hline 0.02 & $(420.8,2292.2)$ & $(271.5,645.6)$ & $(215.6,318.5)$ & $(209.0,248.5)$ & $(223.9,247.6)$ & $(255.9,258.5)$ & $(260.1,259.6)$ \\
\hline 0.05 & $(582.1,2202.4)$ & $(379.4,777.5)$ & $(298.6,416.9)$ & $(273.3,337.6)$ & $(253.7,275.2)$ & $(245.6,245.1)$ & $(245.6,245.1)$ \\
\hline 0.10 & $(386.8,585.3)$ & $(372.7,512.1)$ & $(346.1,422.6)$ & $(331.0,376.8)$ & $(325.6,365.3)$ & $(306.6,347.5)$ & $(213.2,212.7)$ \\
\hline 0.15 & $(339.2,422.9)$ & $(354.2,421.7)$ & $(363.5,416.3)$ & $(360.5,401.7)$ & $(347.3,375.1)$ & $(311.9,313.6)$ & $(309.3,308.8)$ \\
\hline 0.20 & $(322.9,392.5)$ & $(348.5,398.7)$ & $(360.2,390.9)$ & $(360.2,379.1)$ & $(355.6,370.3)$ & $(336.0,352.4)$ & $(262.7,262.2)$ \\
\hline
\end{tabular}

Table 9: $\left(\mathrm{ARL}_{0}, \quad \mathrm{SDRL}_{0}\right)$ for the binomial $n p$ chart with $n \in\{25,50,75,100\}, \quad p_{0} \in$ $\{0.01,0.02,0.05,0.10,0.15,0.20\}, m \in\{10,20,50,100,200,1000, \infty\}, K=3$

\begin{tabular}{|c|c|c|c|c|c|c|c|}
\hline$p_{0}$ & $m=10$ & $m=20$ & $m=50$ & $m=100$ & $m=200$ & $m=1000$ & $m \rightarrow \infty$ \\
\hline \multicolumn{8}{|c|}{$n=25$} \\
\hline 0.01 & $(283.6,3538.1)$ & $(166.6,660.7)$ & $(130.1,295.3)$ & $(85.7,217.5)$ & $(53.6,128.0)$ & $(38.8,38.4)$ & $(38.8,38.3)$ \\
\hline 0.02 & $(322.3,3153.5)$ & $(205.2,597.3)$ & $(134.4,292.6)$ & $(94.1,176.7)$ & $(77.8,93.5)$ & $(75.5,75.0)$ & $(75.5,75.0)$ \\
\hline 0.05 & $(471.5,2957.7)$ & $(254.6,636.2)$ & $(185.8,326.7)$ & $(152.4,208.2)$ & $(141.0,148.4)$ & $(139.6,139.1)$ & $(139.6,139.1)$ \\
\hline 0.10 & $(614.0,2845.8)$ & $(362.8,808.0)$ & $(285.9,412.8)$ & $(277.3,366.1)$ & $(276.2,364.3)$ & $(274.9,363.3)$ & $(442.2,441.7)$ \\
\hline 0.15 & $(712.7,2928.1)$ & $(448.2,983.7)$ & $(345.4,493.0)$ & $(345.2,420.7)$ & $(365.4,426.5)$ & $(426.3,453.6)$ & $(467.0,466.5)$ \\
\hline 0.20 & $(711.7,1958.3)$ & $(534.5,1084.6)$ & $(432.7,605.7)$ & $(418.9,536.6)$ & $(417.3,532.8)$ & $(415.9,531.6)$ & $(649.3,648.8)$ \\
\hline \multicolumn{8}{|c|}{$n=50$} \\
\hline 0.01 & $(288.0,2477.0)$ & $(188.8,533.4)$ & $(125.9,266.8)$ & $(89.5,162.7)$ & $(75.2,93.0)$ & $(72.4,71.9)$ & $(72.4,71.9)$ \\
\hline 0.02 & $(352.7,2252.6)$ & $(227.0,607.1)$ & $(176.4,268.6)$ & $(167.0,244.6)$ & $(164.7,242.5)$ & $(143.9,223.5)$ & $(56.3,55.8)$ \\
\hline 0.05 & $(500.6,2310.1)$ & $(309.9,664.5)$ & $(244.4,354.1)$ & $(237.3,287.5)$ & $(252.1,289.7)$ & $(293.3,306.9)$ & $(313.6,313.1)$ \\
\hline 0.10 & $(622.1,2171.5)$ & $(404.8,817.7)$ & $(325.3,458.6)$ & $(301.3,355.0)$ & $(300.6,312.8)$ & $(310.5,310.0)$ & $(310.6,310.1)$ \\
\hline 0.15 & $(449.8,747.5)$ & $(403.4,587.3)$ & $(361.2,449.0)$ & $(360.1,418.7)$ & $(372.2,426.1)$ & $(426.4,469.6)$ & $(512.9,512.4)$ \\
\hline 0.20 & $(389.0,532.5)$ & $(399.9,521.5)$ & $(400.4,493.1)$ & $(388.3,446.4)$ & $(375.8,396.6)$ & $(369.8,369.3)$ & $(369.8,369.3)$ \\
\hline \multicolumn{8}{|c|}{$n=75$} \\
\hline 0.01 & $(307.1,2072.2)$ & $(192.0,544.4)$ & $(143.0,238.5)$ & $(138.2,156.7)$ & $(142.2,143.8)$ & $(144.5,144.0)$ & $(144.5,144.0)$ \\
\hline 0.02 & $(366.5,2077.0)$ & $(242.8,562.8)$ & $(195.0,283.1)$ & $(194.3,234.4)$ & $(206.3,236.4)$ & $(241.9,248.9)$ & $(251.8,251.3)$ \\
\hline 0.05 & $(520.7,2211.7)$ & $(345.9,701.1)$ & $(271.6,389.3)$ & $(245.6,297.3)$ & $(239.8,249.8)$ & $(242.8,242.3)$ & $(242.8,242.3)$ \\
\hline 0.10 & $(440.5,715.2)$ & $(380.5,543.1)$ & $(342.3,421.7)$ & $(335.1,380.8)$ & $(340.7,360.3)$ & $(366.9,367.3)$ & $(368.5,368.0)$ \\
\hline 0.15 & $(362.8,507.1)$ & $(366.5,475.6)$ & $(357.0,414.4)$ & $(348.3,381.6)$ & $(340.4,355.4)$ & $(335.4,336.4)$ & $(351.2,350.7)$ \\
\hline 0.20 & $(341.1,423.9)$ & $(354.0,409.7)$ & $(365.7,400.4)$ & $(377.5,401.3)$ & $(388.5,401.5)$ & $(404.3,404.0)$ & $(404.7,404.2)$ \\
\hline \multicolumn{8}{|c|}{$n=100$} \\
\hline 0.01 & $(322.8,1942.9)$ & $(211.6,549.4)$ & $(166.9,258.3)$ & $(166.6,236.0)$ & $(161.8,232.0)$ & $(153.0,224.8)$ & $(54.4,53.9)$ \\
\hline 0.02 & $(405.8,2275.0)$ & $(255.5,600.8)$ & $(204.2,299.1)$ & $(202.7,238.6)$ & $(216.7,236.2)$ & $(243.8,245.0)$ & $(246.2,245.7)$ \\
\hline 0.05 & $(569.1,2046.3)$ & $(373.5,739.4)$ & $(293.2,407.4)$ & $(267.4,332.9)$ & $(245.9,271.1)$ & $(234.0,233.5)$ & $(234.0,233.5)$ \\
\hline 0.10 & $(395.6,591.8)$ & $(379.0,526.7)$ & $(345.1,419.2)$ & $(330.8,373.4)$ & $(327.4,362.1)$ & $(326.5,360.7)$ & $(498.7,498.2)$ \\
\hline 0.15 & $(326.7,404.4)$ & $(347.4,412.4)$ & $(359.6,408.7)$ & $(356.6,395.9)$ & $(343.1,372.4)$ & $(301.3,306.1)$ & $(294.9,294.4)$ \\
\hline 0.20 & $(322.8,386.5)$ & $(347.6,397.4)$ & $(359.3,389.2)$ & $(357.9,374.4)$ & $(356.0,367.1)$ & $(355.2,365.3)$ & $(547.2,546.7)$ \\
\hline
\end{tabular}


Table 10: Selected results of the simulation study regarding $\mathrm{ARL}_{0}$

\begin{tabular}{lcclcclcc}
\hline$m, N, n, p_{0}$ & $\mathrm{ARL}_{0}$ & $\mathrm{ARL}_{0}^{\text {sim }}$ & $m, N, n, p_{0}$ & $\mathrm{ARL}_{0}$ & $\mathrm{ARL}_{0}^{\text {sim }}$ & $m, N, n, p_{0}$ & $\mathrm{ARL}_{0}$ & $\mathrm{ARL}_{0}^{\text {sim }}$ \\
\hline $10,100,25,0.01$ & $\infty$ & $\infty$ & $20,100,50,0.02$ & $\infty$ & $\infty$ & $50,100,75,0.05$ & 1220.1 & 1210.9 \\
$100,200,50,0.1$ & 476.0 & 471.0 & $200,200,75,0.15$ & 440.4 & 438.3 & $1000,200,100,0.2$ & 422.8 & 422.5 \\
$10,500,25,0.01$ & 851.8 & 815.5 & $20,500,50,0.02$ & 370.8 & 369.3 & $50,500,75,0.05$ & 389.5 & 400.2 \\
$100,1000,50,0.1$ & 342.4 & 340.5 & $200,1000,75,0.15$ & 386.0 & 389.5 & $1000,1000,100,0.2$ & 415.7 & 415.2 \\
$10,2000,25,0.01$ & 340.3 & 346.2 & $20,2000,50,0.02$ & 243.4 & 243.9 & $50,2000,75,0.05$ & 288.2 & 282.0 \\
$100,5000,50,0.1$ & 308.7 & 308.9 & $200,5000,75,0.15$ & 345.1 & 346.2 & $1000,5000,100,0.2$ & 320.9 & 319.2 \\
$10,10000,25,0.01$ & 293.4 & 295.7 & $20,10000,50,0.02$ & 234.9 & 234.2 & $50,10000,75,0.05$ & 271.6 & 269.2 \\
\hline
\end{tabular}

Table 11: Values of $m$ for $n \in\{25,50,75,100\}, p_{0} \in\{0.01,0.02,0.05,0.10,0.15,0.20\}, N \in$ $\{100,200,500,1000,2000,5000,10000\}, K=3$, satisfying $\Delta=\frac{\left|\mathrm{ARL}_{0, m}-\mathrm{ARL}_{0, \infty}\right|}{\mathrm{ARL}_{0, \infty}} \leq 0.05$

\begin{tabular}{|c|c|c|c|c|c|c|}
\hline$N \backslash p_{0}$ & 0.01 & 0.02 & 0.05 & 0.10 & 0.15 & 0.20 \\
\hline \multicolumn{7}{|c|}{$n=25$} \\
\hline 100 & - & - & 1101 & 78 & 70 & 277 \\
\hline 200 & 18 & 106 & 112 & 407 & 640 & 242 \\
\hline 500 & 310 & 134 & 83 & 2751 & $>10000$ & 1640 \\
\hline 1000 & 343 & 150 & 100 & $>10000$ & 14 & 6763 \\
\hline 2000 & 371 & 159 & 117 & $>10000$ & 15 & $>10000$ \\
\hline 5000 & 382 & 171 & 125 & $>10000$ & 15 & $>10000$ \\
\hline 10000 & 383 & 171 & 125 & $>10000$ & 16 & $>10000$ \\
\hline \multicolumn{7}{|c|}{$n=50$} \\
\hline 100 & - & - & - & 41 & 951 & $>10000$ \\
\hline 200 & - & 29 & 144 & 160 & 3331 & 151 \\
\hline 500 & 119 & 931 & 2926 & 16 & 433 & 7358 \\
\hline 1000 & 151 & 2822 & 15 & 24 & 3251 & 340 \\
\hline 2000 & 164 & 6904 & 16 & 33 & $>10000$ & 111 \\
\hline 5000 & 175 & $>10000$ & 18 & 40 & $>10000$ & 10 \\
\hline 10000 & 180 & $>10000$ & 18 & 43 & 8060 & 10 \\
\hline \multicolumn{7}{|c|}{$n=75$} \\
\hline 100 & - & - & 104 & 15 & 10 & 189 \\
\hline 200 & - & - & 46 & 254 & 411 & 409 \\
\hline 500 & 576 & 1572 & $>10000$ & 453 & 1281 & 218 \\
\hline 1000 & 210 & 15 & 521 & $>10000$ & 892 & $>10000$ \\
\hline 2000 & 147 & 15 & 217 & 10 & 10 & 837 \\
\hline 5000 & 38 & 18 & 49 & 11 & 303 & 332 \\
\hline 10000 & 38 & 18 & 64 & 13 & $3,4,>10000$ & 250 \\
\hline \multicolumn{7}{|c|}{$n=100$} \\
\hline 100 & - & - & - & - & - & - \\
\hline 200 & - & - & 30 & 105 & 17 & 49 \\
\hline 500 & 313 & 597 & 1923 & 7884 & 11 & 10 \\
\hline 1000 & 1112 & $>10000$ & 26 & 211 & 1193 & 216 \\
\hline 2000 & 3834 & 16 & 51 & 984 & 18 & 10 \\
\hline 5000 & $>10000$ & 19 & 122 & 6340 & 10 & 3531 \\
\hline 10000 & $>10000$ & 20 & 160 & $>10000$ & 455 & $4,>10000$ \\
\hline
\end{tabular}


Table 12: $\left(K^{\prime}, \mathrm{ARL}_{0}, \mathrm{SDRL}_{0}\right)$ for the hypergeometric $n p$ chart with $N=100, n \in\{25,50,75,100\}, p_{0} \in$ $\{0.01,0.02,0.05,0.10,0.15,0.20\}, m \in\{10,20,50,100,200\}$

\begin{tabular}{|c|c|c|c|c|c|}
\hline$p_{0}$ & $m=10$ & $m=20$ & $m=50$ & $m=100$ & $m=200$ \\
\hline \multicolumn{6}{|c|}{$n=25$} \\
\hline 0.01 & - & - & - & - & - \\
\hline 0.02 & - & - & - & - & - \\
\hline 0.05 & $(3.83,1301.2,1406.1)$ & $(3.84,1371.3,1413.4)$ & $(3.90,1414.9,1416.4)$ & $(3.93,1417.0,1416.5)$ & $(3.95,1417.0,1416.5)$ \\
\hline 0.10 & $(2.59,465.8,3177.5)$ & $(2.78,506.0,1689.4)$ & $(2.92,491.8,989.2)$ & $(2.99,488.9,650.0)$ & $(3.02,485.9,495.0)$ \\
\hline 0.15 & $(2.77,495.7,1359.1)$ & $(2.83,523.5,1287.2)$ & $(2.95,531.4,897.0)$ & $(3.00,536.2,666.4)$ & $(3.03,535.4,547.5)$ \\
\hline 0.20 & $(3.08,778.1,1705.0)$ & $(3.07,800.4,1617.8)$ & $(3.08,802.0,1269.0)$ & $(3.11,818.3,1007.0)$ & $(3.13,813.4,831.4)$ \\
\hline \multicolumn{6}{|c|}{$n=50$} \\
\hline 0.01 & - & - & - & - & - \\
\hline 0.02 & - & - & - & - & - \\
\hline 0.05 & - & - & - & - & - \\
\hline 0.10 & $(3.18,843.0,995.8)$ & $(3.16,837.5,907.1)$ & $(3.14,841.2,850.0)$ & $(3.13,842.4,842.2)$ & $(3.13,842.6,842.1)$ \\
\hline 0.15 & $(2.86,267.9,336.9)$ & $(2.81,259.3,302.2)$ & $(2.79,262.3,279.7)$ & $(2.78,261.4,264.9)$ & $(2.78,261.7,261.5)$ \\
\hline 0.20 & $(3.30,1292.0,1640.8)$ & $(3.27,1267.4,1503.2)$ & $(3.24,1238.4,1345.1)$ & $(3.23,1235.3,1267.0)$ & $(3.23,1236.2,1239.1)$ \\
\hline \multicolumn{6}{|c|}{$n=75$} \\
\hline 0.01 & - & - & - & - & - \\
\hline 0.02 & - & - & - & - & - \\
\hline 0.05 & $(3.20,1361.4,1412.5)$ & $(3.20,1404.2,1415.8)$ & $(3.20,1416.8,1416.5)$ & $(3.20,1417.0,1416.5)$ & $(3.20,1417.0,1416.5)$ \\
\hline 0.10 & $(2.92,501.0,1022.6)$ & $(2.98,485.6,617.6)$ & $(3.02,486.0,488.4)$ & $(3.04,486.0,485.5)$ & $(3.05,486.0,485.5)$ \\
\hline 0.15 & $(3.00,533.6,993.5)$ & $(3.01,548.4,812.6)$ & $(3.02,535.4,558.2)$ & $(3.03,534.9,534.8)$ & $(3.04,535.0,534.5)$ \\
\hline 0.20 & $(3.21,915.4,1713.5)$ & $(3.20,758.3,787.1)$ & $(3.20,805.6,808.7)$ & $(3.20,812.9,812.6)$ & $(3.20,813.5,813.0)$ \\
\hline \multicolumn{6}{|c|}{$n=100$} \\
\hline 0.01 & - & - & - & - & - \\
\hline 0.02 & - & - & - & - & - \\
\hline 0.05 & - & - & - & - & - \\
\hline 0.10 & - & - & - & - & - \\
\hline 0.15 & - & - & - & - & - \\
\hline 0.20 & - & - & - & - & - \\
\hline
\end{tabular}

Table 13: $\left(K^{\prime}, \mathrm{ARL}_{0}, \mathrm{SDRL}_{0}\right)$ for the hypergeometric $n p$ chart with $N=200, n \in\{25,50,75,100\}, p_{0} \in$ $\{0.01,0.02,0.05,0.10,0.15,0.20\}, m \in\{10,20,50,100,200\}$

\begin{tabular}{|c|c|c|c|c|c|}
\hline$p_{0}$ & $m=10$ & $m=20$ & $m=50$ & $m=100$ & $m=200$ \\
\hline \multicolumn{6}{|c|}{$n=25$} \\
\hline 0.01 & $(3.05,62.1,65.5)$ & $(3.05,64.4,65.7)$ & $(3.48,66.3,65.8)$ & $(3.48,66.3,65.8)$ & $(3.61,66.3,65.8)$ \\
\hline 0.02 & $(2.25,174.0,1054.2)$ & $(2.49,152.3,669.2)$ & $(2.73,153.7,412.8)$ & $(2.88,156.1,226.3)$ & $(2.97,155.8,157.7)$ \\
\hline 0.05 & $(2.61,306.8,2699.9)$ & $(2.80,300.8,951.4)$ & $(3.00,297.6,597.8)$ & $(3.08,295.2,379.6)$ & $(3.13,296.5,303.2)$ \\
\hline 0.10 & $(2.47,186.1,653.5)$ & $(2.62,184.0,393.9)$ & $(2.74,192.0,294.8)$ & $(2.78,188.4,218.7)$ & $(2.81,189.3,191.8)$ \\
\hline 0.15 & $(2.59,226.3,470.6)$ & $(2.66,229.5,438.2)$ & $(2.74,221.8,326.3)$ & $(2.78,222.3,257.5)$ & $(2.81,223.4,227.4)$ \\
\hline 0.20 & $(2.87,345.8,632.6)$ & $(2.86,317.4,500.3)$ & $(2.87,334.3,474.1)$ & $(2.89,333.8,394.5)$ & $(2.91,332.0,340.3)$ \\
\hline \multicolumn{6}{|c|}{$n=50$} \\
\hline 0.01 & - & - & - & - & - \\
\hline 0.02 & $(3.29,256.3,277.6)$ & $(3.38,275.4,279.9)$ & $(3.44,280.7,280.4)$ & $(3.47,280.9,280.4)$ & $(3.49,280.9,280.4)$ \\
\hline 0.05 & $(2.50,375.7,2828.9)$ & $(2.69,374.4,1251.5)$ & $(2.85,367.5,694.0)$ & $(2.91,365.1,476.0)$ & $(2.95,365.4,375.6)$ \\
\hline 0.10 & $(2.90,448.5,881.6)$ & $(2.90,422.1,764.3)$ & $(2.90,417.2,642.5)$ & $(2.93,416.2,509.9)$ & $(2.96,419.2,435.9)$ \\
\hline 0.15 & $(3.16,788.3,1252.6)$ & $(3.15,822.4,1288.0)$ & $(3.14,795.7,1086.0)$ & $(3.15,801.3,942.5)$ & $(3.17,806.5,838.4)$ \\
\hline 0.20 & $(3.08,507.9,703.8)$ & $(3.05,492.2,643.4)$ & $(3.03,501.0,596.9)$ & $(3.03,503.4,550.3)$ & $(3.04,507.2,518.6)$ \\
\hline \multicolumn{6}{|c|}{$n=75$} \\
\hline 0.01 & - & - & - & - & - \\
\hline 0.02 & - & - & - & - & - \\
\hline 0.05 & $(2.79,1195.4,5906.4)$ & $(2.85,1326.4,5574.8)$ & $(3.00,1325.7,3829.8)$ & $(3.08,1352.5,2403.0)$ & $(3.13,1359.9,1510.3)$ \\
\hline 0.10 & $(2.92,366.2,543.9)$ & $(2.90,383.6,529.4)$ & $(2.89,377.0,453.2)$ & $(2.89,372.0,400.9)$ & $(2.90,371.5,375.6)$ \\
\hline 0.15 & $(3.08,473.9,626.5)$ & $(3.05,472.4,576.5)$ & $(3.04,478.5,532.3)$ & $(3.03,465.4,469.2)$ & $(3.03,472.6,473.5)$ \\
\hline 0.20 & $(3.16,593.9,747.9)$ & $(3.13,603.4,724.3)$ & $(3.10,591.7,660.5)$ & $(3.09,591.9,627.8)$ & $(3.09,594.6,603.9)$ \\
\hline \multicolumn{6}{|c|}{$n=100$} \\
\hline 0.01 & - & - & - & - & - \\
\hline 0.02 & - & - & - & - & - \\
\hline 0.05 & $(3.12,646.7,764.8)$ & $(3.09,643.2,698.6)$ & $(3.08,649.7,659.8)$ & $(3.07,648.5,648.6)$ & $(3.06,648.5,648.0)$ \\
\hline 0.10 & $(3.12,624.1,802.3)$ & $(3.09,646.9,774.8)$ & $(3.06,647.7,708.9)$ & $(3.05,648.0,670.6)$ & $(3.05,649.6,652.4)$ \\
\hline 0.15 & $(3.04,397.4,482.8)$ & $(3.01,402.4,458.3)$ & $(2.98,402.3,433.7)$ & $(2.97,399.2,412.4)$ & $(2.96,397.8,400.1)$ \\
\hline 0.20 & $(3.07,422.9,501.3)$ & $(3.05,432.7,486.8)$ & $(3.02,425.5,454.1)$ & $(3.01,426.0,439.5)$ & $(3.00,423.1,426.3)$ \\
\hline
\end{tabular}


Table 14: $\left(K^{\prime}, \mathrm{ARL}_{0}, \mathrm{SDRL}_{0}\right)$ for the hypergeometric $n p$ chart with $N=500, n \in\{25,50,75,100\}, p_{0} \in$ $\{0.01,0.02,0.05,0.10,0.15,0.20\}, m \in\{10,20,50,100,200\}$

\begin{tabular}{|c|c|c|c|c|c|}
\hline$p_{0}$ & $m=10$ & $m=20$ & $m=50$ & $m=100$ & $m=200$ \\
\hline \multicolumn{6}{|c|}{$n=25$} \\
\hline 0.01 & $(1.85,44.8,192.8)$ & $(2.16,45.2,147.7)$ & $(2.35,46.4,92.6)$ & $(2.44,45.7,49.5)$ & $(2.50,45.7,45.2)$ \\
\hline 0.02 & $(2.29,90.4,497.8)$ & $(2.54,92.9,272.7)$ & $(2.72,95.8,165.3)$ & $(2.82,94.8,111.8)$ & $(2.86,95.0,95.2)$ \\
\hline 0.05 & $(2.53,163.7,837.5)$ & $(2.76,170.2,433.9)$ & $(2.91,180.1,290.4)$ & $(2.97,180.9,219.5)$ & $(3.01,180.1,182.8)$ \\
\hline 0.10 & $(2.40,137.1,417.4)$ & $(2.53,132.8,265.2)$ & $(2.63,130.0,184.5)$ & $(2.68,130.2,149.2)$ & $(2.70,129.7,131.1)$ \\
\hline 0.15 & $(2.50,154.7,294.8)$ & $(2.56,154.3,270.5)$ & $(2.64,152.0,211.5)$ & $(2.68,153.9,174.5)$ & $(2.70,154.1,156.5)$ \\
\hline 0.20 & $(2.76,224.9,376.8)$ & $(2.75,210.9,308.0)$ & $(2.76,225.1,305.3)$ & $(2.78,225.4,261.4)$ & $(2.79,224.0,228.7)$ \\
\hline \multicolumn{6}{|c|}{$n=50$} \\
\hline 0.01 & $(2.19,116.9,1484.3)$ & $(2.49,133.3,515.7)$ & $(2.73,123.0,284.2)$ & $(2.84,121.9,149.5)$ & $(2.92,122.4,123.4)$ \\
\hline 0.02 & $(2.18,82.0,435.8)$ & $(2.35,84.8,219.0)$ & $(2.51,84.9,131.8)$ & $(2.58,84.2,94.8)$ & $(2.62,84.3,84.4)$ \\
\hline 0.05 & $(2.38,128.9,466.4)$ & $(2.51,132.8,265.4)$ & $(2.63,127.6,184.2)$ & $(2.67,129.5,149.0)$ & $(2.70,129.7,131.8)$ \\
\hline 0.10 & $(2.91,555.6,1483.5)$ & $(3.03,561.8,1207.4)$ & $(3.13,549.3,828.3)$ & $(3.17,553.1,685.8)$ & $(3.20,556.9,590.6)$ \\
\hline 0.15 & $(2.89,277.9,385.6)$ & $(2.88,284.1,389.7)$ & $(2.88,290.5,367.7)$ & $(2.89,290.9,335.3)$ & $(2.90,288.9,300.3)$ \\
\hline 0.20 & $(3.15,614.0,848.9)$ & $(3.14,639.4,854.8)$ & $(3.14,657.6,824.9)$ & $(3.14,645.3,742.7)$ & $(3.15,646.1,679.7)$ \\
\hline \multicolumn{6}{|c|}{$n=75$} \\
\hline 0.01 & $(2.42,362.9,2363.7)$ & $(2.83,441.7,2254.5)$ & $(3.14,469.4,1550.2)$ & $(3.29,480.5,917.3)$ & $(3.37,478.4,529.9)$ \\
\hline 0.02 & $(2.20,109.2,492.2)$ & $(2.40,109.9,280.0)$ & $(2.54,108.0,171.8)$ & $(2.61,110.0,127.7)$ & $(2.64,109.5,110.3)$ \\
\hline 0.05 & $(2.49,159.6,303.5)$ & $(2.53,156.0,290.9)$ & $(2.64,155.4,221.5)$ & $(2.67,153.2,177.3)$ & $(2.70,154.3,158.3)$ \\
\hline 0.10 & $(2.89,293.1,420.6)$ & $(2.88,292.6,408.2)$ & $(2.88,294.1,375.9)$ & $(2.88,286.2,329.8)$ & $(2.90,289.1,302.3)$ \\
\hline 0.15 & $(2.95,289.4,358.3)$ & $(2.94,300.4,347.1)$ & $(2.94,302.9,325.1)$ & $(2.93,298.8,311.4)$ & $(2.94,303.4,308.2)$ \\
\hline 0.20 & $(3.01,348.2,430.6)$ & $(2.99,349.1,415.5)$ & $(2.97,353.2,399.6)$ & $(2.96,351.4,378.5)$ & $(2.96,353.7,364.5)$ \\
\hline \multicolumn{6}{|c|}{$n=100$} \\
\hline 0.01 & $(2.14,171.4,824.5)$ & $(2.47,168.6,670.5)$ & $(2.60,157.2,379.1)$ & $(2.69,155.1,210.1)$ & $(2.75,155.3,157.2)$ \\
\hline 0.02 & $(2.32,174.6,1166.0)$ & $(2.51,171.1,454.3)$ & $(2.66,174.3,298.2)$ & $(2.71,170.1,204.3)$ & $(2.75,170.9,173.2)$ \\
\hline 0.05 & $(2.74,224.0,377.3)$ & $(2.73,216.0,338.1)$ & $(2.74,226.7,325.5)$ & $(2.77,225.2,271.3)$ & $(2.79,224.4,233.3)$ \\
\hline 0.10 & $(3.14,617.7,853.3)$ & $(3.14,657.1,893.6)$ & $(3.13,650.1,832.4)$ & $(3.13,637.8,744.0)$ & $(3.14,639.7,677.6)$ \\
\hline 0.15 & $(3.01,354.3,446.7)$ & $(2.99,355.0,424.8)$ & $(2.97,355.2,403.1)$ & $(2.96,353.2,383.3)$ & $(2.96,354.5,366.5)$ \\
\hline 0.20 & $(3.00,317.8,373.3)$ & $(2.97,313.8,354.8)$ & $(2.94,310.8,338.2)$ & $(2.93,310.5,328.3)$ & $(2.93,313.9,322.5)$ \\
\hline
\end{tabular}

Table 15: $\left(K^{\prime}, \mathrm{ARL}_{0}, \mathrm{SDRL}_{0}\right)$ for the hypergeometric $n p$ chart with $N=1000, n \in\{25,50,75,100\}$, $p_{0} \in\{0.01,0.02,0.05,0.10,0.15,0.20\}, m \in\{10,20,50,100,200\}$

\begin{tabular}{|c|c|c|c|c|c|}
\hline$p_{0}$ & $m=10$ & $m=20$ & $m=50$ & $m=100$ & $m=200$ \\
\hline \multicolumn{6}{|c|}{$n=25$} \\
\hline 0.01 & $(1.85,39.4,129.4)$ & $(2.14,39.8,110.2)$ & $(2.32,42.0,72.7)$ & $(2.41,41.8,44.2)$ & $(2.47,41.9,41.4)$ \\
\hline 0.02 & $(2.26,76.8,333.1)$ & $(2.51,79.8,210.1)$ & $(2.69,84.1,134.2)$ & $(2.79,84.3,96.1)$ & $(2.83,84.1,84.1)$ \\
\hline 0.05 & $(2.51,166.8,674.0)$ & $(2.74,164.5,398.9)$ & $(2.87,156.6,242.8)$ & $(2.94,158.0,188.4)$ & $(2.97,157.5,159.7)$ \\
\hline 0.10 & $(2.37,121.6,350.6)$ & $(2.50,118.3,228.9)$ & $(2.61,116.4,162.3)$ & $(2.65,116.9,133.0)$ & $(2.67,116.6,118.1)$ \\
\hline 0.15 & $(2.90,579.7,2157.1)$ & $(3.06,555.8,1255.4)$ & $(3.16,545.3,847.8)$ & $(3.20,535.8,652.2)$ & $(3.23,539.0,561.9)$ \\
\hline 0.20 & $(2.74,198.8,325.1)$ & $(2.72,197.3,294.3)$ & $(2.72,199.0,263.9)$ & $(2.74,199.8,227.9)$ & $(2.76,200.2,204.7)$ \\
\hline \multicolumn{6}{|c|}{$n=50$} \\
\hline 0.01 & $(2.29,90.6,634.0)$ & $(2.51,87.9,248.9)$ & $(2.71,91.3,153.5)$ & $(2.78,90.6,105.8)$ & $(2.85,90.8,90.9)$ \\
\hline 0.02 & $(2.12,62.2,234.4)$ & $(2.30,69.6,145.9)$ & $(2.45,67.3,95.4)$ & $(2.51,67.3,73.8)$ & $(2.55,67.5,67.4)$ \\
\hline 0.05 & $(2.87,420.6,2109.6)$ & $(3.06,428.8,995.2)$ & $(3.20,425.0,671.6)$ & $(3.26,428.0,548.4)$ & $(3.29,424.6,447.5)$ \\
\hline 0.10 & $(2.86,406.5,1027.0)$ & $(2.95,404.9,812.4)$ & $(3.05,399.4,578.2)$ & $(3.09,403.7,490.0)$ & $(3.11,405.4,427.7)$ \\
\hline 0.15 & $(2.82,225.9,313.0)$ & $(2.81,228.9,304.7)$ & $(2.80,220.3,269.3)$ & $(2.81,223.8,253.4)$ & $(2.82,223.0,231.1)$ \\
\hline 0.20 & $(3.07,457.7,619.9)$ & $(3.06,474.2,618.8)$ & $(3.06,487.2,599.1)$ & $(3.06,485.9,556.1)$ & $(3.06,477.3,499.4)$ \\
\hline \multicolumn{6}{|c|}{$n=75$} \\
\hline 0.01 & $(2.66,224.6,2273.5)$ & $(2.89,222.9,704.1)$ & $(3.08,225.3,426.1)$ & $(3.18,228.1,299.9)$ & $(3.25,230.4,237.9)$ \\
\hline 0.02 & $(2.84,431.5,2345.5)$ & $(3.10,421.1,1202.7)$ & $(3.26,418.2,777.4)$ & $(3.33,411.8,554.6)$ & $(3.37,412.3,433.2)$ \\
\hline 0.05 & $(2.78,378.0,1373.9)$ & $(2.95,370.4,791.8)$ & $(3.07,370.3,559.9)$ & $(3.11,371.1,458.9)$ & $(3.13,367.6,387.0)$ \\
\hline 0.10 & $(3.06,568.5,942.3)$ & $(3.11,566.1,831.6)$ & $(3.14,564.6,760.5)$ & $(3.16,568.3,691.8)$ & $(3.17,559.3,599.9)$ \\
\hline 0.15 & $(3.06,462.8,646.4)$ & $(3.05,443.2,575.7)$ & $(3.06,450.2,540.9)$ & $(3.07,447.9,497.7)$ & $(3.07,446.3,464.7)$ \\
\hline 0.20 & $(3.17,601.2,774.0)$ & $(3.15,606.7,749.7)$ & $(3.14,604.3,699.8)$ & $(3.14,612.7,681.4)$ & $(3.14,611.0,642.9)$ \\
\hline \multicolumn{6}{|c|}{$n=100$} \\
\hline 0.01 & $(2.16,78.4,399.2)$ & $(2.34,81.1,205.3)$ & $(2.50,81.6,125.0)$ & $(2.56,81.0,90.9)$ & $(2.60,81.1,81.2)$ \\
\hline 0.02 & $(2.26,105.1,335.7)$ & $(2.41,93.7,184.5)$ & $(2.52,94.7,134.4)$ & $(2.57,95.8,108.9)$ & $(2.60,95.8,96.7)$ \\
\hline 0.05 & $(2.84,406.2,1027.1)$ & $(2.94,409.3,863.4)$ & $(3.05,408.3,601.0)$ & $(3.09,407.0,499.6)$ & $(3.11,406.2,432.2)$ \\
\hline 0.10 & $(2.96,323.5,429.2)$ & $(2.96,338.1,435.7)$ & $(2.95,332.5,404.0)$ & $(2.96,339.1,388.3)$ & $(2.97,336.8,356.0)$ \\
\hline 0.15 & $(3.11,496.3,637.0)$ & $(3.10,508.3,626.0)$ & $(3.09,506.1,587.3)$ & $(3.08,496.6,549.0)$ & $(3.09,505.4,533.2)$ \\
\hline 0.20 & $(3.08,413.4,499.0)$ & $(3.05,412.3,476.4)$ & $(3.03,407.8,449.1)$ & $(3.03,420.8,452.4)$ & $(3.02,413.0,427.4)$ \\
\hline
\end{tabular}


Table 16: $\left(K^{\prime}, \mathrm{ARL}_{0}, \mathrm{SDRL}_{0}\right)$ for the hypergeometric $n p$ chart with $N=2000, n \in\{25,50,75,100\}$, $p_{0} \in\{0.01,0.02,0.05,0.10,0.15,0.20\}, m \in\{10,20,50,100,200\}$

\begin{tabular}{|c|c|c|c|c|c|}
\hline$p_{0}$ & $m=10$ & $m=20$ & $m=50$ & $m=100$ & $m=200$ \\
\hline \multicolumn{6}{|c|}{$n=25$} \\
\hline 0.01 & $(1.84,37.3,112.4)$ & $(2.12,37.8,98.2)$ & $(2.31,40.3,66.2)$ & $(2.39,40.2,42.2)$ & $(2.46,40.3,39.8)$ \\
\hline 0.02 & $(2.25,71.6,285.6)$ & $(2.50,74.7,188.0)$ & $(2.68,79.3,122.7)$ & $(2.77,79.7,89.9)$ & $(2.81,79.6,79.5)$ \\
\hline 0.05 & $(2.50,154.6,596.9)$ & $(2.72,153.1,361.7)$ & $(2.86,146.8,223.8)$ & $(2.92,148.4,175.6)$ & $(2.95,148.0,150.0)$ \\
\hline 0.10 & $(2.35,109.3,278.0)$ & $(2.48,112.1,213.7)$ & $(2.59,110.6,152.7)$ & $(2.63,111.1,126.0)$ & $(2.65,110.8,111.9)$ \\
\hline 0.15 & $(2.88,486.3,1882.6)$ & $(3.04,514.5,1143.9)$ & $(3.14,506.0,778.6)$ & $(3.18,497.9,603.6)$ & $(3.21,501.1,522.2)$ \\
\hline 0.20 & $(2.72,187.4,303.1)$ & $(2.71,199.7,307.1)$ & $(2.71,189.5,251.6)$ & $(2.72,188.3,215.0)$ & $(2.74,189.4,193.4)$ \\
\hline \multicolumn{6}{|c|}{$n=50$} \\
\hline 0.01 & $(2.26,76.4,414.0)$ & $(2.47,75.7,192.6)$ & $(2.67,77.4,124.8)$ & $(2.77,80.2,91.1)$ & $(2.81,80.5,80.5)$ \\
\hline 0.02 & $(2.10,66.1,207.7)$ & $(2.27,62.4,124.2)$ & $(2.42,60.9,83.8)$ & $(2.48,61.1,66.5)$ & $(2.52,61.4,61.3)$ \\
\hline 0.05 & $(2.84,373.0,1615.0)$ & $(3.03,361.8,801.8)$ & $(3.16,361.1,554.5)$ & $(3.22,364.9,459.7)$ & $(3.25,362.7,381.3)$ \\
\hline 0.10 & $(2.82,348.8,846.3)$ & $(2.93,354.8,722.2)$ & $(3.02,359.1,512.7)$ & $(3.05,350.7,422.0)$ & $(3.07,352.5,371.4)$ \\
\hline 0.15 & $(2.78,191.0,255.0)$ & $(2.78,203.0,266.8)$ & $(2.77,200.3,246.8)$ & $(2.78,201.6,228.3)$ & $(2.79,200.1,208.0)$ \\
\hline 0.20 & $(3.03,400.0,537.1)$ & $(3.02,413.7,534.3)$ & $(3.02,424.9,518.0)$ & $(3.02,421.2,476.3)$ & $(3.02,416.8,435.1)$ \\
\hline \multicolumn{6}{|c|}{$n=75$} \\
\hline 0.01 & $(2.62,174.8,1158.0)$ & $(2.84,191.7,517.7)$ & $(3.03,179.6,327.2)$ & $(3.13,177.8,230.3)$ & $(3.19,178.7,182.8)$ \\
\hline 0.02 & $(2.82,314.4,1642.3)$ & $(3.07,312.0,793.4)$ & $(3.20,315.0,542.1)$ & $(3.27,314.2,404.8)$ & $(3.31,315.9,329.7)$ \\
\hline 0.05 & $(2.73,296.3,972.0)$ & $(2.90,294.1,597.8)$ & $(3.01,295.0,431.1)$ & $(3.05,296.9,360.5)$ & $(3.07,294.8,309.5)$ \\
\hline 0.10 & $(3.00,455.6,747.3)$ & $(3.05,456.5,668.7)$ & $(3.08,451.9,599.1)$ & $(3.09,445.1,534.2)$ & $(3.11,448.5,479.1)$ \\
\hline 0.15 & $(3.00,374.9,516.2)$ & $(2.99,360.2,461.6)$ & $(3.00,366.9,436.7)$ & $(3.01,365.2,404.1)$ & $(3.01,363.6,378.3)$ \\
\hline 0.20 & $(3.11,484.8,616.9)$ & $(3.09,489.1,597.6)$ & $(3.08,487.4,559.3)$ & $(3.08,497.0,549.0)$ & $(3.08,494.5,519.2)$ \\
\hline \multicolumn{6}{|c|}{$n=100$} \\
\hline 0.01 & $(2.10,59.6,216.6)$ & $(2.29,66.8,137.4)$ & $(2.44,64.8,90.9)$ & $(2.50,64.9,71.0)$ & $(2.53,65.1,65.0)$ \\
\hline 0.02 & $(2.80,332.1,1657.6)$ & $(3.01,319.7,787.2)$ & $(3.15,325.2,518.7)$ & $(3.22,331.6,425.1)$ & $(3.25,330.5,345.3)$ \\
\hline 0.05 & $(2.77,309.3,769.0)$ & $(2.88,299.9,595.8)$ & $(2.97,300.9,425.6)$ & $(3.01,303.9,368.6)$ & $(3.03,302.1,319.6)$ \\
\hline 0.10 & $(2.88,248.5,324.9)$ & $(2.88,258.2,326.3)$ & $(2.88,259.3,310.2)$ & $(2.88,256.9,290.1)$ & $(2.89,258.2,272.4)$ \\
\hline 0.15 & $(3.03,373.6,473.2)$ & $(3.02,382.2,463.9)$ & $(3.01,382.8,439.8)$ & $(3.00,374.4,410.7)$ & $(3.00,375.4,393.7)$ \\
\hline 0.20 & $(3.00,315.1,376.4)$ & $(2.97,314.8,359.8)$ & $(2.96,325.0,357.2)$ & $(2.95,321.4,343.5)$ & $(2.94,315.9,326.4)$ \\
\hline
\end{tabular}

Table 17: $\left(K^{\prime}, \mathrm{ARL}_{0}, \mathrm{SDRL}_{0}\right)$ for the hypergeometric $n p$ chart with $N=5000, n \in\{25,50,75,100\}$, $p_{0} \in\{0.01,0.02,0.05,0.10,0.15,0.20\}, m \in\{10,20,50,100,200\}$

\begin{tabular}{|c|c|c|c|c|c|}
\hline$p_{0}$ & $m=10$ & $m=20$ & $m=50$ & $m=100$ & $m=200$ \\
\hline \multicolumn{6}{|c|}{$n=25$} \\
\hline 0.01 & $(1.81,36.3,104.0)$ & $(2.12,36.7,92.2)$ & $(2.31,39.3,63.0)$ & $(2.38,39.3,41.1)$ & $(2.45,39.4,38.9)$ \\
\hline 0.02 & $(2.24,68.8,263.0)$ & $(2.49,71.9,176.8)$ & $(2.67,76.7,116.8)$ & $(2.76,77.2,86.6)$ & $(2.80,77.1,77.0)$ \\
\hline 0.05 & $(2.49,148.0,557.4)$ & $(2.71,147.0,342.1)$ & $(2.85,141.4,213.6)$ & $(2.91,143.1,168.7)$ & $(2.94,142.8,144.7)$ \\
\hline 0.10 & $(2.34,105.8,265.9)$ & $(2.47,108.6,205.4)$ & $(2.58,107.3,147.4)$ & $(2.62,107.8,122.1)$ & $(2.64,107.5,108.7)$ \\
\hline 0.15 & $(2.87,464.7,1769.5)$ & $(3.03,491.9,1083.7)$ & $(3.13,484.4,741.0)$ & $(3.17,480.8,590.6)$ & $(3.20,480.8,503.0)$ \\
\hline 0.20 & $(2.71,181.1,291.0)$ & $(2.70,192.9,294.9)$ & $(2.70,183.4,242.7)$ & $(2.71,182.4,208.0)$ & $(2.73,183.4,187.3)$ \\
\hline \multicolumn{6}{|c|}{$n=50$} \\
\hline 0.01 & $(2.24,70.0,338.8)$ & $(2.46,70.1,169.2)$ & $(2.66,78.0,130.5)$ & $(2.75,75.1,84.3)$ & $(2.79,75.4,75.3)$ \\
\hline 0.02 & $(2.09,61.7,185.7)$ & $(2.25,58.7,113.8)$ & $(2.40,57.7,78.1)$ & $(2.46,58.0,62.8)$ & $(2.50,58.2,58.1)$ \\
\hline 0.05 & $(2.82,337.7,1390.4)$ & $(3.02,333.5,763.5)$ & $(3.14,329.7,498.5)$ & $(3.19,330.3,405.0)$ & $(3.22,331.0,346.2)$ \\
\hline 0.10 & $(2.80,319.9,759.0)$ & $(2.91,326.8,653.6)$ & $(3.00,331.1,468.2)$ & $(3.03,328.4,395.3)$ & $(3.05,326.1,342.9)$ \\
\hline 0.15 & $(3.06,538.9,860.3)$ & $(3.10,528.5,744.2)$ & $(3.13,549.9,732.5)$ & $(3.14,542.1,652.3)$ & $(3.16,541.7,579.5)$ \\
\hline 0.20 & $(3.01,372.4,494.3)$ & $(3.00,383.6,492.7)$ & $(3.00,393.0,476.6)$ & $(3.00,392.9,446.2)$ & $(3.00,387.7,405.8)$ \\
\hline \multicolumn{6}{|c|}{$n=75$} \\
\hline 0.01 & $(2.59,147.2,819.4)$ & $(2.81,163.1,410.2)$ & $(3.04,155.9,268.2)$ & $(3.10,157.1,195.6)$ & $(3.15,156.5,159.8)$ \\
\hline 0.02 & $(2.79,265.9,1232.9)$ & $(3.03,267.1,640.6)$ & $(3.17,271.5,450.4)$ & $(3.24,274.9,344.5)$ & $(3.28,275.2,287.9)$ \\
\hline 0.05 & $(2.71,263.1,878.6)$ & $(2.88,262.5,537.4)$ & $(2.97,260.7,374.3)$ & $(3.01,258.9,309.6)$ & $(3.04,262.1,275.5)$ \\
\hline 0.10 & $(2.97,402.5,655.4)$ & $(3.02,403.6,587.2)$ & $(3.04,395.7,514.1)$ & $(3.06,398.6,476.9)$ & $(3.08,398.9,427.4)$ \\
\hline 0.15 & $(2.97,333.3,454.9)$ & $(2.96,326.8,411.0)$ & $(2.97,331.6,390.5)$ & $(2.98,330.1,364.3)$ & $(2.98,327.5,340.7)$ \\
\hline 0.20 & $(3.08,432.4,546.5)$ & $(3.06,445.2,539.7)$ & $(3.05,442.4,507.6)$ & $(3.04,432.9,474.8)$ & $(3.04,434.3,454.1)$ \\
\hline \multicolumn{6}{|c|}{$n=100$} \\
\hline 0.01 & $(2.08,61.7,185.7)$ & $(2.25,58.7,113.8)$ & $(2.40,57.7,78.1)$ & $(2.46,58.0,62.8)$ & $(2.50,58.2,58.1)$ \\
\hline 0.02 & $(2.79,268.5,1228.1)$ & $(2.98,283.8,649.9)$ & $(3.12,277.6,439.7)$ & $(3.17,275.0,344.7)$ & $(3.20,274.8,286.3)$ \\
\hline 0.05 & $(2.73,264.5,634.2)$ & $(2.84,255.3,487.4)$ & $(2.93,259.7,356.9)$ & $(2.97,260.6,311.1)$ & $(2.98,256.9,270.7)$ \\
\hline 0.10 & $(2.84,216.7,281.7)$ & $(2.84,223.2,279.2)$ & $(2.83,219.9,259.8)$ & $(2.84,225.0,253.4)$ & $(2.84,221.9,232.8)$ \\
\hline 0.15 & $(2.99,321.4,401.9)$ & $(2.97,322.8,386.8)$ & $(2.96,323.9,369.8)$ & $(2.96,326.9,359.7)$ & $(2.96,325.6,341.9)$ \\
\hline 0.20 & $(2.96,278.1,329.1)$ & $(2.93,275.2,312.9)$ & $(2.91,275.2,300.5)$ & $(2.90,272.5,289.4)$ & $(2.90,275.8,285.4)$ \\
\hline
\end{tabular}


Table 18: $\left(K^{\prime}, \mathrm{ARL}_{0}, \mathrm{SDRL}_{0}\right)$ for the hypergeometric $n p$ chart with $N=10000, n \in\{25,50,75,100\}$, $p_{0} \in\{0.01,0.02,0.05,0.10,0.15,0.20\}, m \in\{10,20,50,100,200\}$

\begin{tabular}{|c|c|c|c|c|c|}
\hline$p_{0}$ & $m=10$ & $m=20$ & $m=50$ & $m=100$ & $m=200$ \\
\hline \multicolumn{6}{|c|}{$n=25$} \\
\hline 0.01 & $(1.83,35.9,102.1)$ & $(2.11,36.4,90.4)$ & $(2.30,39.0,62.0)$ & $(2.38,39.0,40.7)$ & $(2.44,39.1,38.6)$ \\
\hline 0.02 & $(2.24,68.0,256.2)$ & $(2.48,71.1,173.3)$ & $(2.67,75.8,115.0)$ & $(2.76,76.4,85.6)$ & $(2.80,76.3,76.2)$ \\
\hline 0.05 & $(2.48,145.9,545.1)$ & $(2.71,145.0,335.9)$ & $(2.84,139.7,210.4)$ & $(2.90,141.4,166.4)$ & $(2.94,141.1,143.0)$ \\
\hline 0.10 & $(2.34,104.7,262.1)$ & $(2.47,107.5,202.7)$ & $(2.58,106.2,145.8)$ & $(2.62,106.8,120.9)$ & $(2.64,106.5,107.6)$ \\
\hline 0.15 & $(2.87,502.8,1786.3)$ & $(3.03,485.7,1064.4)$ & $(3.13,477.6,729.1)$ & $(3.17,474.1,581.8)$ & $(3.19,472.4,490.8)$ \\
\hline 0.20 & $(2.71,179.1,287.1)$ & $(2.69,178.0,261.3)$ & $(2.69,180.3,236.4)$ & $(2.71,181.4,206.2)$ & $(2.73,181.8,185.9)$ \\
\hline \multicolumn{6}{|c|}{$n=50$} \\
\hline 0.01 & $(2.21,65.3,234.9)$ & $(2.45,68.4,162.5)$ & $(2.65,73.3,109.2)$ & $(2.74,73.5,82.3)$ & $(2.78,73.8,73.8)$ \\
\hline 0.02 & $(2.08,60.4,179.2)$ & $(2.24,57.6,110.7)$ & $(2.39,56.7,76.4)$ & $(2.45,57.0,61.7)$ & $(2.49,57.3,57.1)$ \\
\hline 0.05 & $(2.81,327.1,1317.3)$ & $(3.01,323.4,734.2)$ & $(3.13,320.2,481.8)$ & $(3.19,324.2,403.7)$ & $(3.22,323.3,341.0)$ \\
\hline 0.10 & $(2.79,311.0,732.7)$ & $(2.90,318.2,632.9)$ & $(2.99,322.5,454.7)$ & $(3.02,315.5,377.3)$ & $(3.04,317.3,334.0$ \\
\hline 0.15 & $(3.05,524.3,835.9)$ & $(3.10,541.6,775.9)$ & $(3.12,532.3,703.4)$ & $(3.13,520.2,622.2)$ & $(3.15,526.0,560.7)$ \\
\hline 0.20 & $(3.00,361.1,481.8)$ & $(2.99,373.0,478.0)$ & $(2.99,383.1,463.8)$ & $(2.99,380.2,428.5)$ & $(3.00,381.7,401.0)$ \\
\hline \multicolumn{6}{|c|}{$n=75$} \\
\hline 0.01 & $(2.58,139.7,741.4)$ & $(2.80,155.3,382.4)$ & $(3.03,149.2,252.6)$ & $(3.09,150.7,186.1)$ & $(3.14,150.2,153.3)$ \\
\hline 0.02 & $(2.79,270.9,1276.8)$ & $(3.02,254.4,599.7)$ & $(3.17,266.2,426.7)$ & $(3.22,260.3,327.0)$ & $(3.27,263.2,275.1)$ \\
\hline 0.05 & $(2.70,252.4,828.2)$ & $(2.88,252.1,511.1)$ & $(2.96,250.6,357.8)$ & $(3.00,251.2,302.9)$ & $(3.03,252.7,266.5)$ \\
\hline 0.10 & $(2.96,386.7,628.1)$ & $(3.01,387.9,563.1)$ & $(3.03,381.7,496.5)$ & $(3.05,383.2,457.5)$ & $(3.07,385.0,413.0)$ \\
\hline 0.15 & $(2.96,320.8,436.8)$ & $(2.95,321.6,405.4)$ & $(2.96,319.5,375.7)$ & $(2.97,318.2,350.8)$ & $(2.97,316.0,328.7)$ \\
\hline 0.20 & $(3.07,425.4,540.6)$ & $(3.05,428.3,517.9)$ & $(3.03,413.0,468.9)$ & $(3.03,418.7,458.7)$ & $(3.03,419.3,438.6)$ \\
\hline \multicolumn{6}{|c|}{$n=100$} \\
\hline 0.01 & $(2.07,59.1,172.7)$ & & $(2.39,55.6,74.7)$ & $(2.45,56.0,60.5)$ & $(2.48,56.3,56.1)$ \\
\hline 0.02 & $(2.77,251.6,1105.5)$ & $(2.96,266.5,595.0)$ & $(3.10,261.5,409.2)$ & $(3.16,261.8,323.6)$ & $(3.19,260.0,271.9)$ \\
\hline 0.05 & $(2.72,250.3,591.1)$ & $(2.83,242.4,457.8)$ & $(2.92,246.8,337.2)$ & $(2.95,246.3,294.4)$ & $(2.97,245.8,260.1)$ \\
\hline 0.10 & $(2.83,213.0,279.8)$ & $(2.82,213.0,265.5)$ & $(2.82,212.8,250.4)$ & $(2.82,212.2,237.5)$ & $(2.83,213.1,224.1)$ \\
\hline 0.15 & $(2.98,313.1,393.1)$ & $(2.96,314.7,375.4)$ & $(2.95,311.8,354.9)$ & $(2.94,306.1,334.2)$ & $(2.94,307.2,321.4)$ \\
\hline 0.20 & $(2.94,258.8,306.7)$ & $(2.92,266.1,300.6)$ & $(2.90,267.1,291.6)$ & $(2.89,264.4,281.3)$ & $(2.88,260.1,268.2)$ \\
\hline
\end{tabular}


Table 19: $\left(K, \mathrm{ARL}_{1}, \mathrm{SDRL}_{1}\right)$ for the hypergeometric $n p$ chart and $\left(K^{\prime \prime}, \mathrm{ARL}_{1}, \mathrm{SDRL}_{1}\right)$ for the binomial $n p$ chart with $N=100, n \in\{25,50\}, p_{0} \in\{0.01,0.05,0.10,0.20\}, m \in\{10,20,50,100,200,1000, \infty\}$

\begin{tabular}{|c|c|c|c|c|c|c|c|c|}
\hline$p_{0}$ & $\tau$ & $m=10$ & $m=20$ & $m=50$ & $m=100$ & $m=200$ & $m=1000$ & $m \rightarrow \infty$ \\
\hline \multicolumn{9}{|c|}{$n=25$} \\
\hline \multirow[t]{8}{*}{0.01} & 1.1 & - & - & - & - & - & - & - \\
\hline & & - & - & - & - & - & - & - \\
\hline & 1.2 & - & - & - & - & - & - & - \\
\hline & & - & - & - & - & - & - & - \\
\hline & 1.5 & - & - & - & - & - & - & - \\
\hline & & - & - & - & - & - & - & - \\
\hline & 2.0 & - & - & - & - & - & - & - \\
\hline & & - & - & - & - & - & - & - \\
\hline 0.05 & 1.1 & $\begin{array}{c}- \\
- \\
(3.00,475.9,2088.2) \\
(3.23,254.8,1384.4) \\
(3.00,134.1,1337.0) \\
(3.23,112.5,458.7) \\
(3.00,12.6,29.9) \\
(3.23,20.6,47.2) \\
\end{array}$ & $\begin{array}{c}- \\
- \\
(3.00,260.0,1142.6) \\
(3.48,281.4,747.3) \\
(3.00,76.0,219.6) \\
(3.48,127.6,289.7) \\
(3.00,10.9,16.0) \\
(3.48,24.1,39.3) \\
\end{array}$ & $\begin{array}{c}- \\
- \\
(3.00,190.2,319.8) \\
(3.75,348.6,641.3) \\
(3.00,67.5,93.7) \\
(3.75,158.2,264.0) \\
(3.00,11.0,12.7) \\
(3.75,29.3,39.5) \\
\end{array}$ & $\begin{array}{c}- \\
- \\
(3.00,204.8,265.2) \\
(3.90,378.8,590.3) \\
(3.00,72.7,90.8) \\
(3.90,172.7,246.4) \\
(3.00,11.6,12.9) \\
(3.90,32.1,38.3) \\
\end{array}$ & $\begin{array}{c}- \\
- \\
(3.00,225.2,272.7) \\
(4.05,408.3,647.1) \\
(3.00,79.4,93.6) \\
(4.05,184.8,267.8) \\
(3.00,12.4,13.4) \\
(4.05,33.8,40.7) \\
\end{array}$ & $\begin{array}{c}- \\
- \\
(3.00,270.3,283.8) \\
(4.25,494.7,856.0) \\
(3.00,94.1,97.8) \\
(4.25,218.2,347.0) \\
(3.00,14.2,14.1) \\
(4.25,37.8,49.5) \\
\end{array}$ & $\begin{array}{c}- \\
- \\
(3.00,286.5,285.9) \\
(3.45,326.4,325.9) \\
(3.00,99.4,98.8) \\
(3.45,153.2,152.6) \\
(3.00,14.9,14.3) \\
(3.45,30.0,29.4) \\
\end{array}$ \\
\hline 0.10 & 1.1 & $\begin{array}{c}(3.00,775.9,11211.3) \\
(3.55,1329.4,8194.7) \\
(3.00,287.5,1886.4) \\
(3.55,657.1,3366.6) \\
(3.00,39.0,107.5) \\
(3.55,121.1,403.2) \\
(3.00,6.4,9.4) \\
(3.55,18.4,37.3) \\
\end{array}$ & $\begin{array}{c}(3.00,344.4,1117.3) \\
(3.39,480.7,1161.3) \\
(3.00,153.4,390.2) \\
(3.39,262.0,577.8) \\
(3.00,27.7,46.5) \\
(3.39,60.9,107.3) \\
(3.00,5.5,6.3) \\
(3.39,11.9,16.0) \\
\end{array}$ & $\begin{array}{c}(3.00,245.2,459.1) \\
(3.34,311.7,493.9) \\
(3.00,118.5,192.2) \\
(3.34,177.5,267.0) \\
(3.00,24.5,30.5) \\
(3.34,45.7,60.3) \\
(3.00,5.3,5.2) \\
(3.34,10.0,11.1) \\
\end{array}$ & $\begin{array}{c}(3.00,214.0,254.0) \\
(3.33,264.4,347.4) \\
(3.00,107.4,120.0) \\
(3.33,153.4,193.2) \\
(3.00,23.5,24.0) \\
(3.33,41.3,47.1) \\
(3.00,5.2,4.7) \\
(3.33,9.4,9.5) \\
\end{array}$ & $\begin{array}{c}(3.00,213.0,213.9) \\
(3.40,262.2,318.0) \\
(3.00,107.4,107.4) \\
(3.40,152.6,178.7) \\
(3.00,23.6,23.1) \\
(3.40,41.3,44.8) \\
(3.00,5.3,4.7) \\
(3.40,9.5,9.3) \\
\end{array}$ & $\begin{array}{c}(3.00,213.6,213.0) \\
(3.55,266.8,332.1) \\
(3.00,107.7,107.1) \\
(3.55,155.0,185.7) \\
(3.00,23.7,23.1) \\
(3.55,41.8,46.0) \\
(3.00,5.3,4.7) \\
(3.55,9.6,9.5) \\
\end{array}$ & $\begin{array}{c}(3.00,213.6,213.0) \\
(3.00,242.7,242.2) \\
(3.00,107.7,107.1) \\
(3.00,142.5,142.0) \\
(3.00,23.7,23.1) \\
(3.00,39.3,38.8) \\
(3.00,5.3,4.7) \\
(3.00,9.2,8.6) \\
\end{array}$ \\
\hline \multirow[t]{2}{*}{0.20} & 1.5 & $\begin{array}{c}(3.00,257.3,418.8) \\
(2.90,202.3,396.3) \\
(3.00,132.6,274.7) \\
(2.90,100.2,187.7) \\
(3.00,16.5,28.6) \\
(2.90,18.0,31.8) \\
(3.00,2.5,2.4) \\
(2.90,3.1,3.3) \\
\end{array}$ & $\begin{array}{c}(3.00,231.4,327.3) \\
(3.00,224.5,411.9) \\
(3.00,105.0,171.7) \\
(3.00,105.5,177.3) \\
(3.00,14.0,18.5) \\
(3.00,18.4,24.8) \\
(3.00,2.4,2.0) \\
(3.00,3.3,3.1) \\
\end{array}$ & $\begin{array}{c}(3.00,218.3,263.1) \\
(3.13,251.8,352.5) \\
(3.00,91.1,113.3) \\
(3.13,118.6,157.2) \\
(3.00,12.9,14.1) \\
(3.13,20.5,23.7) \\
(3.00,2.3,1.8) \\
(3.13,3.5,3.2) \\
\end{array}$ & $\begin{array}{c}(3.00,233.7,260.0) \\
(3.22,284.5,342.4) \\
(3.00,95.0,104.3) \\
(3.22,133.6,154.8) \\
(3.00,13.4,13.6) \\
(3.22,22.8,24.1) \\
(3.00,2.4,1.8) \\
(3.22,3.8,3.3) \\
\end{array}$ & $\begin{array}{c}(3.00,252.2,266.5) \\
(3.32,309.4,378.2) \\
(3.00,101.6,106.3) \\
(3.32,144.3,169.1) \\
(3.00,14.1,14.0) \\
(3.32,24.2,25.6) \\
(3.00,2.4,1.9) \\
(3.32,3.9,3.4) \\
\end{array}$ & $\begin{array}{c}(3.00,272.5,272.2) \\
(3.43,330.9,430.1) \\
(3.00,109.2,108.8) \\
(3.43,153.1,189.5) \\
(3.00,14.9,14.3) \\
(3.43,25.1,27.7) \\
(3.00,2.5,1.9) \\
(3.43,4.0,3.5) \\
\end{array}$ & $\begin{array}{c}(3.00,272.8,272.3) \\
(3.00,276.6,276.1) \\
(3.00,109.4,108.8) \\
(3.00,130.9,130.4) \\
(3.00,14.9,14.3) \\
(3.00,22.7,22.1) \\
(3.00,2.5,1.9) \\
(3.00,3.8,3.2) \\
\end{array}$ \\
\hline & & & & & $=50$ & & & \\
\hline \multirow[t]{7}{*}{0.01} & 1.1 & - & - & - & - & - & - & - \\
\hline & & - & - & - & - & - & - & - \\
\hline & 1.2 & - & - & - & - & - & - & - \\
\hline & 1.5 & $\begin{array}{l}- \\
-\end{array}$ & $\begin{array}{l}- \\
-\end{array}$ & $\begin{array}{l}- \\
-\end{array}$ & $\begin{array}{l}- \\
-\end{array}$ & $\begin{array}{l}- \\
-\end{array}$ & $\begin{array}{l}- \\
-\end{array}$ & $\begin{array}{l}- \\
-\end{array}$ \\
\hline & & - & - & - & - & - & - & - \\
\hline & 2.0 & - & - & - & - & - & - & - \\
\hline & & - & - & - & - & - & - & - \\
\hline \multirow[t]{8}{*}{0.05} & 1.1 & - & - & - & - & - & - & - \\
\hline & & - & - & - & - & - & - & - \\
\hline & 1.2 & - & - & - & - & - & - & - \\
\hline & & - & - & - & - & - & - & - \\
\hline & 1.5 & - & - & - & - & - & - & - \\
\hline & & - & - & - & - & - & - & - \\
\hline & 2.0 & - & - & - & - & - & - & - \\
\hline & & - & - & - & - & - & - & - \\
\hline 0.10 & 1.2 & $\begin{array}{c}(3.00,513.5,1093.9) \\
(3.01,267.6,762.3) \\
(3.00,151.5,297.4) \\
(3.01,123.7,306.4) \\
(3.00,10.9,19.2) \\
(3.01,21.4,38.2) \\
(3.00,1.8,1.4) \\
(3.01,3.9,4.4) \\
\end{array}$ & $\begin{array}{c}(3.00,477.9,999.8) \\
(3.22,306.9,615.3) \\
(3.00,133.7,263.5) \\
(3.22,143.1,259.9) \\
(3.00,9.6,12.5) \\
(3.22,24.8,35.4) \\
(3.00,1.8,1.3) \\
(3.22,4.4,4.5) \\
\end{array}$ & $\begin{array}{c}(3.00,314.4,601.4) \\
(3.36,336.1,471.2) \\
(3.00,90.4,159.9) \\
(3.36,158.1,210.6) \\
(3.00,8.2,8.9) \\
(3.36,27.6,32.4) \\
(3.00,1.7,1.1) \\
(3.36,4.8,4.5) \\
\end{array}$ & $\begin{array}{c}(3.00,257.0,352.8) \\
(3.40,352.0,420.0) \\
(3.00,75.7,97.7) \\
(3.40,166.0,192.8) \\
(3.00,7.8,7.5) \\
(3.40,29.0,31.3) \\
(3.00,1.7,1.1) \\
(3.40,4.9,4.6) \\
\end{array}$ & $\begin{array}{c}(3.00,241.8,248.2) \\
(3.39,358.6,393.2) \\
(3.00,71.8,72.8) \\
(3.39,169.3,183.5) \\
(3.00,7.7,7.1) \\
(3.39,29.6,30.8) \\
(3.00,1.7,1.1) \\
(3.39,5.0,4.6)\end{array}$ & $\begin{array}{c}(3.00,240.8,240.2) \\
(3.34,358.2,391.5) \\
(3.00,71.5,71.0) \\
(3.34,169.1,182.9) \\
(3.00,7.7,7.1) \\
(3.34,29.5,30.7) \\
(3.00,1.7,1.1) \\
(3.34,5.0,4.6) \\
\end{array}$ & $\begin{array}{c}(3.00,240.8,240.2) \\
(3.30,417.5,417.0) \\
(3.00,71.5,71.0) \\
(3.30,195.5,195.0) \\
(3.00,7.7,7.1) \\
(3.30,33.3,32.8) \\
(3.00,1.7,1.1) \\
(3.30,5.4,4.8) \\
\end{array}$ \\
\hline 0.20 & 1.5 & $\begin{array}{c}(3.00,211.8,358.9) \\
(3.00,190.4,307.2) \\
(3.00,58.7,109.7) \\
(3.00,80.7,144.1) \\
(3.00,3.6,3.9) \\
(3.00,9.0,12.8) \\
(3.00,1.1,0.3) \\
(3.00,1.6,1.1)\end{array}$ & $\begin{array}{c}(3.00,180.1,275.2) \\
(2.99,159.2,231.9) \\
(3.00,46.3,72.5) \\
(2.99,63.7,93.9) \\
(3.00,3.4,3.2) \\
(2.99,7.8,9.0) \\
(3.00,1.1,0.3) \\
(2.99,1.6,1.0)\end{array}$ & $\begin{array}{c}(3.00,167.0,212.6) \\
(3.01,151.5,188.7) \\
(3.00,41.4,50.7) \\
(3.01,59.1,71.0) \\
(3.00,3.3,2.9) \\
(3.01,7.6,7.7) \\
(3.00,1.1,0.3) \\
(3.01,1.6,0.9)\end{array}$ & $\begin{array}{c}(3.00,168.3,210.3) \\
(3.06,160.5,189.4) \\
(3.00,41.4,49.1) \\
(3.06,62.0,70.4) \\
(3.00,3.3,2.9) \\
(3.06,7.9,7.8) \\
(3.00,1.1,0.3) \\
(3.06,1.6,0.9)\end{array}$ & $\begin{array}{c}(3.00,172.5,214.8) \\
(3.11,172.0,202.0) \\
(3.00,41.9,49.5) \\
(3.11,65.9,74.6) \\
(3.00,3.3,2.9) \\
(3.11,8.2,8.1) \\
(3.00,1.1,0.3) \\
(3.11,1.6,1.0)\end{array}$ & $\begin{array}{c}(3.00,195.6,235.4) \\
(3.19,237.3,269.1) \\
(3.00,45.4,52.0) \\
(3.19,87.4,96.8) \\
(3.00,3.4,3.0) \\
(3.19,9.8,9.8) \\
(3.00,1.1,0.3) \\
(3.19,1.7,1.1)\end{array}$ & $\begin{array}{c}(3.00,278.5,277.9) \\
(3.19,318.8,318.3) \\
(3.00,59.2,58.7) \\
(3.19,113.6,113.1) \\
(3.00,3.9,3.4) \\
(3.19,11.8,11.3) \\
(3.00,1.1,0.3) \\
(3.19,1.9,1.2)\end{array}$ \\
\hline
\end{tabular}


Table 20: $\left(K, \mathrm{ARL}_{1}, \mathrm{SDRL}_{1}\right)$ for the hypergeometric $n p$ chart and $\left(K^{\prime \prime}, \mathrm{ARL}_{1}, \mathrm{SDRL}_{1}\right)$ for the binomial $n p$ chart with $N=200, n \in\{25,50\}, p_{0} \in\{0.01,0.05,0.10,0.20\}, m \in\{10,20,50,100,200,1000, \infty\}$

\begin{tabular}{|c|c|c|c|c|c|c|c|c|}
\hline$p_{0}$ & $\tau$ & $m=10$ & $m=20$ & $m=50$ & $m=100$ & $m=200$ & $m=1000$ & $m \rightarrow \infty$ \\
\hline \multicolumn{9}{|c|}{$n=25$} \\
\hline \multirow[t]{8}{*}{0.01} & 1.1 & - & - & - & - & - & - & - \\
\hline & & _- & _- & - & _- & - & _- & - \\
\hline & 1.2 & _- & - & - & - & - & - & - \\
\hline & & _- & - & - & - & - & - & - \\
\hline & 1.5 & $(3.00,142.1,357.2)$ & $(3.00,145.7,356.5)$ & $(3.00,61.7,206.0)$ & $(3.00,37.3,124.8)$ & $(3.00,25.3,44.2)$ & $(3.00,24.0,23.5)$ & $(3.00,24.0,23.5)$ \\
\hline & & $(2.04,20.4,52.2)$ & $(2.34,26.6,59.3)$ & $(2.75,25.9,52.2)$ & $(2.92,25.8,51.3)$ & $(3.08,26.7,53.8)$ & $(3.31,27.6,56.6)$ & $(3.00,18.7,18.1)$ \\
\hline & 2.0 & $(3.00,83.5,651.5)$ & $(3.00,45.2,139.5)$ & $(3.00,22.8,56.0)$ & $(3.00,16.5,35.0)$ & $(3.00,13.4,16.0)$ & $(3.00,13.0,12.5)$ & $(3.00,13.0,12.5)$ \\
\hline & & $(2.04,11.5,23.5)$ & $(2.34,14.6,27.4)$ & $(2.75,14.5,24.4)$ & $(2.92,14.5,24.0)$ & $(3.08,14.8,25.1)$ & $(3.31,15.3,26.3)$ & $(3.00,11.3,10.8)$ \\
\hline \multirow[t]{8}{*}{0.05} & 1.1 & $(3.00,556.5,5892.6)$ & $(3.00,250.7,812.0)$ & $(3.00,173.5,309.3)$ & $(3.00,167.2,186.1)$ & $(3.00,173.7,175.9)$ & $(3.00,177.0,176.5)$ & $(3.00,177.0,176.5)$ \\
\hline & & $(3.39,694.0,5069.6)$ & $(3.25,295.4,756.7)$ & $(3.20,182.1,307.9)$ & $(3.26,183.2,301.4)$ & $(3.32,187.6,307.1)$ & $(3.38,181.6,299.0)$ & $(3.00,94.4,93.8)$ \\
\hline & 1.2 & $(3.00,292.1,2088.0)$ & $(3.00,148.0,413.3)$ & $(3.00,109.1,176.8)$ & $(3.00,106.8,116.4)$ & $(3.00,110.9,112.1)$ & $(3.00,113.0,112.5)$ & $(3.00,113.0,112.5)$ \\
\hline & & $(3.39,413.7,2564.6)$ & $(3.25,190.2,452.2)$ & $(3.20,121.8,198.0)$ & $(3.26,122.6,194.5)$ & $(3.32,125.4,198.1)$ & $(3.38,121.6,192.9)$ & $(3.00,66.5,65.9)$ \\
\hline & 1.5 & $(3.00,69.4,267.3)$ & $(3.00,44.3,91.0)$ & $(3.00,36.8,49.4)$ & $(3.00,37.1,38.8)$ & $(3.00,38.5,38.4)$ & $(3.00,39.1,38.6)$ & $(3.00,39.1,38.6)$ \\
\hline & & $(3.39,117.9,494.0)$ & $(3.25,64.9,127.9)$ & $(3.20,45.5,66.6)$ & $(3.26,45.8,65.8)$ & $(3.32,46.7,67.0)$ & $(3.38,45.5,65.3)$ & $(3.00,28.1,27.6)$ \\
\hline & 2.0 & $(3.00,15.6,35.3)$ & $(3.00,12.2,18.2)$ & $(3.00,11.2,12.5)$ & $(3.00,11.4,11.3)$ & $(3.00,11.8,11.3)$ & $(3.00,12.0,11.4)$ & $(3.00,12.0,11.4)$ \\
\hline & & $(3.39,27.7,73.4)$ & $(3.25,18.5,28.9)$ & $(3.20,14.4,18.2)$ & $(3.26,14.5,18.1)$ & $(3.32,14.7,18.4)$ & $(3.38,14.4,18.0)$ & $(3.00,10.3,9.7)$ \\
\hline \multirow[t]{8}{*}{0.10} & 1.1 & $(3.00,421.9,2299.3)$ & $(3.00,237.1,552.1)$ & $(3.00,182.3,298.3)$ & $(3.00,152.5,243.5)$ & $(3.00,124.2,178.9)$ & $(3.00,102.9,102.6)$ & $(3.00,102.8,102.3)$ \\
\hline & & $(3.18,455.1,2112.4)$ & $(3.13,253.3,555.2)$ & $(3.12,197.8,288.3)$ & $(3.03,164.8,207.4)$ & $(2.97,134.7,182.4)$ & $(2.96,106.9,151.4)$ & $(2.99,64.1,63.5)$ \\
\hline & 1.2 & $(3.00,204.7,878.8)$ & $(3.00,125.7,259.4)$ & $(3.00,100.2,154.2)$ & $(3.00,85.3,127.3)$ & $(3.00,71.2,95.7)$ & $(3.00,60.6,60.2)$ & $(3.00,60.6,60.0)$ \\
\hline & & $(3.18,242.1,957.8)$ & $(3.13,144.5,289.4)$ & $(3.12,116.3,161.7)$ & $(3.03,98.3,121.2)$ & $(2.97,81.3,106.9)$ & $(2.96,65.5,89.2)$ & $(2.99,41.3,40.7)$ \\
\hline & 1.5 & $(3.00,39.9,103.0)$ & $(3.00,29.3,46.4)$ & $(3.00,25.2,33.2)$ & $(3.00,22.4,28.3)$ & $(3.00,19.7,22.7)$ & $(3.00,17.7,17.2)$ & $(3.00,17.7,17.2)$ \\
\hline & & $(3.18,53.9,145.7)$ & $(3.13,37.7,60.7)$ & $(3.12,32.5,40.3)$ & $(3.03,28.5,33.0)$ & $(2.97,24.3,29.3)$ & $(2.96,20.4,24.9)$ & $(2.99,14.4,13.9)$ \\
\hline & 2.0 & $(3.00,7.6,11.6)$ & $(3.00,6.5,7.6)$ & $(3.00,6.0,6.3)$ & $(3.00,5.6,5.7)$ & $(3.00,5.2,4.9)$ & $(3.00,4.9,4.3)$ & $(3.00,4.9,4.3)$ \\
\hline & & $(3.18,10.4,17.9)$ & $(3.13,8.5,10.6)$ & $(3.12,7.9,8.4)$ & $(3.03,7.2,7.4)$ & $(2.97,6.4,6.7)$ & $(2.96,5.7,5.8)$ & $(2.99,4.6,4.0)$ \\
\hline \multirow[t]{8}{*}{0.20} & 1.1 & $(3.00,285.7,582.1)$ & $(3.00,232.2,394.3)$ & $(3.00,195.6,288.1)$ & $(3.00,169.2,229.1)$ & $(3.00,147.9,171.5)$ & $(3.00,138.2,137.7)$ & $(3.00,138.2,137.7)$ \\
\hline & & $(3.00,287.7,699.2)$ & $(3.04,256.9,485.9)$ & $(3.06,216.8,293.3)$ & $(3.00,183.0,227.7)$ & $(2.97,158.5,205.2)$ & $(2.98,149.1,195.4)$ & $(2.99,85.9,85.3)$ \\
\hline & 1.2 & $(3.00,135.0,268.5)$ & $(3.00,103.8,170.5)$ & $(3.00,87.6,120.8)$ & $(3.00,77.4,98.1)$ & $(3.00,69.1,76.7)$ & $(3.00,65.3,64.7)$ & $(3.00,65.3,64.7)$ \\
\hline & & $(3.00,132.4,288.9)$ & $(3.04,119.0,206.1)$ & $(3.06,103.5,133.5)$ & $(3.00,88.8,107.4)$ & $(2.97,77.8,97.1)$ & $(2.98,73.5,92.7)$ & $(2.99,45.1,44.6)$ \\
\hline & 1.5 & $(3.00,19.9,36.3)$ & $(3.00,16.1,22.0)$ & $(3.00,14.4,16.7)$ & $(3.00,13.4,14.5)$ & $(3.00,12.5,12.5)$ & $(3.00,12.1,11.5)$ & $(3.00,12.1,11.5)$ \\
\hline & & $(3.00,21.6,39.5)$ & $(3.04,20.0,27.7)$ & $(3.06,18.5,21.1)$ & $(3.00,16.6,18.3)$ & $(2.97,15.0,16.7)$ & $(2.98,14.4,16.1)$ & $(2.99,10.3,9.7)$ \\
\hline & 2.0 & $(3.00,3.0,3.2)$ & $(3.00,2.8,2.5)$ & $(3.00,2.7,2.2)$ & $(3.00,2.6,2.1)$ & $(3.00,2.5,1.9)$ & $(3.00,2.5,1.9)$ & $(3.00,2.5,1.9)$ \\
\hline & & $(3.00,3.5,3.8)$ & $(3.04,3.4,3.3)$ & $(3.06,3.3,2.9)$ & $(3.00,3.1,2.7)$ & $(2.97,3.0,2.5)$ & $(2.98,2.9,2.5)$ & $(2.99,2.5,1.8)$ \\
\hline
\end{tabular}

\begin{tabular}{|c|c|c|c|c|c|c|c|c|}
\hline \multirow[t]{8}{*}{0.01} & \multirow[t]{2}{*}{1.1} & - & - & - & - & - & - & - \\
\hline & & - & - & - & - & - & - & - \\
\hline & \multirow[t]{2}{*}{1.2} & - & - & - & - & - & - & - \\
\hline & & - & - & - & - & - & - & - \\
\hline & \multirow[t]{2}{*}{1.5} & - & - & - & - & - & - & - \\
\hline & & - & - & - & - & - & - & - \\
\hline & \multirow[t]{2}{*}{2.0} & - & - & - & - & - & - & - \\
\hline & & - & - & - & - & - & - & - \\
\hline \multirow[t]{8}{*}{0.05} & \multirow[t]{2}{*}{1.1} & $(3.00,760.3,14481.2)$ & $(3.00,350.0,1106.4)$ & $(3.00,236.6,460.3)$ & $(3.00,184.1,276.9)$ & $(3.00,166.8,177.4)$ & $(3.00,165.1,164.6)$ & $(3.00,165.1,164.6)$ \\
\hline & & $(3.64,1288.9,8690.3)$ & $(3.50,493.6,1194.3)$ & $(3.41,312.6,476.8)$ & $(3.33,232.6,326.1)$ & $(3.35,205.8,263.6)$ & $(3.46,198.9,245.7)$ & $(3.00,176.9,176.4)$ \\
\hline & \multirow[t]{2}{*}{1.2} & $(3.00,277.2,2171.7)$ & $(3.00,154.2,381.8)$ & $(3.00,113.1,193.8)$ & $(3.00,92.9,124.0)$ & $(3.00,86.2,89.1)$ & $(3.00,85.5,85.0)$ & $(3.00,85.5,85.0)$ \\
\hline & & $(3.64,638.4,3487.8)$ & $(3.50,269.4,594.0)$ & $(3.41,178.7,260.7)$ & $(3.33,136.3,182.9)$ & $(3.35,122.1,150.3)$ & $(3.46,118.4,141.1)$ & $(3.00,106.7,106.1)$ \\
\hline & \multirow[t]{2}{*}{1.5} & $(3.00,37.9,98.9)$ & $(3.00,27.8,46.1)$ & $(3.00,23.5,30.9)$ & $(3.00,21.1,23.2)$ & $(3.00,20.3,20.0)$ & $(3.00,20.2,19.7)$ & $(3.00,20.2,19.7)$ \\
\hline & & $(3.64,119.9,403.5)$ & $(3.50,63.3,111.6)$ & $(3.41,46.8,61.0)$ & $(3.33,37.9,45.7)$ & $(3.35,35.0,39.3)$ & $(3.46,34.2,37.5)$ & $(3.00,31.7,31.1)$ \\
\hline & \multirow[t]{2}{*}{2.0} & $(3.00,6.5,9.4)$ & $(3.00,5.7,6.6)$ & $(3.00,5.3,5.3)$ & $(3.00,5.1,4.7)$ & $(3.00,5.0,4.4)$ & $(3.00,5.0,4.4)$ & $(3.00,5.0,4.4)$ \\
\hline & & $(3.64,19.0,38.2)$ & $(3.50,12.8,17.2)$ & $(3.41,10.6,11.8)$ & $(3.33,9.2,9.6)$ & $(3.35,8.8,8.7)$ & $(3.46,8.6,8.5)$ & $(3.00,8.2,7.7)$ \\
\hline \multirow[t]{8}{*}{0.10} & \multirow[t]{2}{*}{1.1} & $(3.00,250.2,417.6)$ & $(3.00,232.4,381.1)$ & $(3.00,193.4,289.3)$ & $(3.00,169.2,216.3)$ & $(3.00,156.4,165.8)$ & $(3.00,153.6,153.0)$ & $(3.00,153.6,153.0)$ \\
\hline & & $(3.04,279.8,900.5)$ & $(3.19,291.5,565.3)$ & $(3.22,243.5,334.2)$ & $(3.19,210.5,269.5)$ & $(3.19,188.5,236.1)$ & $(3.25,189.0,236.8)$ & $(3.00,144.1,143.5)$ \\
\hline & \multirow[t]{2}{*}{1.2} & $(3.00,114.0,219.3)$ & $(3.00,95.6,154.5)$ & $(3.00,80.2,109.8)$ & $(3.00,72.2,85.8)$ & $(3.00,68.0,70.2)$ & $(3.00,67.0,66.5)$ & $(3.00,67.0,66.5)$ \\
\hline & & $(3.04,128.1,353.0)$ & $(3.19,136.7,241.0)$ & $(3.22,117.8,154.1)$ & $(3.19,103.4,127.4)$ & $(3.19,93.7,112.8)$ & $(3.25,94.0,113.1)$ & $(3.00,74.0,73.4)$ \\
\hline & \multirow[t]{2}{*}{1.5} & $(3.00,15.9,27.9)$ & $(3.00,13.8,18.2)$ & $(3.00,12.5,14.0)$ & $(3.00,11.9,12.2)$ & $(3.00,11.5,11.1)$ & $(3.00,11.4,10.9)$ & $(3.00,11.4,10.9)$ \\
\hline & & $(3.04,21.8,41.3)$ & $(3.19,24.0,33.6)$ & $(3.22,22.1,25.6)$ & $(3.19,20.2,22.3)$ & $(3.19,18.8,20.4)$ & $(3.25,18.8,20.4)$ & $(3.00,16.0,15.4)$ \\
\hline & \multirow[t]{2}{*}{2.0} & $(3.00,2.7,2.7)$ & $(3.00,2.6,2.3)$ & $(3.00,2.6,2.1)$ & $(3.00,2.5,1.9)$ & $(3.00,2.5,1.9)$ & $(3.00,2.5,1.9)$ & $(3.00,2.5,1.9)$ \\
\hline & & $(3.04,3.9,4.5)$ & $(3.19,4.3,4.4)$ & $(3.22,4.2,3.9)$ & $(3.19,4.0,3.6)$ & $(3.19,3.8,3.4)$ & $(3.25,3.8,3.4)$ & $(3.00,3.5,2.9)$ \\
\hline \multirow[t]{8}{*}{0.20} & \multirow[t]{2}{*}{1.1} & $(3.00,192.9,313.4)$ & $(3.00,168.7,241.6)$ & $(3.00,151.3,183.6)$ & $(3.00,146.8,159.7)$ & $(3.00,149.7,152.6)$ & $(3.00,153.9,153.3)$ & $(3.00,153.9,153.3)$ \\
\hline & & $(3.00,190.4,307.2)$ & $(3.01,169.0,246.9)$ & $(3.03,159.0,198.8)$ & $(3.07,166.1,197.0)$ & $(3.11,172.0,202.0)$ & $(3.15,172.6,202.7)$ & $(3.00,131.7,131.1)$ \\
\hline & \multirow[t]{2}{*}{1.2} & $(3.00,72.4,132.2)$ & $(3.00,58.7,88.0)$ & $(3.00,51.1,61.8)$ & $(3.00,49.0,52.8)$ & $(3.00,49.6,50.0)$ & $(3.00,50.8,50.2)$ & $(3.00,50.8,50.2)$ \\
\hline & & $(3.00,80.7,144.1)$ & $(3.01,67.2,99.8)$ & $(3.03,61.6,74.4)$ & $(3.07,63.9,72.9)$ & $(3.11,65.9,74.6)$ & $(3.15,66.1,74.8)$ & $(3.00,52.4,51.8)$ \\
\hline & \multirow[t]{2}{*}{1.5} & $(3.00,6.2,8.1)$ & $(3.00,5.6,6.1)$ & $(3.00,5.3,5.2)$ & $(3.00,5.3,4.9)$ & $(3.00,5.3,4.8)$ & $(3.00,5.4,4.9)$ & $(3.00,5.4,4.9)$ \\
\hline & & $(3.00,9.0,12.8)$ & $(3.01,8.1,9.3)$ & $(3.03,7.8,8.0)$ & $(3.07,8.0,8.0)$ & $(3.11,8.2,8.1)$ & $(3.15,8.2,8.1)$ & $(3.00,7.2,6.6)$ \\
\hline & \multirow[t]{2}{*}{2.0} & $(3.00,1.3,0.6)$ & $(3.00,1.3,0.6)$ & $(3.00,1.3,0.6)$ & $(3.00,1.3,0.6)$ & $(3.00,1.3,0.6)$ & $(3.00,1.3,0.6)$ & $(3.00,1.3,0.6)$ \\
\hline & & $(3.00,1.6,1.1)$ & $(3.01,1.6,1.0)$ & $(3.03,1.6,0.9)$ & $(3.07,1.6,1.0)$ & $(3.11,1.6,1.0)$ & $(3.15,1.6,1.0)$ & $(3.00,1.6,0.9)$ \\
\hline
\end{tabular}


Table 21: $\left(K, \mathrm{ARL}_{1}, \mathrm{SDRL}_{1}\right)$ for the hypergeometric $n p$ chart and $\left(K^{\prime \prime}, \mathrm{ARL}_{1}, \mathrm{SDRL}_{1}\right)$ for the binomial $n p$ chart with $N=500, n \in\{25,50\}, p_{0} \in\{0.01,0.05,0.10,0.20\}, m \in\{10,20,50,100,200,1000, \infty\}$

\begin{tabular}{|c|c|c|c|c|c|c|c|c|}
\hline$p_{0}$ & $\tau$ & $m=10$ & $m=20$ & $m=50$ & $m=100$ & $m=200$ & $m=1000$ & $m \rightarrow \infty$ \\
\hline \multicolumn{9}{|c|}{$n=25$} \\
\hline \multirow[t]{8}{*}{0.01} & 1.1 & - & - & - & - & - & - & - \\
\hline & & - & - & - & - & _- & _- & _- \\
\hline & 1.2 & $(3.00,321.4,8294.7)$ & $(3.00,145.3,529.2)$ & $(3.00,89.1,235.2)$ & $(3.00,62.0,174.4)$ & $(3.00,38.2,87.4)$ & $(3.00,31.5,30.9)$ & $(3.00,31.5,30.9)$ \\
\hline & & $3.34,429.7,6442.3)$ & $(3.34,157.1,593.8)$ & $(3.13,104.2,204.9)$ & $(3.10,65.6,149.8)$ & $(3.04,39.4,87.8)$ & $(3.23,31.6,54.8)$ & $(3.00,27.8,27.3)$ \\
\hline & 1.5 & $(3.00,162.5,2178.2)$ & $(3.00,88.3,262.8)$ & $(3.00,56.7,138.5)$ & $(3.00,40.9,103.1)$ & $(3.00,27.1,53.0)$ & $(3.00,23.2,22.6)$ & $(3.00,23.2,22.6)$ \\
\hline & & $(3.34,245.9,2871.9)$ & $(3.34,101.6,329.9)$ & $(3.13,70.0,132.7)$ & $(3.10,45.3,97.3)$ & $(3.04,28.5,57.6)$ & $3.23,23.5,36.9)$ & $(3.00,21.1,20.5)$ \\
\hline & 2.0 & $(3.00,44.1,248.4)$ & $(3.00,31.4,67.9)$ & $(3.00,22.1,44.4)$ & $(3.00,17.3,33.6)$ & $(3.00,13.1,18.8)$ & & $(3.00,11.9,11.3)$ \\
\hline & & $(3.34,73.5,489.5)$ & $(3.34,38.9,92.3)$ & $(3.13,28.9,49.6)$ & $(3.10,20.0,36.7)$ & $(3.04,14.0,22.6)$ & $(3.23,12.2,15.6)$ & $(3.00,11.3,10.8)$ \\
\hline \multirow[t]{8}{*}{0.05} & 1.1 & $(3.00,315.5,2175.2)$ & $(3.00,196.5,505.5)$ & $(3.00,149.0,256.3)$ & $(3.00,129.2,155.8)$ & $(3.00,126.9,128.0)$ & $(3.00,127.1,126.6)$ & $(3.00,127.1,126.6)$ \\
\hline & & $08,318.6,2008.2)$ & $04,207.3,49$ & $.00,103.0,20$ & $(3.10,131.7,207.6)$ & $(3.19,131.3,20$ & $0.02,120.0$ & \\
\hline & 1.2 & $(3.00,172.2,928.0)$ & $.00,115.2,265.3)$ & $(3.00,91.1,145.0)$ & $(3.00,80.8,93.5)$ & $(3.00,79.8,80.0)$ & $(3.00,80.0,79.4)$ & $(3.00,80.0,79.4)$ \\
\hline & & $(3.08,182.2,928.8)$ & $(3.04,125.3,272.3)$ & $(3.06,95.6,156.0)$ & $(3.10,83.6,124.2)$ & $(3.19,83.4,122.9)$ & & \\
\hline & 1.5 & $(3.00,56.9,198.7)$ & $(3.00,42.7,80.5)$ & $(3.00,36.3,50.2)$ & $(3.00,33.5,36.2)$ & $(3.00,33.3,33.0)$ & $(3.00,33.4,32.8)$ & $(3.00,33.4,32.8)$ \\
\hline & & $(3.08,63.9,220.3)$ & $(3.04,48.3,89.1)$ & $(3.06,39.2,57.1)$ & $(3.10,35.4,46.7)$ & $(3.19,35.3,46.3)$ & $(3.32,34.1,43.3)$ & $(3.00,29.6,29.0)$ \\
\hline & 2.0 & $(3.00,14.5,30.3)$ & $(3.00,12.3,17.8)$ & $(3.00,11.3,13.0)$ & $(3.00,10.8,10.7)$ & $(3.00,10.8,10.3)$ & $(3.00,10.8,10.3)$ & $(3.00,10.8,10.3)$ \\
\hline & & $(3.08,16.7,35.7)$ & $(3.04,14.1,20.7)$ & $(3.06,12.3,15.2)$ & $(3.10,11.5,13.0)$ & $(3.19,11.5,13.0)$ & $(3.32,11.3,12.3)$ & $(3.00,10.3,9.7)$ \\
\hline \multirow[t]{8}{*}{0.10} & 1.1 & $(3.00,324.0,1367.3)$ & $(3.00,209.2,462.2)$ & $(3.00,164.4,241.7)$ & $(3.00,154.3,221.6)$ & $(3.00,141.2,206.6)$ & $(3.00,98.0,138.5)$ & $(3.00,75.8,75.3)$ \\
\hline & & $07,315.4,13$ & $.07,221.5,455.4)$ & $(3.04,168.7,237.6)$ & $(3.02,160.0,203.9)$ & $(2.99,147.9,1$ & $(2.95,97.1$ & $(2.99,64.1,6$ \\
\hline & 1.2 & $(3.00,171.5,612.8)$ & $3.00,117.4,236.8)$ & $(3.00,95.3,134.4)$ & $(3.00,89.9,124.5)$ & $(3.00,82.8,116.3)$ & $(3.00,59.4,79.5)$ & $(3.00,47.4,46.8)$ \\
\hline & & $(3.07,172.9,632.5)$ & $(3.07,127.6,241.8)$ & $(3.04,100.2,135.7)$ & $(3.02,95.6,119.2)$ & $(2.99,88.8,11$ & $(2.95,60.0$ & $(2.99,41.3,40.7)$ \\
\hline & 1.5 & $(3.00,38.7,93.9)$ & $(3.00,30.1,48.7)$ & $(3.00,26.3,33.2)$ & $(3.00,25.1,31.3)$ & $(3.00,23.5,29.5)$ & $(3.00,18.3,2$ & $(3.00,15.6,15.0)$ \\
\hline & & $(3.07,41.4,102.9)$ & $(3.07,34.1,53.0)$ & $(3.04,28.8,35.2)$ & $(3.02,27.8,3$ & $(2.99,26.1,31.0)$ & $(2.95,19.0$ & $(2.99,14.4,13.9)$ \\
\hline & 2.0 & $.00,8.0,1$ & $3.00,7.0,8.5)$ & $(3.00,6.5,6.9)$ & $(3.00,6.3,6.7)$ & $(3.00,6.1,6.3)$ & $(3.00,5.2,5.0)$ & $(3.00,4.7,4.1)$ \\
\hline & & $(3.07,8.7,13.9)$ & $(3.07,8.0,9.7)$ & $(3.04,7.2,7.6)$ & $(3.02,7.1,7.3)$ & $(2.99,6.8,7.0)$ & $(2.95,5.5,5.5)$ & $(2.99,4.6,4.0)$ \\
\hline \multirow[t]{8}{*}{0.20} & 1.1 & $(3.00,265.7,553.9)$ & $(3.00,224.0,412.4)$ & $(3.00,189.0,264.7)$ & $(3.00,175.7,238.4)$ & $(3.00,159.2,217.5)$ & $(3.00,114.6,137.8)$ & $(3.00,102.1,1$ \\
\hline & & 264.1 & & $(3.02,195.7,2$ & $(3.00,183.0,2$ & $(2.98,166.3,2$ & $(2.96,122.1$, & $(2.99,85.9$, \\
\hline & 1.2 & $00,123.6,2$ & $(3.00,102.5,173.6)$ & $(3.00,88.8,118$ & $(3.00,83.2,10$ & $(3.00,76.1,98.9)$ & $(3.00,57.0$, & $(3.00,51.6,51.1)$ \\
\hline & & $(2.99,123.8,2$ & $(3.01,109.6,188.3)$ & $(3.02,94.2,1$ & $(3.00,88.8,1$ & $(2.98,81.3,100.5)$ & $(2.96,61.4$, & $(2.99,45.1,44.6)$ \\
\hline & 1.5 & $00,20.0,3$ & $0,17.1,2$ & $(3.00,15.7$ & $(3.00,15.0$ & $(3.00,14.1,1$ & $(3.00,11.6$ & $(3.00,10.9,10.3)$ \\
\hline & & 20.8 & $9)$ & $(3.02,17.3$ & $(3.00,16.6$, & $(2.98,15.5,1$ & $(2.96,12.6$ & $(2.99,10.3,9.7)$ \\
\hline & 2.0 & $00,3.2,3$. & $00,3.0,2$ & $0,2.9$ & $(3.00,2.9$ & $(3.00,2.8$ & $(3.00,2$. & $(3.00,2.5,1.9)$ \\
\hline & & $(2.99,3.4,3.8)$ & $(3.01,3.3,3.2)$ & $(3.02,3.2,2.8)$ & $(3.00,3.1,2.7)$ & $(2.98,3.0,2.6)$ & $(2.96,2.7,2.2)$ & $(2.99,2.5,1.8)$ \\
\hline \multicolumn{9}{|c|}{$n=50$} \\
\hline \multirow[t]{8}{*}{0.01} & 1.1 & - & - & - & - & - & - & - \\
\hline & & - & - & - & - & - & - & - \\
\hline & 1.2 & $(3.00,443.5,7915.9)$ & $(3.00,163.9,673.4)$ & $(3.00,95.3,243.8)$ & $(3.00,70.1,111.2)$ & $(3.00,66.0,67.0)$ & $(3.00,65.9,65.3)$ & $(3.00,65.9,65.3)$ \\
\hline & & ( & 4) & $(3.26,111$. & $(3.16$ & $(3.25,70.0,132.7)$ & $(3.41,69.1$, & $(3.00,44.9$, \\
\hline & 1.5 & $1.4)$ & $(3.00,82.8,248.2)$ & $(3.00,53.3,114.4)$ & $(3.00,42.3$, & $(3.0$ & $(3.00,40.5$, & $(3.00,40.5$ \\
\hline & & $1,362.9,2907.7)$ & $(3.44,113.2,307.1)$ & $(3.26,67.6,118.1)$ & $(3.16,48.6,8$ & $(3.25$ & $(3.41$ & $(3.00,30.3$, \\
\hline & 2.0 & $(3.00$ & $(3$ & $(3.0$ & $(3.00,15.0,1$ & $14.7,1$ & $(3.00,14$. & $(3.00,14.7,14.1)$ \\
\hline & & & $(3.44,34.0,67.7)$ & $(3.20,23.0,34.8)$ & $(3.16,17.8,26.2)$ & $(3.25,16.7,23.9)$ & $(3.41,16.6,23.6)$ & $(3.00,12.8,12.2)$ \\
\hline \multirow[t]{8}{*}{0.05} & 1.1 & $(3.00,360.9,1888.0)$ & $(3.00,230.3,528.1)$ & $(3.00,190.1,278.3)$ & $(3.00,175.4,250.3)$ & $(3.00,158.7,231.9)$ & $(3.00,108.7,155$ & $(3.00,82.4,81$. \\
\hline & & & & 7) & $(3.14$, & $(3.03,167.7$ & $(2.91$, & $(2.91,57.7$ \\
\hline & 1.2 & ( & $(:$ & $(3.00$ & $(3.00,88.4,120$ & $(3.00,80.7,11]$ & $(3.00,57$. & $(3.00,45.7,45$. \\
\hline & & $(3$. & $(3.11,126.7,2$ & $(3.15,107.9,147.6)$ & $(3.14,100.6,113.8)$ & $(3.03,91.4,100.0)$ & $(2.91,65.6,82.3$ & $(2.91,34.6,34$ \\
\hline & 1.5 & $(3.00,37.9,91.7)$ & $(3.00,30.0,47.6)$ & $(3.00,27.3,34.1)$ & $(3.00,25.9,31.9)$ & $(3.00,24.1,29.8)$ & $(3.00,18.6,21.8)$ & $(3.00,15.8,15.2)$ \\
\hline & & $(3.13,48.5,124.8)$ & $(3.11,37.2,58.8)$ & $(3.15,33.6,41.2)$ & $(3.14,32.1,34.4)$ & $(3.03,29.6,31.4)$ & $(2.91,22.3,26.0)$ & $(2.91,13.6,13.0)$ \\
\hline & 2.0 & $(3.00,7.4,10.9)$ & $(3.00,6.6,7.8)$ & $(3.00,6.4,6.7)$ & $(3.00,6.2,6.4)$ & $(3.00,5.9,6.0)$ & $(3.00,5.0,4.8)$ & $(3.00,4.6,4.0)$ \\
\hline & & $(3.13,9.6,15.6)$ & $(3.11,8.4,10.2)$ & $(3.15,8.0,8.4)$ & $(3.14,7.9,7.7)$ & $(3.03,7.4,7.2)$ & $(2.91,6.0,6.1)$ & $(2.91,4.4,3.8)$ \\
\hline \multirow[t]{8}{*}{0.10} & 1.1 & $00,270.2,664.4)$ & $00,202.0,379$ & $.00,165.4,222$ & $(3.00,159.6$, & $3.00,163.4,19$ & $(3.00,178.2,20$ & $(3.00,228.1,22$ \\
\hline & & $05,298.6,936.4)$ & $8,224.1,420.1)$ & $(3.08,178.5,242.9)$ & $(3.11,172.3,213.9)$ & $(3.16,173.0,209.6)$ & $(3.25,189.0,236.8)$ & $(3.00,144.1,143.5)$ \\
\hline & 1.2 & $.00,118.0,258.0)$ & $(3.00,92.2,156.3)$ & $(3.00,78.4,100.1)$ & $(3.00,76.2,89.7)$ & $(3.00,77.9,90.3)$ & $(3.00,84.4,94.8)$ & $(3.00,106.3,105.7)$ \\
\hline & & $(3.05,135.8,368.0)$ & $(3.08,107.6,184.2)$ & $(3.08,88.8,115.0)$ & $(3.11,86.4,103.0)$ & $(3.16,86.8,101.3)$ & $(3.25,94.0,113.1)$ & $(3.00,74.0,73.4)$ \\
\hline & 1.5 & $(3.00,18.8,32.8)$ & $(3.00,16.2,21.5)$ & $(3.00,14.8,16.5)$ & $(3.00,14.6,15.6)$ & $(3.00,14.8,15.8)$ & $(3.00,15.8,16.5)$ & $(3.00,18.9,18.4)$ \\
\hline & & $(3.05,22.7,43.0)$ & $(3.08,20.1,27.5)$ & $(3.08,17.9,20.4)$ & $(3.11,17.7,19.0)$ & $(3.16,17.8,18.8)$ & $(3.25,18.8,20.4)$ & $(3.00,16.0,15.4)$ \\
\hline & 2.0 & $(3.00,3.4,3.6)$ & $(3.00,3.2,3.0)$ & $(3.00,3.1,2.7)$ & $(3.00,3.1,2.6)$ & $(3.00,3.1,2.6)$ & $(3.00,3.2,2.7)$ & $(3.00,3.6,3.0)$ \\
\hline & & $(3.05,4.0,4.7)$ & $(3.08,3.9,3.9)$ & $(3.08,3.7,3.3)$ & $(3.11,3.7,3.2)$ & $(3.16,3.7,3.2)$ & $(3.25,3.8,3.4)$ & $(3.00,3.5,2.9)$ \\
\hline \multirow[t]{8}{*}{0.20} & 1.1 & $(3.00,181.1,275.9)$ & $.00,165.2,238.4)$ & $(3.00,149.3,184.8)$ & $(3.00,145.2,166.8)$ & $(3.00,147.3,166.4)$ & $(3.00,161.9,177.9)$ & $(3.00,198.7,198.1)$ \\
\hline & & $(2.99,181.5,299.7)$ & $(2.99,159.2,231.9)$ & $(2.99,144.2,178.5)$ & $(3.01,142.1,163.6)$ & $(3.05,145.4,161.0)$ & $(3.15,172.6,202.7)$ & $(3.00,131.7,131.1)$ \\
\hline & 1.2 & $(3.00,74.7,133.0)$ & $(3.00,62.5,91.2)$ & $(3.00,55.7,66.7)$ & $(3.00,54.2,60.6)$ & $(3.00,54.8,60.3)$ & $(3.00,59.1,63.5)$ & $(3.00,70.6,70.0)$ \\
\hline & & $(2.99,78.0,142.6)$ & $(2.99,63.7,93.9)$ & $(2.99,56.8,68.1)$ & $(3.01,55.8,62.0)$ & $(3.05,57.0,61.2)$ & $(3.15,66.1,74.8)$ & $(3.00,52.4,51.8)$ \\
\hline & 1.5 & $(3.00,7.7,10.5)$ & $(3.00,7.0,7.9)$ & $(3.00,6.7,6.7)$ & $(3.00,6.6,6.4)$ & $(3.00,6.6,6.4)$ & $(3.00,7.0,6.7)$ & $(3.00,7.8,7.3)$ \\
\hline & & $(2.99,8.8,12.7)$ & $(2.99,7.8,9.0)$ & $(2.99,7.4,7.5)$ & $(3.01,7.4,7.2)$ & $(3.05,7.5,7.2)$ & $(3.15,8.2,8.1)$ & $(3.00,7.2,6.6)$ \\
\hline & 2.0 & $(3.00,1.5,0.9)$ & $(3.00,1.5,0.8)$ & $(3.00,1.5,0.8)$ & $(3.00,1.5,0.8)$ & $(3.00,1.5,0.8)$ & $(3.00,1.5,0.8)$ & $(3.00,1.5,0.8)$ \\
\hline & & $(2.99,1.6,1.1)$ & $(2.99,1.6,1.0)$ & $(2.99,1.6,0.9)$ & $(3.01,1.6,0.9)$ & $(3.05,1.6,0.9)$ & $(3.15,1.6,1.0)$ & $(3.00,1.6,0.9)$ \\
\hline
\end{tabular}


Table 22: $\left(K, \mathrm{ARL}_{1}, \mathrm{SDRL}_{1}\right)$ for the hypergeometric $n p$ chart and $\left(K^{\prime \prime}, \mathrm{ARL}_{1}, \mathrm{SDRL}_{1}\right)$ for the binomial $n p$ chart with $N=1000, n \in\{25,50\}, p_{0} \in\{0.01,0.05,0.10,0.20\}, m \in\{10,20,50,100,200,1000, \infty\}$

\begin{tabular}{|c|c|c|c|c|c|c|c|c|}
\hline$p_{0}$ & $\tau$ & $m=10$ & $m=20$ & $m=50$ & $m=100$ & $m=200$ & $m=1000$ & $m \rightarrow \infty$ \\
\hline \multicolumn{9}{|c|}{$n=25$} \\
\hline 0.01 & 1.2 & $\begin{array}{c}(3.00,292.2,5510.3) \\
(3.13,311.6,3615.9) \\
(3.00,210.6,3061.0) \\
(3.13,235.0,2365.4) \\
(3.00,96.2,809.8) \\
(3.13,117.0,822.0) \\
(3.00,38.9,192.8) \\
(3.13,50.3,225.7)\end{array}$ & $\begin{array}{c}(3.00,160.5,712.8) \\
(3.13,181.0,506.7) \\
(3.00,123.0,494.0) \\
(3.13,142.3,373.9) \\
(3.00,64.2,203.4) \\
(3.13,77.7,176.0) \\
(3.00,29.6,71.9) \\
(3.13,36.8,70.5)\end{array}$ & $\begin{array}{c}(3.00,93.7,238.4) \\
(3.00,101.8,225.0) \\
(3.00,74.0,181.5) \\
(3.00,81.5,175.8) \\
(3.00,41.5,91.9) \\
(3.00,46.8,94.0) \\
(3.00,20.7,39.7) \\
(3.00,23.7,42.7)\end{array}$ & $\begin{array}{c}(3.00,66.4,178.3) \\
(3.00,68.1,166.0) \\
(3.00,53.4,136.0) \\
(3.00,55.4,129.9) \\
(3.00,31.3,69.3) \\
(3.00,33.1,69.8) \\
(3.00,16.5,30.4) \\
(3.00,17.7,32.1)\end{array}$ & $\begin{array}{c}(3.00,44.9,105.5) \\
(3.00,43.8,98.2) \\
(3.00,37.1,81.1) \\
(3.00,36.5,77.4) \\
(3.00,23.2,42.4) \\
(3.00,23.2,42.5) \\
(3.00,13.2,19.5) \\
(3.00,13.3,20.3)\end{array}$ & $\begin{array}{l}(3.00,34.8,34.3) \\
(3.19,34.7,51.7) \\
(3.00,29.5,28.9) \\
(3.19,29.5,41.8) \\
(3.00,19.4,18.9) \\
(3.19,19.5,24.7) \\
(3.00,11.6,11.0) \\
(3.19,11.7,13.1)\end{array}$ & $\begin{array}{l}(3.00,34.8,34.3) \\
(3.00,32.6,32.1) \\
(3.00,29.5,28.9) \\
(3.00,27.8,27.3) \\
(3.00,19.4,18.9) \\
(3.00,18.7,18.1) \\
(3.00,11.6,11.0) \\
(3.00,11.3,10.8)\end{array}$ \\
\hline 0.05 & 1.1 & $\begin{array}{c}(3.00,321.5,1502.6) \\
(3.10,345.1,1861.0) \\
(3.00,198.5,807.9) \\
(3.10,215.0,999.2) \\
(3.00,62.2,183.9) \\
(3.10,68.2,224.1) \\
(3.00,16.7,34.2) \\
(3.10,18.3,40.4)\end{array}$ & $\begin{array}{c}(3.00,186.1,445.0) \\
(3.04,189.7,442.4) \\
(3.00,121.5,268.3) \\
(3.04,125.3,272.3) \\
(3.00,43.4,79.0) \\
(3.04,45.6,83.2) \\
(3.00,13.3,19.2) \\
(3.04,14.1,20.7)\end{array}$ & $\begin{array}{c}(3.00,127.3,215.8) \\
(3.02,131.2,220.9) \\
(3.00,86.2,138.0) \\
(3.02,89.5,143.6) \\
(3.00,33.5,46.6) \\
(3.02,35.2,49.8) \\
(3.00,11.2,13.2) \\
(3.02,11.9,14.3)\end{array}$ & $\begin{array}{c}(3.00,109.0,136.3) \\
(3.05,109.7,157.5) \\
(3.00,75.2,90.5) \\
(3.05,76.1,104.2) \\
(3.00,30.4,33.7) \\
(3.05,31.1,38.1) \\
(3.00,10.6,10.7) \\
(3.05,11.0,11.8)\end{array}$ & $\begin{array}{c}(3.00,104.9,106.7) \\
(3.13,104.8,138.9) \\
(3.00,72.8,73.6) \\
(3.13,73.1,92.8) \\
(3.00,29.8,29.6) \\
(3.13,30.2,34.8) \\
(3.00,10.5,10.0) \\
(3.13,10.8,11.1)\end{array}$ & $\begin{array}{c}(3.00,104.6,104.1) \\
(3.30,106.5,144.5) \\
(3.00,72.6,72.1) \\
(3.30,74.1,96.3) \\
(3.00,29.8,29.2) \\
(3.30,30.6,35.8) \\
(3.00,10.5,10.0) \\
(3.30,10.8,11.3)\end{array}$ & $\begin{array}{c}(3.00,104.6,104.1) \\
(3.00,94.4,93.8) \\
(3.00,72.6,72.1) \\
(3.00,66.5,65.9) \\
(3.00,29.8,29.2) \\
(3.00,28.1,27.6) \\
(3.00,10.5,10.0) \\
(3.00,10.3,9.7)\end{array}$ \\
\hline 0.10 & 1.1 & $\begin{array}{c}(3.00,283.3,1260.7) \\
(2.99,295.8,1164.6) \\
(3.00,154.1,573.5) \\
(2.99,162.3,557.0) \\
(3.00,36.8,90.1) \\
(2.99,39.1,95.7) \\
(3.00,7.9,12.2) \\
(2.99,8.3,13.4)\end{array}$ & $\begin{array}{c}(3.00,197.2,407.6) \\
(3.01,196.0,398.4) \\
(3.00,113.1,214.4) \\
(3.01,113.9,213.5) \\
(3.00,30.2,46.5) \\
(3.01,31.1,47.9) \\
(3.00,7.2,8.6) \\
(3.01,7.5,9.0)\end{array}$ & $\begin{array}{c}(3.00,162.0,230.6) \\
(3.03,167.2,230.8) \\
(3.00,95.3,130.6) \\
(3.03,99.5,132.4) \\
(3.00,27.0,33.4) \\
(3.03,28.6,34.7) \\
(3.00,6.8,7.1) \\
(3.03,7.2,7.5)\end{array}$ & $\begin{array}{c}(3.00,152.8,209.2) \\
(3.01,155.2,200.2) \\
(3.00,90.3,120.0) \\
(3.01,92.9,117.1) \\
(3.00,25.8,31.5) \\
(3.01,27.1,31.9) \\
(3.00,6.6,6.9) \\
(3.01,7.0,7.2)\end{array}$ & $\begin{array}{c}(3.00,147.1,203.6) \\
(2.99,147.9,193.9) \\
(3.00,87.2,116.9) \\
(2.99,88.8,113.5) \\
(3.00,25.1,30.7) \\
(2.99,26.1,31.0) \\
(3.00,6.5,6.7) \\
(2.99,6.8,7.0)\end{array}$ & $\begin{array}{c}(3.00,122.5,176.4) \\
(2.98,128.9,176.8) \\
(3.00,73.5,101.7) \\
(2.98,78.0,103.6) \\
(3.00,21.9,27.1) \\
(2.98,23.5,28.5) \\
(3.00,5.9,6.1) \\
(2.98,6.3,6.5)\end{array}$ & $\begin{array}{c}(3.00,69.5,69.0) \\
(2.99,64.1,63.5) \\
(3.00,44.1,43.5) \\
(2.99,41.3,40.7) \\
(3.00,15.0,14.4) \\
(2.99,14.4,13.9) \\
(3.00,4.6,4.1) \\
(2.99,4.6,4.0)\end{array}$ \\
\hline 0.20 & 1.2 & $\begin{array}{c}(3.00,299.3,722.6) \\
(3.01,312.7,734.3) \\
(3.00,136.5,297.7) \\
(3.01,142.7,304.0) \\
(3.00,21.7,41.6) \\
(3.01,22.8,42.5) \\
(3.00,3.4,3.8) \\
(3.01,3.6,4.0)\end{array}$ & $\begin{array}{c}(3.00,219.5,406.3) \\
(3.00,224.5,411.9) \\
(3.00,102.0,173.4) \\
(3.00,105.5,177.3) \\
(3.00,17.4,24.0) \\
(3.00,18.4,24.8) \\
(3.00,3.1,2.9) \\
(3.00,3.3,3.1)\end{array}$ & $\begin{array}{c}(3.00,189.2,259.7) \\
(3.02,195.7,264.3) \\
(3.00,90.1,118.0) \\
(3.02,94.2,121.5) \\
(3.00,16.3,18.7) \\
(3.02,17.3,19.7) \\
(3.00,3.0,2.6) \\
(3.02,3.2,2.8)\end{array}$ & $\begin{array}{c}(3.00,178.0,232.9) \\
(3.00,183.0,227.7) \\
(3.00,85.4,107.6) \\
(3.00,88.8,107.4) \\
(3.00,15.7,17.7) \\
(3.00,16.6,18.3) \\
(3.00,3.0,2.6) \\
(3.00,3.1,2.7)\end{array}$ & $\begin{array}{c}(3.00,170.1,224.5) \\
(2.99,174.3,219.7) \\
(3.00,81.9,103.9) \\
(2.99,84.9,103.8) \\
(3.00,15.2,17.1) \\
(2.99,16.0,17.7) \\
(3.00,2.9,2.5) \\
(2.99,3.1,2.6)\end{array}$ & $\begin{array}{c}(3.00,138.9,186.7) \\
(2.97,134.6,178.4) \\
(3.00,68.2,87.2) \\
(2.97,67.0,85.0) \\
(3.00,13.3,14.8) \\
(2.97,13.4,15.0) \\
(3.00,2.8,2.3) \\
(2.97,2.8,2.3)\end{array}$ & $\begin{array}{c}(3.00,93.4,92.8) \\
(2.99,85.9,85.3) \\
(3.00,48.2,47.6) \\
(2.99,45.1,44.6) \\
(3.00,10.6,10.0) \\
(2.99,10.3,9.7) \\
(3.00,2.5,1.9) \\
(2.99,2.5,1.8)\end{array}$ \\
\hline & & & & & & & & \\
\hline 0.01 & 1.1 & $\begin{array}{c}(3.00,339.5,5033.5) \\
(3.25,358.4,3397.2) \\
(3.00,223.4,2472.7) \\
(3.25,251.7,2003.8) \\
(3.00,84.2,516.5) \\
(3.25,105.6,553.4) \\
(3.00,28.3,98.2) \\
(3.25,37.6,123.1) \\
\end{array}$ & $\begin{array}{c}(3.00,154.6,603.3) \\
(3.11,143.9,451.3) \\
(3.00,110.5,381.4) \\
(3.11,107.1,310.8) \\
(3.00,49.7,129.7) \\
(3.11,51.6,123.8) \\
(3.00,19.8,38.4) \\
(3.11,21.5,41.1) \\
\end{array}$ & $\begin{array}{c}(3.00,108.4,249.7) \\
(3.09,107.9,217.9) \\
(3.00,80.3,173.4) \\
(3.09,82.1,159.5) \\
(3.00,39.0,71.4) \\
(3.09,41.8,72.9) \\
(3.00,16.8,25.1) \\
(3.09,18.5,27.9)\end{array}$ & $\begin{array}{c}(3.00,77.5,133.0) \\
(3.06,77.5,146.6) \\
(3.00,59.4,94.6) \\
(3.06,60.2,108.1) \\
(3.00,31.0,42.0) \\
(3.06,32.2,50.7) \\
(3.00,14.4,16.6) \\
(3.06,15.2,20.3)\end{array}$ & $\begin{array}{c}(3.00,69.2,75.7) \\
(3.15,69.6,121.2) \\
(3.00,53.7,57.5) \\
(3.15,54.5,90.0) \\
(3.00,28.8,29.5) \\
(3.15,29.7,43.0) \\
(3.00,13.7,13.4) \\
(3.15,14.3,17.7)\end{array}$ & $\begin{array}{c}(3.00,68.5,67.9) \\
(3.36,68.9,118.6) \\
(3.00,53.2,52.7) \\
(3.36,54.0,88.1) \\
(3.00,28.6,28.1) \\
(3.36,29.5,42.2) \\
(3.00,13.6,13.1) \\
(3.36,14.2,17.5)\end{array}$ & $\begin{array}{l}(3.00,68.5,67.9) \\
(3.00,56.3,55.8) \\
(3.00,53.2,52.7) \\
(3.00,44.9,44.4) \\
(3.00,28.6,28.1) \\
(3.00,25.5,25.0) \\
(3.00,13.6,13.1) \\
(3.00,12.8,12.2)\end{array}$ \\
\hline 0.05 & 1.1 & $\begin{array}{c}(3.00,286.3,1194.6) \\
(3.05,300.5,1201.0) \\
(3.00,154.0,531.0) \\
(3.05,165.7,570.8) \\
(3.00,36.5,82.9) \\
(3.05,40.7,98.1) \\
(3.00,8.1,11.9) \\
(3.05,9.0,14.2)\end{array}$ & $\begin{array}{c}(3.00,195.0,406.4) \\
(3.09,208.1,426.1) \\
(3.00,110.7,211.0) \\
(3.09,120.8,228.6) \\
(3.00,29.5,45.4) \\
(3.09,33.2,51.8) \\
(3.00,7.2,8.6) \\
(3.09,8.1,10.0)\end{array}$ & $\begin{array}{c}(3.00,150.8,210.3) \\
(3.07,158.5,221.8) \\
(3.00,88.7,118.6) \\
(3.07,95.2,127.5) \\
(3.00,25.4,30.7) \\
(3.07,28.3,34.0) \\
(3.00,6.7,6.9) \\
(3.07,7.5,7.7)\end{array}$ & $\begin{array}{c}(3.00,146.2,187.6) \\
(3.06,150.2,172.6) \\
(3.00,86.3,108.0) \\
(3.06,91.1,102.9) \\
(3.00,25.0,29.0) \\
(3.06,27.6,29.7) \\
(3.00,6.6,6.7) \\
(3.06,7.4,7.2)\end{array}$ & $\begin{array}{c}(3.00,145.6,186.3) \\
(3.03,150.6,166.4) \\
(3.00,86.0,107.4) \\
(3.03,91.4,100.0) \\
(3.00,24.9,28.9) \\
(3.03,27.7,29.3) \\
(3.00,6.6,6.7) \\
(3.03,7.4,7.2)\end{array}$ & $\begin{array}{c}(3.00,150.5,190.0) \\
(2.98,157.7,169.5) \\
(3.00,88.7,109.5) \\
(2.98,95.5,101.9) \\
(3.00,25.6,29.5) \\
(2.98,28.8,29.8) \\
(3.00,6.7,6.8) \\
(2.98,7.6,7.3)\end{array}$ & $\begin{array}{c}(3.00,227.2,226.6) \\
(3.00,176.9,176.4) \\
(3.00,131.4,130.8) \\
(3.00,106.7,106.1) \\
(3.00,35.8,35.2) \\
(3.00,31.7,31.1) \\
(3.00,8.6,8.1) \\
(3.00,8.2,7.7)\end{array}$ \\
\hline 0.10 & 1.1 & $\begin{array}{c}(3.00,274.3,812.1) \\
(3.04,279.8,900.5) \\
(3.00,122.2,313.7) \\
(3.04,128.1,353.0) \\
(3.00,20.0,36.9) \\
(3.04,21.8,41.3) \\
(3.00,3.6,4.0) \\
(3.04,3.9,4.5)\end{array}$ & $\begin{array}{c}(3.00,189.1,356.2) \\
(3.04,192.9,358.6) \\
(3.00,89.7,152.7) \\
(3.04,94.0,158.9) \\
(3.00,16.7,22.3) \\
(3.04,18.2,24.5) \\
(3.00,3.4,3.2) \\
(3.04,3.7,3.6)\end{array}$ & $\begin{array}{c}(3.00,156.3,210.4) \\
(3.04,163.0,219.1) \\
(3.00,76.7,97.9) \\
(3.04,81.8,104.7) \\
(3.00,15.3,17.1) \\
(3.04,16.8,19.0) \\
(3.00,3.3,2.9) \\
(3.04,3.6,3.2)\end{array}$ & $\begin{array}{c}(3.00,151.5,173.4) \\
(3.06,154.9,185.3) \\
(3.00,75.1,83.9) \\
(3.06,78.5,90.6) \\
(3.00,15.3,15.8) \\
(3.06,16.5,17.3) \\
(3.00,3.3,2.8) \\
(3.06,3.6,3.1)\end{array}$ & $\begin{array}{c}(3.00,157.6,169.3) \\
(3.13,161.6,187.5) \\
(3.00,78.0,82.8) \\
(3.13,81.8,91.8) \\
(3.00,15.8,15.9) \\
(3.13,17.1,17.6) \\
(3.00,3.4,2.8) \\
(3.13,3.6,3.1)\end{array}$ & $\begin{array}{c}(3.00,176.0,177.0) \\
(3.24,177.2,217.1) \\
(3.00,86.5,86.6) \\
(3.24,88.7,104.5) \\
(3.00,17.1,16.7) \\
(3.24,18.1,19.3) \\
(3.00,3.5,2.9) \\
(3.24,3.7,3.3)\end{array}$ & $\begin{array}{c}(3.00,178.5,177.9) \\
(3.00,144.1,143.5) \\
(3.00,87.6,87.1) \\
(3.00,74.0,73.4) \\
(3.00,17.3,16.7) \\
(3.00,16.0,15.4) \\
(3.00,3.6,3.0) \\
(3.00,3.5,2.9)\end{array}$ \\
\hline 0.20 & 1.1 & $\begin{array}{c}(3.00,187.6,303.5) \\
(2.99,181.5,299.7) \\
(3.00,78.0,139.0) \\
(2.99,78.0,142.6) \\
(3.00,8.4,11.6) \\
(2.99,8.8,12.7) \\
(3.00,1.6,1.0) \\
(2.99,1.6,1.1)\end{array}$ & $\begin{array}{c}(3.00,165.1,241.2) \\
(2.99,159.2,231.9) \\
(3.00,64.3,94.5) \\
(2.99,63.7,93.9) \\
(3.00,7.5,8.5) \\
(2.99,7.8,9.0) \\
(3.00,1.5,0.9) \\
(2.99,1.6,1.0)\end{array}$ & $\begin{array}{c}(3.00,147.8,181.2) \\
(2.99,144.2,178.5) \\
(3.00,56.9,68.3) \\
(2.99,56.8,68.1) \\
(3.00,7.1,7.1) \\
(2.99,7.4,7.5) \\
(3.00,1.5,0.8) \\
(2.99,1.6,0.9)\end{array}$ & $\begin{array}{c}(3.00,143.0,158.9) \\
(3.02,146.4,170.1) \\
(3.00,54.8,59.8) \\
(3.02,57.2,64.0) \\
(3.00,7.0,6.7) \\
(3.02,7.5,7.3) \\
(3.00,1.5,0.8) \\
(3.02,1.6,0.9)\end{array}$ & $\begin{array}{c}(3.00,147.0,153.9) \\
(3.07,152.7,173.4) \\
(3.00,56.0,57.8) \\
(3.07,59.4,65.2) \\
(3.00,7.1,6.7) \\
(3.07,7.7,7.5) \\
(3.00,1.5,0.8) \\
(3.07,1.6,0.9)\end{array}$ & $\begin{array}{c}(3.00,159.0,158.9) \\
(3.15,172.6,202.7) \\
(3.00,60.0,59.6) \\
(3.15,66.1,74.8) \\
(3.00,7.5,6.9) \\
(3.15,8.2,8.1) \\
(3.00,1.5,0.9) \\
(3.15,1.6,1.0)\end{array}$ & $\begin{array}{c}(3.00,159.8,159.2) \\
(3.00,131.7,131.1) \\
(3.00,60.3,59.7) \\
(3.00,52.4,51.8) \\
(3.00,7.5,6.9) \\
(3.00,7.2,6.6) \\
(3.00,1.5,0.9) \\
(3.00,1.6,0.9)\end{array}$ \\
\hline
\end{tabular}


Table 23: $\left(K, \mathrm{ARL}_{1}, \mathrm{SDRL}_{1}\right)$ for the hypergeometric $n p$ chart and $\left(K^{\prime \prime}, \mathrm{ARL}_{1}, \mathrm{SDRL}_{1}\right)$ for the binomial $n p$ chart with $N=2000, n \in\{25,50\}, p_{0} \in\{0.01,0.05,0.10,0.20\}, m \in\{10,20,50,100,200,1000, \infty\}$

\begin{tabular}{|c|c|c|c|c|c|c|c|c|}
\hline$p_{0}$ & $\tau$ & $m=10$ & $m=20$ & $m=50$ & $m=100$ & $m=200$ & $m=1000$ & $m \rightarrow \infty$ \\
\hline \multicolumn{9}{|c|}{$n=25$} \\
\hline \multirow[t]{8}{*}{0.01} & 1.1 & $(3.00,243.0,3237.6)$ & $(3.00,142.4,568.0)$ & $(3.00,85.6,210.4)$ & $(3.00,61.8,158.2)$ & $(3.00,42.7,94.8)$ & $(3.00,33.7,33.1)$ & $(3.00,33.7,33.1)$ \\
\hline & & $(3.10,234.5,3611.3)$ & $(3.10,128.2,477.9)$ & $(2.98,79.0,188.0)$ & $(2.98,57.9,142.0)$ & $(3.00,43.8,98.2)$ & $(3.16,33.7,42.8)$ & $(3.00,32.6,32.1)$ \\
\hline & 1.2 & $(3.00,180.3,1996.8)$ & $(3.00,111.0,406.9)$ & $(3.00,68.5,162.5)$ & $(3.00,50.2,122.4)$ & $(3.00,35.6,73.9)$ & $(3.00,28.6,28.1)$ & $(3.00,28.6,28.1)$ \\
\hline & & $(3.10,175.1,2361.6)$ & $(3.10,101.3,349.9)$ & $(2.98,63.8,147.0)$ & $(2.98,47.5,111.3)$ & $(3.00,36.5,77.4)$ & $(3.16,28.6,35.1)$ & $(3.00,27.8,27.3)$ \\
\hline & 1.5 & $(3.00,87.1,633.4)$ & $(3.00,59.9,179.0)$ & $(3.00,39.4,84.8)$ & $(3.00,30.0,64.3)$ & $(3.00,22.6,39.9)$ & $(3.00,19.0,18.5)$ & $(3.00,19.0,18.5)$ \\
\hline & & $(3.10,85.6,818.8)$ & $(3.10,56.2,161.2)$ & $(2.98,37.5,78.8)$ & $(2.98,29.0,60.1)$ & $(3.00,23.2,42.5)$ & $(3.16,19.1,21.6)$ & $(3.00,18.7,18.1)$ \\
\hline & 2.0 & $(3.00,36.8,170.5)$ & $(3.00,28.4,66.9)$ & $(3.00,20.2,37.8)$ & $(3.00,16.2,29.0)$ & $(3.00,13.0,18.9)$ & $(3.00,11.5,10.9)$ & $(3.00,11.5,10.9)$ \\
\hline & & $(3.10,36.5,223.1)$ & $(3.10,27.4,62.8)$ & $(2.98,19.6,36.0)$ & $(2.98,15.9,27.8)$ & $(3.00,13.3,20.3)$ & $(3.16,11.5,12.0)$ & $(3.00,11.3,10.8)$ \\
\hline \multirow[t]{8}{*}{0.05} & 1.1 & $(3.00,293.9,1439.7)$ & $(3.00,173.1,403.4)$ & $(3.00,121.6,199.7)$ & $(3.00,105.3,134.3)$ & $(3.00,99.8,102.5)$ & $(3.00,99.3,98.7)$ & $(3.00,99.3,98.7)$ \\
\hline & & $(3.08,288.6,1752.0)$ & $3.03,166.9,420.1)$ & $(3.01,121.7,202.7)$ & $(3.02,106.7,148.4)$ & $(3.09,100.0,120.5)$ & $(3.27,98.6,114.2)$ & $(3.00,94.4,93.8)$ \\
\hline & 1.2 & $(3.00,184.0,775.3)$ & $(3.00,114.3,247.0)$ & $(3.00,83.1,129.3)$ & $(3.00,73.1,89.7)$ & $(3.00,69.8,71.0)$ & $(3.00,69.4,68.9)$ & $(3.00,69.4,68.9)$ \\
\hline & & $(3.08,182.2,928.8)$ & $(3.03,111.0,257.4)$ & $(3.01,83.5,132.2)$ & $(3.02,74.2,98.6)$ & $(3.09,70.1,81.7)$ & $(3.27,69.2,78.0)$ & $(3.00,66.5,65.9)$ \\
\hline & 1.5 & $(3.00,59.4,177.4)$ & $(3.00,41.8,75.0)$ & $(3.00,32.8,44.8)$ & $(3.00,30.0,33.7)$ & $(3.00,29.0,28.9)$ & $(3.00,28.9,28.4)$ & $(3.00,28.9,28.4)$ \\
\hline & & $(3.08,59.9,201.9)$ & $(3.03,41.1,77.9)$ & $(3.01,33.2,46.3)$ & $(3.02,30.5,36.5)$ & $(3.09,29.3,31.8)$ & $(3.27,29.0,30.7)$ & $(3.00,28.1,27.6)$ \\
\hline & 2.0 & $(3.00,16.3,33.4)$ & $(3.00,13.1,18.8)$ & $(3.00,11.2,13.0)$ & $(3.00,10.6,10.8)$ & $(3.00,10.4,9.9)$ & $(3.00,10.4,9.8)$ & $(3.00,10.4,9.8)$ \\
\hline & & $(3.08,16.7,35.7)$ & $(3.03,13.0,19.3)$ & $(3.01,11.4,13.5)$ & $(3.02,10.8,11.4)$ & $(3.09,10.5,10.5)$ & $(3.27,10.5,10.3)$ & $(3.00,10.3,9.7)$ \\
\hline \multirow[t]{8}{*}{0.10} & 1.1 & $(3.00,297.2,1165.8)$ & $(3.00,196.3,412.4)$ & $(3.00,160.8,225.5)$ & $(3.00,154.3,204.9)$ & $(3.00,149.5,200.5)$ & $(3.00,137.7,189.6)$ & $(3.00,66.7,66.1)$ \\
\hline & & $2.99,295.8,1$ & $(3.01,196.0,3$ & $(3.02,160.5,226.7)$ & $(3.01,155.2,200.2)$ & $(2.99,147.9,19:$ & $(2.99,140.5,187.7)$ & $(2.99,64.1,63.5)$ \\
\hline & 1.2 & $(3.00,162.5,545.7)$ & $(3.00,113.1,219.1)$ & $(3.00,95.3,128.8)$ & $(3.00,91.8,118.7)$ & $(3.00,89.1,116.3)$ & $(3.00,82.5,110.0)$ & $(3.00,42.6,42.1)$ \\
\hline & & $(2.99,162.3,557.0)$ & $(3.01,113.9,213.5)$ & $(3.02,95.7,129.9)$ & $(3.01,92.9,117.1)$ & $(2.99,88.8,113.5)$ & $(2.99,84.6,109.9)$ & $(2.99,41.3,40.7)$ \\
\hline & 1.5 & $(3.00,38.9,91.0)$ & $(3.00,30.4,48.2)$ & $(3.00,27.3,33.5)$ & $(3.00,26.5,31.7)$ & $(3.00,25.9,31.1)$ & $(3.00,24.3,29.6)$ & $(3.00,14.7,14.1)$ \\
\hline & & $(2.99,39.1,95.7)$ & $(3.01,31.1,47.9)$ & $(3.02,27.7,34.0)$ & $(3.01,27.1,31.9)$ & $(2.99,26.1,31.0)$ & $(2.99,25.1,30.1)$ & $(2.99,14.4,13.9)$ \\
\hline & 2.0 & $(3.00,8.3,12.8)$ & $(3.00,7.3,8.9)$ & $(3.00,6.9,7.3)$ & $(3.00,6.8,7.0)$ & $(3.00,6.7,6.9)$ & $(3.00,6.4,6.6)$ & $(3.00,4.6,4.0)$ \\
\hline & & $(2.99,8.3,13.4)$ & $(3.01,7.5,9.0)$ & $(3.02,7.0,7.4)$ & $(3.01,7.0,7.2)$ & $(2.99,6.8,7.0)$ & $(2.99,6.6,6.8)$ & $(2.99,4.6,4.0)$ \\
\hline \multirow[t]{8}{*}{0.20} & 1.1 & $(3.00,280.8,666.6)$ & $(3.00,226.9,417.7)$ & $(3.00,185.8,253.1)$ & $(3.00,180.8,230.8)$ & $(3.00,178.1,227.8)$ & $(3.00,160.3,210.6)$ & $(3.00,89.5,88.9)$ \\
\hline & & $(3.00,287.7,699.2)$ & $(3.00,224.5,411.9)$ & $(3.00,188.0,250.8)$ & $(3.00,183.0,227.7)$ & $(3.00,182.3,226.4)$ & $(2.99,165.0,211.5)$ & $(2.99,85.9,85.3)$ \\
\hline & 1.2 & $(3.00,129.9,279.6)$ & $(3.00,105.7,179.0)$ & $(3.00,89.2,116.1)$ & $(3.00,87.2,107.7)$ & $(3.00,86.0,106.5)$ & $(3.00,78.1,98.7)$ & $(3.00,46.6,46.0)$ \\
\hline & & $(3.00,132.4,288.9)$ & $(3.00,105.5,177.3)$ & $(3.00,90.8,116.1)$ & $(3.00,88.8,107.4)$ & $(3.00,88.5,106.8)$ & $(2.99,80.7,100.0)$ & $(2.99,45.1,44.6)$ \\
\hline & 1.5 & $(3.00,21.3,40.3)$ & $(3.00,18.1,24.8)$ & $(3.00,16.4,18.7)$ & $(3.00,16.1,18.0)$ & $(3.00,16.0,17.8)$ & $(3.00,14.9,16.7)$ & $(3.00,10.4,9.9)$ \\
\hline & & $(3.00,21.6,39.5)$ & $(3.00,18.4,24.8)$ & $(3.00,16.8,19.1)$ & $(3.00,16.6,18.3)$ & $(3.00,16.5,18.2)$ & $(2.99,15.4,17.2)$ & $(2.99,10.3,9.7)$ \\
\hline & 2.0 & $(3.00,3.4,3.8)$ & $(3.00,3.2,3.0)$ & $(3.00,3.1,2.7)$ & $(3.00,3.1,2.6)$ & $(3.00,3.0,2.6)$ & $(3.00,2.9,2.5)$ & $(3.00,2.5,1.9)$ \\
\hline & & $(3.00,3.5,3.8)$ & $(3.00,3.3,3.1)$ & $(3.00,3.2,2.8)$ & $(3.00,3.1,2.7)$ & $(3.00,3.1,2.7)$ & $(2.99,3.0,2.6)$ & $(2.99,2.5,1.8)$ \\
\hline \multicolumn{9}{|c|}{$n=50$} \\
\hline \multirow[t]{8}{*}{0.01} & 1.1 & $(3.00,251.8,2402.5)$ & $(3.00,163.3,463.8)$ & $(3.00,93.2,198.5)$ & $(3.00,72.1,123.6)$ & $(3.00,63.0,71.7)$ & $(3.00,61.8,6$ & $(3.00,61.8,61.2)$ \\
\hline & & $0.9)$ & & $(3.0$ & $(3.04$ & $(3.0$ & $(3.33$ & $(3.00$ \\
\hline & 1.2 & $, 174.6,1381.5)$ & $(3.00,119.3,314.0)$ & $(3.00,70.7,142.3)$ & $(3.00,:$ & $9.6,54.9)$ & $(3.00,48.7,48.1)$ & $(3.00,48.7,48.1)$ \\
\hline & & $1,190.7,1209.1)$ & $(3.12,136.0,330.9)$ & $(3.04,71.9,139.0)$ & (3.04, & $9.9,71.7)$ & (3.33, & $(3.00,44.9,44.4)$ \\
\hline & 1.5 & $(3.00,72.5,372.3)$ & $(3.00,55.3,121.9)$ & $(3.00,36.0,62.7)$ & $0.0,41.7)$ & $(3.00,27.3,28.7)$ & $(3.00,27.0,26.4)$ & $(3.00,27.0,26.4)$ \\
\hline & & $(3.04,80.6,374.5)$ & $(3.12,64.6,134.0)$ & $.3,64.0)$ & $(3.04$, & $.7,35.5)$ & $(3.33$, & $(3.00,25.5,25.0)$ \\
\hline & 2.0 & $(3.00,26.2,84.2)$ & $(3.00,22.3,40.1)$ & $(3.00,16.1,23.4)$ & $2,16.9)$ & $(3.00,13.3,13.2)$ & $(3.00,13.2,12.6)$ & $(3.00,13.2,12.6)$ \\
\hline & & $(3.04,29.1,95.1)$ & $(3.12,26.2,45.3)$ & $(3.04,16.9,24.8)$ & $(3.04,14.6,18.7)$ & $(3.09,13.6,15.3)$ & $(3.33,13.5,15.0)$ & $(3.00,12.8,12.2)$ \\
\hline \multirow[t]{8}{*}{0.05} & 1.1 & $(3.00,270.8,1153.9)$ & $(3.00,172.8,361.6)$ & $(3.00,141.5,197.9)$ & $(3.00,141.0,173.3)$ & $(3.00,147.3,176.0)$ & $(3.00,166.6,186.5)$ & $(3.00,199.5,199.0)$ \\
\hline & & $(3.1$ & $.4)$ & $3.3)$ & $(3.04$, & 3.4) & $(3.01,1$ & $(3.00,176.9,176.4)$ \\
\hline & 1.2 & $(3.00,147.8,534.0)$ & $(3.00,100.4,191.8)$ & $(3.00,84.6,113.4)$ & $(3.00,84.6,101.9)$ & $(3.00,88.3,103.7)$ & $(3.00,99.2,109.9)$ & $(3.00,117.9,117.4)$ \\
\hline & & $(3.04,144.6,480.0)$ & $(3.02,101.1,190.3)$ & $(3.02,86.5,112.6)$ & $(3.04,87.8,100.3)$ & $(3.03,91.4,100.0)$ & $(3.01,102.0,104.5)$ & $(3.00,106.7,106.1)$ \\
\hline & 1.5 & $3.00,36.0,87.9)$ & $(3.00,28.0,43.1)$ & $(3.00,25.1,30.3)$ & $(3.00,25.2,28.6)$ & $(3.00,26.2,29.1)$ & $(3.00,28.9,30.8)$ & $(3.00,33.6,33.1)$ \\
\hline & & $.04,36.7,84.2)$ & $(3.02,28.8,44.3)$ & $(3.02,26.2,30.9)$ & $(3.04,26.7,29.0)$ & $(3.03,27.7,29.3)$ & $(3.01,30.5,30.6)$ & $(3.00,31.7,31.1)$ \\
\hline & 2.0 & $(3.00,8.1,12.6)$ & $(3.00,7.1,8.4)$ & $(3.00,6.7,7.0)$ & $(3.00,6.8,6.8)$ & $(3.00,7.0,6.9)$ & $(3.00,7.5,7.3)$ & $(3.00,8.4,7.9)$ \\
\hline & & $(3.04,8.4,12.8)$ & $(3.02,7.4,8.8)$ & $(3.02,7.1,7.3)$ & $(3.04,7.2,7.1)$ & $(3.03,7.4,7.2)$ & $(3.01,8.0,7.5)$ & $(3.00,8.2,7.7)$ \\
\hline \multirow[t]{8}{*}{0.10} & 1.1 & $(3.00,256.0,694.6)$ & $(3.00,183.0,338.5)$ & $(3.00,151.8,200.8)$ & $(3.00,145.2,165.0)$ & $(3.00,149.2,155.8)$ & $(3.00,159.5,159.1)$ & $(3.00,159.8,159.2)$ \\
\hline & & $(3.00,254.4,757.1)$ & $(3.01,185.7,336.8)$ & $(3.02,152.9,204.5)$ & $(3.03,147.4,172.1)$ & $(3.09,151.6,165.6)$ & $(3.22,160.0,183.4)$ & $(3.00,144.1,143.5)$ \\
\hline & 1.2 & $(3.00,117.1,279.3)$ & $(3.00,88.4,148.5)$ & $(3.00,75.8,95.4)$ & $(3.00,73.2,81.1)$ & $(3.00,75.3,77.9)$ & $(3.00,80.2,79.7)$ & $(3.00,80.3,79.8)$ \\
\hline & & $(3.00,117.8,303.5)$ & $(3.01,90.8,150.6)$ & $(3.02,77.2,98.3)$ & $(3.03,75.1,84.9)$ & $(3.09,77.3,82.5)$ & $(3.22,81.1,90.0)$ & $(3.00,74.0,73.4)$ \\
\hline & 1.5 & $(3.00,20.1,35.5)$ & $(3.00,17.0,22.6)$ & $(3.00,15.6,17.3)$ & $(3.00,15.4,15.8)$ & $(3.00,15.8,15.6)$ & $(3.00,16.6,16.0)$ & $(3.00,16.6,16.0)$ \\
\hline & & $(3.00,20.6,37.6)$ & $(3.01,17.7,23.7)$ & $(3.02,16.1,18.1)$ & $(3.03,16.0,16.6)$ & $(3.09,16.4,16.5)$ & $(3.22,17.0,17.4)$ & $(3.00,16.0,15.4)$ \\
\hline & 2.0 & $(3.00,3.7,4.1)$ & $(3.00,3.5,3.3)$ & $(3.00,3.4,2.9)$ & $(3.00,3.4,2.8)$ & $(3.00,3.4,2.9)$ & $(3.00,3.5,2.9)$ & $(3.00,3.5,2.9)$ \\
\hline & & $(3.00,3.8,4.3)$ & $(3.01,3.6,3.5)$ & $(3.02,3.5,3.1)$ & $(3.03,3.5,3.0)$ & $(3.09,3.6,3.0)$ & $(3.22,3.6,3.1)$ & $(3.00,3.5,2.9)$ \\
\hline \multirow[t]{8}{*}{0.20} & 1.1 & $(3.00,187.1,304.5)$ & $(3.00,162.5,234.9)$ & $(3.00,147.3,181.2)$ & $(3.00,140.6,157.2)$ & $(3.00,140.5,145.4)$ & $(3.00,144.6,144.1)$ & $(3.00,144.6,144.1)$ \\
\hline & & $(2.99,181.5,299.7)$ & $(2.98,156.6,224.2)$ & $(2.99,144.2,178.5)$ & $(3.01,142.1,163.6)$ & $(3.05,145.4,161.0)$ & $(3.13,151.3,170.6)$ & $(3.00,131.7,131.1)$ \\
\hline & 1.2 & $(3.00,78.6,140.5)$ & $(3.00,64.3,93.9)$ & $(3.00,57.4,68.8)$ & $(3.00,54.7,59.9)$ & $(3.00,54.6,55.8)$ & $(3.00,56.1,55.5)$ & $(3.00,56.1,55.5)$ \\
\hline & & $(2.99,78.0,142.6)$ & $(2.98,62.9,90.5)$ & $(2.99,56.8,68.1)$ & $(3.01,55.8,62.0)$ & $(3.05,57.0,61.2)$ & $(3.13,59.0,64.3)$ & $(3.00,52.4,51.8)$ \\
\hline & 1.5 & $(3.00,8.6,12.2)$ & $(3.00,7.7,8.8)$ & $(3.00,7.3,7.4)$ & $(3.00,7.1,6.9)$ & $(3.00,7.2,6.7)$ & $(3.00,7.3,6.7)$ & $(3.00,7.3,6.7)$ \\
\hline & & $(2.99,8.8,12.7)$ & $(2.98,7.8,8.8)$ & $(2.99,7.4,7.5)$ & $(3.01,7.4,7.2)$ & $(3.05,7.5,7.2)$ & $(3.13,7.7,7.4)$ & $(3.00,7.2,6.6)$ \\
\hline & 2.0 & $(3.00,1.6,1.0)$ & $(3.00,1.6,0.9)$ & $(3.00,1.5,0.9)$ & $(3.00,1.5,0.9)$ & $(3.00,1.5,0.9)$ & $(3.00,1.6,0.9)$ & $(3.00,1.6,0.9)$ \\
\hline & & $(2.99,1.6,1.1)$ & $(2.98,1.6,1.0)$ & $(2.99,1.6,0.9)$ & $(3.01,1.6,0.9)$ & $(3.05,1.6,0.9)$ & $(3.13,1.6,0.9)$ & $(3.00,1.6,0.9)$ \\
\hline
\end{tabular}


Table 24: $\left(K, \mathrm{ARL}_{1}, \mathrm{SDRL}_{1}\right)$ for the hypergeometric $n p$ chart and $\left(K^{\prime \prime}, \mathrm{ARL}_{1}, \mathrm{SDRL}_{1}\right)$ for the binomial $n p$ chart with $N=5000, n \in\{25,50\}, p_{0} \in\{0.01,0.05,0.10,0.20\}, m \in\{10,20,50,100,200,1000, \infty\}$

\begin{tabular}{|c|c|c|c|c|c|c|c|c|}
\hline$p_{0}$ & $\tau$ & $m=10$ & $m=20$ & $m=50$ & $m=100$ & $m=200$ & $m=1000$ & $m \rightarrow \infty$ \\
\hline \multicolumn{9}{|c|}{$n=25$} \\
\hline \multirow[t]{8}{*}{0.01} & 1.1 & $(3.00,220.8,2538.9)$ & $(3.00,133.5,504.5)$ & $(3.00,105.4,235.3)$ & $(3.00,70.1,173.3)$ & $(3.00,44.6,102.1)$ & $(3.00,33.0,32.5)$ & $(3.00,33.0,32.5)$ \\
\hline & & $00,208.2,2204.1)$ & $.10,128.2,477.9)$ & $3.12,101.8,225.7)$ & $(3.04,68.1,166.0)$ & $(3.00,43.8,98.2)$ & $(3.13,33.1,37.5)$ & $(3.00,32.6,32.1)$ \\
\hline & 1.2 & $00,166.1,1630.9)$ & $(3.00,104.9,367.2)$ & $(3.00,84.1,183.0)$ & $(3.00,56.8,135.0)$ & $(3.00,37.1,80.0)$ & $(3.00,28.1,27.6)$ & $(3.00,28.1,27.6)$ \\
\hline & & $3.00,157.9,1447.2)$ & $(3.10,101.3,349.9)$ & $(3.12,81.5,176.3)$ & $(3.04,55.4,129.9)$ & $(3.00,36.5,77.4)$ & $(3.13,28.2,31.2)$ & $(3.00,27.8,27.3)$ \\
\hline & 1.5 & $(3.00,82.5,558.8)$ & $(3.00,57.6,167.0)$ & $(3.00,47.8,96.8)$ & $(3.00,33.7,71.8)$ & $(3.00,23.5,43.5)$ & $(3.00,18.8,18.3)$ & $(3.00,18.8,18.2)$ \\
\hline & & $(3.00,79.7,517.7)$ & $(3.10,56.2,161.2)$ & $(3.12,46.8,94.2)$ & $(3.04,33.1,69.8)$ & $(3.00,23.2,42.5)$ & $(3.13,18.9,19.8)$ & $(3.00,18.7,18.1)$ \\
\hline & 2.0 & $(3.00,35.7,159.5)$ & $(3.00,27.8,64.2)$ & $(3.00,24.0,43.6)$ & $(3.00,17.8,32.7)$ & & $(3.00,11.4,10.8)$ & $(3.00,11.4,10.8)$ \\
\hline & & $(3.00,35.0,153.0)$ & $(3.10,27.4,62.8)$ & $(3.12,23.7,42.8)$ & $(3.04,17.7,32.1)$ & $(3.00,13.3,20.3)$ & $(3.13,11.4,11.4)$ & $(3.00,11.3,10.8)$ \\
\hline \multirow[t]{8}{*}{0.05} & 1.1 & $(3.00,278.8,1340.8)$ & $(3.00,166.0,381.4)$ & $(3.00,124.6,208.6)$ & $(3.00,104.1,136.2)$ & $(3.00,97.2,101.0)$ & $(3.00,96.3,95.7)$ & $(3.00,96.3,95.7)$ \\
\hline & & $00,280.3,1494$ & $.03,166.9,420.1)$ & $.01,121.7,202.7)$ & $(3.01,104.0,14$ & $(3.05,97.3,108$ & & \\
\hline & 1.2 & $(3.00,176.0,729.5)$ & $(3.00,110.4,235.6)$ & $(3.00,85.1,135.5)$ & $(3.00,72.5,91.0)$ & $(3.00,68.2,70.1)$ & $(3.00,67.6,67.1)$ & $(3.00,67.6,67.1)$ \\
\hline & & $(3.00,176.8,816.0)$ & $(3.03,111.0,257.4)$ & $(3.01,83.5,132.2)$ & $(3.01,72.5,93.6)$ & $(3.05,68.3,74.5)$ & $(3.25,67.7,71.7)$ & $(3.00,66.5,65.9)$ \\
\hline & 1.5 & $(3.00,57.8,170.6)$ & $(3.00,40.9,72.8)$ & $(3.00,33.6,47.0)$ & $(3.00,29.9,34.2)$ & $(3.00,28.6,28.7)$ & $(3.00,28.5,27.9)$ & $(3.00,28.5,27.9)$ \\
\hline & & $(3.00,57.9,188.6)$ & $(3.03,41.1,77.9)$ & $(3.01,33.2,46.3)$ & $(3.01,30.0,35.0)$ & $(3.05,28.7,29.8)$ & $(3.25,28.5,29.1)$ & $(3.00,28.1,27.6)$ \\
\hline & 2.0 & $(3.00,16.1,32.8)$ & $(3.00,13.0,18.5)$ & $(3.00,11.4,13.6)$ & $(3.00,10.6,10.9)$ & $(3.00,10.3,9.9)$ & $(3.00,10.3,9.7)$ & $(3.00,10.3,9.7)$ \\
\hline & & $(3.00,16.1,34.9)$ & $(3.03,13.0,19.3)$ & $(3.01,11.4,13.5)$ & $(3.01,10.7,11.1)$ & $(3.05,10.4,10.1)$ & $(3.25,10.3,10.0)$ & $(3.00,10.3,9.7)$ \\
\hline \multirow[t]{8}{*}{0.10} & 1.1 & $(3.00,304.5,1212.9)$ & $(3.00,190.0,407.7)$ & $(3.00,155.3,217.0)$ & $(3.00,153.9,201.3)$ & $(3.00,151.3,198.9)$ & $(3.00,146.7,195.0)$ & $(3.00,65.1,64.5)$ \\
\hline & & $.00,310.9,1211.7)$ & $.00,196.0,398.4)$ & $(3.00,159.4,221.0)$ & $(3.00,15$ & $(3.00,154.6,19$ & $(2.99,140.5,1$ & $(2.99,64.1,63.5)$ \\
\hline & 1.2 & $(3.00,166.3,575.9)$ & $(3.00,110.1,216.9)$ & $(3.00,92.6,124.7)$ & $(3.00,91.9,117.3)$ & $(3.00,90.5,115.9)$ & $(3.00,87.9,113.7)$ & $(3.00,41.8,41.2)$ \\
\hline & & $(3.00,170.9,573.2)$ & $(3.00,113.9,213.5)$ & $(3.00,95.1,127.2)$ & $(3.00,92.9,117.1)$ & $(3.00,92.6,116.6)$ & $(2.99,84.6,109.9)$ & $(2.99,41.3,40.7)$ \\
\hline & 1.5 & $(3.00,39.6,97.5)$ & $(3.00,30.0,47.9)$ & $(3.00,26.8,32.8)$ & $(3.00,26.8,31.7)$ & $(3.00,26.4,31.4)$ & $(3.00,25.8,30.8)$ & $(3.00,14.5,14.0)$ \\
\hline & & & $(3.00,31.1,47.9)$ & $(3.00,27.6,33.6)$ & $(3.00,27.1,31.9)$ & $(3.00,27.0,3$ & $(2.99,25.1,30.1)$ & $(2.99,14.4,13.9)$ \\
\hline & 2.0 & $(3.00,8.4,13.5)$ & $(3.00,7$ & & & & & $(3.00,4.6,4.0)$ \\
\hline & & $(3.00,8.7,13.6)$ & $(3.00,7.5,9.0)$ & $(3.00,7.0,7.4)$ & $(3.00,7.0,7.2)$ & $(3.00,6.9,7.2)$ & $(2.99,6.6,6.8)$ & $(2.99,4.6,4.0)$ \\
\hline \multirow[t]{8}{*}{0.20} & 1.1 & $(3.00,270.7,635.9)$ & $(3.00,219.4,401.3)$ & $(3.00,186.5,252.8)$ & $(3.00,183.0,229.7)$ & $(3.00,180.7,227.1)$ & $(3.00,173.3,220.8)$ & $(3.00,87.3,86.7)$ \\
\hline & & & & $8)$ & $(3.00,1$ & 6.4) & $(3.00$, & $(2.99,85.9,85.3)$ \\
\hline & 1.2 & $(3.00,126.2,269.5)$ & $(3.00,1$ & $(3.00,89$ & $(3.00,88$ & $(3.00,87.5,106.8)$ & $(3.00,8$ & $(3.00,45.7,45.1)$ \\
\hline & & $(2.99,123.8,263.1)$ & & $(3.00,90.8,116.1)$ & $(3.00,88.8,1$ & $(3.00,88.5,106.8)$ & $(3.00,8$ & $(2.99,45.1,44.6)$ \\
\hline & 1.5 & 6) & 4) & $(3.0$ & $(3.00$ & $(3.00,16$ & $(3.00$ & $(3.00,10.3,9.8)$ \\
\hline & & 2) & $(3$ & $(3.0$ & $(3.00,1$ & $(3.00,1$ & $(3.00,1$ & $(2.99,10.3,9.7)$ \\
\hline & 2.0 & $3.8)$ & $(3.00,3$ & $(3.00$, & $(3.00,3$ & $(3.00$ & $(3.00,3.0$ & $(3.00,2.5,1.8)$ \\
\hline & & $(2.99,3.4,3.8)$ & $(3.00,3.3,3.1)$ & $(3.00,3.2,2.8)$ & $(3.00,3.1,2.7)$ & $(3.00,3.1,2.7)$ & & \\
\hline \multicolumn{9}{|c|}{$n=50$} \\
\hline \multirow[t]{8}{*}{0.01} & 1.1 & $(3.00,217.3,1748.7)$ & $(3.00,146.2,392.8)$ & $(3.00,98.8,204.4)$ & $(3.00,71.2,125.0)$ & $(3.00,59.9,69.6)$ & $(3.00,58.4,57.8)$ & $(3.00,58.4,57.8)$ \\
\hline & & & & )) & $(3.01$ & & $(3.29$, & $(3.00,56.3$, \\
\hline & 1.2 & $(3.0$ & $(3.0$ & $3.00,7$ & $(3.00$, & 3.6) & $(3.00,4$ & $(3.00,46.3,45.8)$ \\
\hline & & $(3$. & $(3$. & $3047-7-2$ & $(3.01$ & $9.2)$ & $(3.29$, & $(3.00,44.9,44.4)$ \\
\hline & 1.5 & $66.9,316.6)$ & & $(3.0$ & $(3.00$ & $(3.0$ & $(3.00,26.1$, & $(3.00,26.1,25.5)$ \\
\hline & & $63.7,287.3)$ & , 123.8) & & $(3.01$, & & $(3.29,26.2$, & $(3.00,25.5,25.0)$ \\
\hline & 2.0 & $(3.00,2$ & $(3$ & $(3.0$ & $(3.0)$ & $(3.0$ & $(3.00$ & $(3.00,13.0,12.4)$ \\
\hline & & & $(3.11,21.5,41.1)$ & $(3.04,16.9,24.8)$ & $(3.01,14.6,18.7)$ & $(3.03,13.2,13.8)$ & $(3.29,13.0,13.3)$ & $(3.00,12.8,12.2)$ \\
\hline \multirow[t]{8}{*}{0.05} & 1.1 & $(3.00,272.8,1082.1)$ & $(3.00,178.5,353.0)$ & $3.00,138.4,191.9)$ & $(3.00,138.3,165.8)$ & $(3.00,144.0,167.4)$ & $(3.00,167.8,178$ & $(3.00,185.4,184.9)$ \\
\hline & & & & 01 & (3.01, & $(3.01$ & $(3.01$ & $(3.6)$ \\
\hline & 1.2 & & $4.4,190$. & $(3.00,83$. & $(3.00,83.8,98$. & $(3.00,8$ & $(3.00,100.8,10$ & $, 110.9,11$ \\
\hline & & & & $(3.01,84.0,111.3$ & $(3.01,84.3,97.7$ & $(3.01,88.4,98.4)$ & $(3.01,102.0,104.5)$ & $(3.00,106.7,106.1)$ \\
\hline & 1.5 & $(3.00,37.4,87.2)$ & $(3.00,29.3,44.3)$ & $(3.00,25.2,30.2)$ & $(3.00,25.4,28.3)$ & $(3.00,26.3,28.7)$ & $(3.00,29.8,30.7)$ & $(3.00,32.4,31.9)$ \\
\hline & & $(3.04,36.7,84.2)$ & $(3.04,30.3,47.0)$ & $(3.01,25.5,30.5)$ & $(3.01,25.8,28.4)$ & $(3.01,26.9,28.8)$ & $(3.01,30.5,30.6)$ & $(3.00,31.7,31.1)$ \\
\hline & 2.0 & $(3.00,8.4,13.0)$ & $(3.00,7.4,8.8)$ & $(3.00,6.8,7.0)$ & $(3.00,6.9,6.9)$ & $(3.00,7.1,7.0)$ & $(3.00,7.8,7.5)$ & $(3.00,8.3,7.7)$ \\
\hline & & $(3.04,8.4,12.8)$ & $(3.04,7.6,9.2)$ & $(3.01,6.9,7.2)$ & $(3.01,7.0,7.0)$ & $(3.01,7.3,7.1)$ & $(3.01,8.0,7.5)$ & $(3.00,8.2,7.7)$ \\
\hline \multirow[t]{8}{*}{0.10} & 1.1 & $(3.00,266.5,779.9)$ & $185.5,341.0)$ & $3.00,149.6,199$ & $(3.00,141.6,161$ & $(3.00,143.5,148.5)$ & $(3.00,150.0,149.5)$ & $(3.00,150.1,149.5)$ \\
\hline & & $.02,268.4,778.9)$ & $(3.01,185.7,336.8)$ & $(3.00,148.2,197.6)$ & $(3.01,141.9,163.7)$ & $(3.04,144.2,150.3)$ & $(3.20,150.8,161.7)$ & $(3.00,144.1,143.5)$ \\
\hline & 1.2 & $(3.00,122.2,311.0)$ & $(3.00,90.2,151.1)$ & $(3.00,75.3,95.7)$ & $(3.00,72.1,79.9)$ & $(3.00,73.2,75.1)$ & $(3.00,76.4,75.8)$ & $(3.00,76.4,75.9)$ \\
\hline & & $(3.02,123.9,311.5)$ & $(3.01,90.8,150.6)$ & $(3.00,75.0,95.2)$ & $(3.01,72.6,81.3)$ & $(3.04,73.9,76.1)$ & $(3.20,77.0,80.9)$ & $(3.00,74.0,73.4)$ \\
\hline & 1.5 & $(3.00,20.9,38.4)$ & $(3.00,17.5,23.4)$ & $(3.00,15.7,17.6)$ & $(3.00,15.4,15.8)$ & $(3.00,15.7,15.4)$ & $(3.00,16.2,15.7)$ & $(3.00,16.2,15.7)$ \\
\hline & & $(3.02,21.4,38.2)$ & $(3.01,17.7,23.7)$ & $(3.00,15.8,17.7)$ & $(3.01,15.6,16.1)$ & $(3.04,15.9,15.7)$ & $(3.20,16.4,16.3)$ & $(3.00,16.0,15.4)$ \\
\hline & 2.0 & $(3.00,3.8,4.3)$ & $(3.00,3.6,3.4)$ & $(3.00,3.4,3.0)$ & $(3.00,3.4,2.9)$ & $(3.00,3.4,2.9)$ & $(3.00,3.5,2.9)$ & $(3.00,3.5,2.9)$ \\
\hline & & $(3.02,3.9,4.4)$ & $(3.01,3.6,3.5)$ & $(3.00,3.4,3.0)$ & $(3.01,3.4,2.9)$ & $(3.04,3.5,2.9)$ & $(3.20,3.6,3.0)$ & $(3.00,3.5,2.9)$ \\
\hline \multirow[t]{8}{*}{0.20} & 1.1 & $(3.00,189.4,314.8)$ & $.00,165.1,241.4)$ & $(3.00,146.4,181.7)$ & $(3.00,140.0,159.1)$ & $(3.00,136.5,142.3)$ & $(3.00,136.6,136.1)$ & $(3.00,136.6,136.1)$ \\
\hline & & & $(2.99,159.2,231.9)$ & $(3.00,147.9,183.9)$ & $(3.00,139.2,159.3)$ & $(3.02,137.7,147.3)$ & $(3.11,139.5,148.4)$ & $(3.00,131.7,131.1)$ \\
\hline & 1.2 & $(3.00,80.6,148.4)$ & $(3.00,65.6,97.1)$ & $(3.00,57.3,68.9)$ & $(3.00,54.8,60.4)$ & $(3.00,53.7,55.0)$ & $(3.00,53.8,53.3)$ & $(3.00,53.8,53.3)$ \\
\hline & & $(2.99,78.0,142.6)$ & $(2.99,63.7,93.9)$ & $(3.00,57.9,69.6)$ & $(3.00,54.8,60.6)$ & $(3.02,54.4,56.8)$ & $(3.11,55.0,57.2)$ & $(3.00,52.4,51.8)$ \\
\hline & 1.5 & $(3.00,8.9,12.8)$ & $(3.00,7.9,9.1)$ & $(3.00,7.4,7.5)$ & $(3.00,7.2,7.0)$ & $(3.00,7.2,6.7)$ & $(3.00,7.2,6.7)$ & $(3.00,7.2,6.7)$ \\
\hline & & $(2.99,8.8,12.7)$ & $(2.99,7.8,9.0)$ & $(3.00,7.5,7.6)$ & $(3.00,7.3,7.1)$ & $(3.02,7.3,6.9)$ & $(3.11,7.4,6.9)$ & $(3.00,7.2,6.6)$ \\
\hline & 2.0 & $(3.00,1.6,1.1)$ & $(3.00,1.6,1.0)$ & $(3.00,1.6,0.9)$ & $(3.00,1.6,0.9)$ & $(3.00,1.5,0.9)$ & $(3.00,1.6,0.9)$ & $(3.00,1.6,0.9)$ \\
\hline & & $(2.99,1.6,1.1)$ & $(2.99,1.6,1.0)$ & $(3.00,1.6,0.9)$ & $(3.00,1.6,0.9)$ & $(3.02,1.6,0.9)$ & $(3.11,1.6,0.9)$ & $(3.00,1.6,0.9)$ \\
\hline
\end{tabular}


Table 25: $\left(K, \mathrm{ARL}_{1}, \mathrm{SDRL}_{1}\right)$ for the hypergeometric $n p$ chart and $\left(K^{\prime \prime}, \mathrm{ARL}_{1}, \mathrm{SDRL}_{1}\right)$ for the binomial $n p$ chart with $N=10000, n \in\{25,50\}, p_{0} \in\{0.01,0.05,0.10,0.20\}, m \in\{10,20,50,100,200,1000, \infty\}$

\begin{tabular}{|c|c|c|c|c|c|c|c|c|}
\hline$p_{0}$ & $\tau$ & $m=10$ & $m=20$ & $m=50$ & $m=100$ & $m=200$ & $m=1000$ & $m \rightarrow \infty$ \\
\hline \multicolumn{9}{|c|}{$n=25$} \\
\hline 0.01 & 1.2 & $\begin{array}{c}(3.00,214.3,2361.3) \\
(3.00,208.2,2204.1) \\
(3.00,161.9,1534.2) \\
(3.00,157.9,1447.2) \\
(3.00,81.1,537.5) \\
(3.00,79.7,517.7) \\
(3.00,35.3,156.2) \\
(3.00,35.0,153.0)\end{array}$ & $\begin{array}{c}(3.00,130.8,486.2) \\
(3.10,128.2,477.9) \\
(3.00,103.1,355.6) \\
(3.10,101.3,349.9) \\
(3.00,56.9,163.3) \\
(3.10,56.2,161.2) \\
(3.00,27.6,63.4) \\
(3.10,27.4,62.8)\end{array}$ & $\begin{array}{c}(3.00,103.5,230.1) \\
(3.12,101.8,225.7) \\
(3.00,82.8,179.3) \\
(3.12,81.5,176.3) \\
(3.00,47.3,95.4) \\
(3.12,46.8,94.2) \\
(3.00,23.8,43.1) \\
(3.12,23.7,42.8)\end{array}$ & $\begin{array}{c}(3.00,69.1,169.6) \\
(3.04,68.1,166.0) \\
(3.00,56.1,132.4) \\
(3.04,55.4,129.9) \\
(3.00,33.4,70.8) \\
(3.04,33.1,69.8) \\
(3.00,17.8,32.4) \\
(3.04,17.7,32.1)\end{array}$ & $\begin{array}{c}(3.00,44.2,100.1) \\
(3.00,43.8,98.2) \\
(3.00,36.8,78.7) \\
(3.00,36.5,77.4) \\
(3.00,23.3,43.0) \\
(3.00,23.2,42.5) \\
(3.00,13.4,20.4) \\
(3.00,13.3,20.3)\end{array}$ & $\begin{array}{l}(3.00,32.8,32.3) \\
(3.10,32.8,34.1) \\
(3.00,28.0,27.5) \\
(3.10,28.0,28.8) \\
(3.00,18.7,18.2) \\
(3.10,18.7,18.7) \\
(3.00,11.4,10.8) \\
(3.10,11.4,11.0)\end{array}$ & $\begin{array}{l}(3.00,32.8,32.3) \\
(3.00,32.6,32.1) \\
(3.00,28.0,27.4) \\
(3.00,27.8,27.3) \\
(3.00,18.7,18.2) \\
(3.00,18.7,18.1) \\
(3.00,11.4,10.8) \\
(3.00,11.3,10.8)\end{array}$ \\
\hline 0.05 & 1.2 & $\begin{array}{c}(3.00,274.1,1304.4) \\
(2.99,269.5,1269.6) \\
(3.00,173.5,713.2) \\
(2.99,171.1,697.4) \\
(3.00,57.3,168.3) \\
(2.99,56.8,166.1) \\
(3.00,16.1,32.6) \\
(2.99,16.0,32.4)\end{array}$ & $\begin{array}{c}(3.00,163.8,374.5) \\
(3.00,161.6,371.2) \\
(3.00,109.1,231.9) \\
(3.00,107.9,230.0) \\
(3.00,40.6,72.1) \\
(3.00,40.3,71.7) \\
(3.00,12.9,18.4) \\
(3.00,12.9,18.3)\end{array}$ & $\begin{array}{c}(3.00,123.1,205.5) \\
(3.01,121.7,202.7) \\
(3.00,84.3,133.7) \\
(3.01,83.5,132.2) \\
(3.00,33.4,46.7) \\
(3.01,33.2,46.3) \\
(3.00,11.4,13.5) \\
(3.01,11.4,13.5)\end{array}$ & $\begin{array}{c}(3.00,103.0,134.5) \\
(3.01,104.0,140.2) \\
(3.00,71.9,90.1) \\
(3.01,72.5,93.6) \\
(3.00,29.7,34.0) \\
(3.01,30.0,35.0) \\
(3.00,10.6,10.9) \\
(3.01,10.7,11.1)\end{array}$ & $\begin{array}{c}(3.00,96.2,100.0) \\
(3.03,96.4,104.3) \\
(3.00,67.6,69.5) \\
(3.03,67.8,72.1) \\
(3.00,28.5,28.5) \\
(3.03,28.5,29.2) \\
(3.00,10.3,9.9) \\
(3.03,10.3,10.0)\end{array}$ & $\begin{array}{c}(3.00,95.3,94.8) \\
(3.23,95.3,98.4) \\
(3.00,67.1,66.5) \\
(3.23,67.1,68.6) \\
(3.00,28.3,27.7) \\
(3.23,28.3,28.3) \\
(3.00,10.3,9.7) \\
(3.23,10.3,9.8)\end{array}$ & $\begin{array}{c}(3.00,95.3,94.8) \\
(3.00,94.4,93.8) \\
(3.00,67.1,66.5) \\
(3.00,66.5,65.9) \\
(3.00,28.3,27.7) \\
(3.00,28.1,27.6) \\
(3.00,10.3,9.7) \\
(3.00,10.3,9.7)\end{array}$ \\
\hline 0.10 & 1.1 & $\begin{array}{c}(3.00,300.1,1188.4) \\
(2.99,295.8,1164.6) \\
(3.00,164.3,566.4) \\
(2.99,162.3,557.0) \\
(3.00,39.3,96.6) \\
(2.99,39.1,95.7) \\
(3.00,8.4,13.5) \\
(2.99,8.3,13.4) \\
\end{array}$ & $\begin{array}{c}(3.00,187.7,401.4) \\
(2.99,185.4,395.3) \\
(3.00,109.0,214.2) \\
(2.99,107.9,211.5) \\
(3.00,29.8,47.6) \\
(2.99,29.6,47.2) \\
(3.00,7.2,8.8) \\
(2.99,7.2,8.8) \\
\end{array}$ & $\begin{array}{c}(3.00,154.4,219.1) \\
(2.99,151.8,211.6) \\
(3.00,92.1,125.7) \\
(2.99,90.8,122.1) \\
(3.00,26.8,33.0) \\
(2.99,26.5,32.4) \\
(3.00,6.9,7.2) \\
(2.99,6.8,7.2) \\
\end{array}$ & $\begin{array}{c}(3.00,152.2,198.9) \\
(2.99,150.4,196.3) \\
(3.00,91.1,116.1) \\
(2.99,90.2,114.9) \\
(3.00,26.6,31.5) \\
(2.99,26.5,31.3) \\
(3.00,6.8,7.1) \\
(2.99,6.8,7.1) \\
\end{array}$ & $\begin{array}{c}(3.00,153.0,199.1) \\
(3.00,154.6,199.2) \\
(3.00,91.5,116.3) \\
(3.00,92.6,116.6) \\
(3.00,26.7,31.6) \\
(3.00,27.0,31.8) \\
(3.00,6.9,7.1) \\
(3.00,6.9,7.2) \\
\end{array}$ & $\begin{array}{c}(3.00,149.6,196.4) \\
(3.00,154.0,198.7) \\
(3.00,89.6,114.7) \\
(3.00,92.2,116.3) \\
(3.00,26.3,31.2) \\
(3.00,27.0,31.7) \\
(3.00,6.8,7.0) \\
(3.00,6.9,7.1) \\
\end{array}$ & $\begin{array}{c}(3.00,64.6,64.0) \\
(2.99,64.1,63.5) \\
(3.00,41.5,41.0) \\
(2.99,41.3,40.7) \\
(3.00,14.5,13.9) \\
(2.99,14.4,13.9) \\
(3.00,4.6,4.0) \\
(2.99,4.6,4.0)\end{array}$ \\
\hline 0.20 & 1.1 & $\begin{array}{c}(3.00,279.1,708.9) \\
(3.00,287.7,699.2) \\
(3.00,128.2,291.6) \\
(3.00,132.4,288.9) \\
(3.00,20.9,39.5) \\
(3.00,21.6,39.5) \\
(3.00,3.4,3.8) \\
(3.00,3.5,3.8) \\
\end{array}$ & $\begin{array}{c}(3.00,218.4,414.4) \\
(2.99,214.6,390.9) \\
(3.00,102.6,177.5) \\
(2.99,101.2,169.9) \\
(3.00,17.9,24.6) \\
(2.99,17.8,24.2) \\
(3.00,3.2,3.0) \\
(2.99,3.2,3.0) \\
\end{array}$ & $\begin{array}{c}(3.00,184.5,249.9) \\
(3.00,188.0,250.8) \\
(3.00,89.1,115.3) \\
(3.00,90.8,116.1) \\
(3.00,16.5,18.8) \\
(3.00,16.8,19.1) \\
(3.00,3.1,2.7) \\
(3.00,3.2,2.8) \\
\end{array}$ & $\begin{array}{c}(3.00,181.0,227.1) \\
(3.00,183.0,227.7) \\
(3.00,87.8,106.9) \\
(3.00,88.8,107.4) \\
(3.00,16.4,18.1) \\
(3.00,16.6,18.3) \\
(3.00,3.1,2.7) \\
(3.00,3.1,2.7) \\
\end{array}$ & $\begin{array}{c}(3.00,181.6,226.8) \\
(3.00,182.3,226.4) \\
(3.00,88.0,106.8) \\
(3.00,88.5,106.8) \\
(3.00,16.4,18.1) \\
(3.00,16.5,18.2) \\
(3.00,3.1,2.7) \\
(3.00,3.1,2.7) \\
\end{array}$ & $\begin{array}{c}(3.00,177.6,223.5) \\
(3.00,181.7,225.9) \\
(3.00,86.2,105.3) \\
(3.00,88.2,106.6) \\
(3.00,16.2,17.9) \\
(3.00,16.5,18.2) \\
(3.00,3.1,2.7) \\
(3.00,3.1,2.7) \\
\end{array}$ & $\begin{array}{c}(3.00,86.6,86.0) \\
(2.99,85.9,85.3) \\
(3.00,45.4,44.9) \\
(2.99,45.1,44.6) \\
(3.00,10.3,9.7) \\
(2.99,10.3,9.7) \\
(3.00,2.5,1.8) \\
(2.99,2.5,1.8) \\
\end{array}$ \\
\hline & & & & & $n=50$ & & & \\
\hline 0.01 & 1.1 & $\begin{array}{c}(3.00,207.7,1592.5) \\
(3.00,198.9,1457.9) \\
(3.00,148.4,980.5) \\
(3.00,143.0,909.8) \\
(3.00,65.3,301.3) \\
(3.00,63.7,287.3) \\
(3.00,24.8,75.7) \\
(3.00,24.5,73.8)\end{array}$ & $\begin{array}{c}(3.00,141.3,377.2) \\
(3.04,143.8,443.0) \\
(3.00,105.3,262.9) \\
(3.04,107.1,306.2) \\
(3.00,50.9,108.3) \\
(3.04,51.6,122.8) \\
(3.00,21.3,37.5) \\
(3.04,21.5,40.9)\end{array}$ & $\begin{array}{c}(3.00,96.2,196.6) \\
(3.04,93.7,189.6) \\
(3.00,73.5,143.5) \\
(3.04,71.9,139.0) \\
(3.00,37.8,65.4) \\
(3.04,37.3,64.0) \\
(3.00,17.1,25.1) \\
(3.04,16.9,24.8)\end{array}$ & $\begin{array}{c}(3.00,69.7,120.8) \\
(3.00,68.3,116.9) \\
(3.00,54.5,89.4) \\
(3.00,53.6,86.9) \\
(3.00,29.7,42.5) \\
(3.00,29.3,41.8) \\
(3.00,14.2,17.5) \\
(3.00,14.1,17.3)\end{array}$ & $\begin{array}{l}(3.00,59.3,71.2) \\
(3.03,59.6,77.6) \\
(3.00,47.1,54.7) \\
(3.03,47.3,59.2) \\
(3.00,26.4,28.8) \\
(3.03,26.6,30.5) \\
(3.00,13.1,13.3) \\
(3.03,13.2,13.8)\end{array}$ & $\begin{array}{l}(3.00,57.3,56.8) \\
(3.27,57.5,64.0) \\
(3.00,45.6,45.1) \\
(3.27,45.7,49.9) \\
(3.00,25.8,25.2) \\
(3.27,25.9,27.0) \\
(3.00,12.9,12.3) \\
(3.27,12.9,12.8)\end{array}$ & $\begin{array}{l}(3.00,57.3,56.8) \\
(3.00,56.3,55.8) \\
(3.00,45.6,45.1) \\
(3.00,44.9,44.4) \\
(3.00,25.8,25.2) \\
(3.00,25.5,25.0) \\
(3.00,12.9,12.3) \\
(3.00,12.8,12.2)\end{array}$ \\
\hline 0.05 & 1.2 & $\begin{array}{c}(3.00,264.9,1035.9) \\
(3.04,258.7,1008.8) \\
(3.00,147.1,491.7) \\
(3.04,144.6,480.0) \\
(3.00,36.9,85.6) \\
(3.04,36.7,84.2) \\
(3.00,8.4,12.9) \\
(3.04,8.4,12.8) \\
\end{array}$ & $\begin{array}{c}(3.00,174.2,342.1) \\
(3.02,171.8,351.4) \\
(3.00,102.3,186.0) \\
(3.02,101.1,190.3) \\
(3.00,29.0,43.7) \\
(3.02,28.8,44.3) \\
(3.00,7.4,8.8) \\
(3.02,7.4,8.8)\end{array}$ & $\begin{array}{c}(3.00,135.3,187.2) \\
(3.00,138.5,191.3) \\
(3.00,82.0,108.8) \\
(3.00,84.0,111.3) \\
(3.00,24.9,29.8) \\
(3.00,25.5,30.5) \\
(3.00,6.8,7.0) \\
(3.00,6.9,7.2)\end{array}$ & $\begin{array}{c}(3.00,135.3,162.4) \\
(3.00,135.4,160.9) \\
(3.00,82.3,96.9) \\
(3.00,82.6,96.3) \\
(3.00,25.2,28.0) \\
(3.00,25.4,28.0) \\
(3.00,6.9,6.9) \\
(3.00,6.9,6.9)\end{array}$ & $\begin{array}{c}(3.00,143.5,162.8) \\
(3.00,86.7,98.5) \\
(3.00,87.3,97.8) \\
(3.00,26.3,28.6) \\
(3.00,26.6,28.6) \\
(3.00,7.1,7.0) \\
(3.00,7.2,7.1)\end{array}$ & $\begin{array}{c}(3.01,168.8,173.8) \\
(3.00,100.7,105.3) \\
(3.01,102.0,104.5) \\
(3.00,29.9,30.6) \\
(3.01,30.5,30.6) \\
(3.00,7.8,7.5) \\
(3.01,8.0,7.5)\end{array}$ & $\begin{array}{c}(3.00,181.1,180.6) \\
(3.00,176.9,176.4) \\
(3.00,108.8,108.2) \\
(3.00,106.7,106.1) \\
(3.00,32.1,31.5) \\
(3.00,31.7,31.1) \\
(3.00,8.3,7.7) \\
(3.00,8.2,7.7)\end{array}$ \\
\hline 0.10 & 1.2 & $\begin{array}{c}(3.00,259.7,753.8) \\
(3.00,254.4,757.1) \\
(3.00,119.8,302.8) \\
(3.00,117.8,303.5) \\
(3.00,20.7,37.9) \\
(3.00,20.6,37.6) \\
(3.00,3.8,4.3) \\
(3.00,3.8,4.3)\end{array}$ & $\begin{array}{c}(3.00,181.3,331.9) \\
(3.01,185.7,336.8) \\
(3.00,88.6,148.0) \\
(3.01,90.8,150.6) \\
(3.00,17.3,23.1) \\
(3.01,17.7,23.7) \\
(3.00,3.6,3.4) \\
(3.01,3.6,3.5)\end{array}$ & $\begin{array}{c}(3.00,148.4,195.8) \\
(3.00,148.2,197.6) \\
(3.00,75.0,94.3) \\
(3.00,75.0,95.2) \\
(3.00,15.7,17.5) \\
(3.00,15.8,17.7) \\
(3.00,3.4,3.0) \\
(3.00,3.4,3.0)\end{array}$ & $\begin{array}{c}(3.00,140.5,160.0) \\
(3.01,141.9,163.7) \\
(3.00,71.8,79.5) \\
(3.01,72.6,81.3) \\
(3.00,15.4,15.8) \\
(3.01,15.6,16.1) \\
(3.00,3.4,2.9) \\
(3.01,3.4,2.9)\end{array}$ & $\begin{array}{c}(3.00,141.6,146.2) \\
(3.02,141.9,146.6) \\
(3.00,72.5,74.2) \\
(3.02,72.9,74.5) \\
(3.00,15.6,15.4) \\
(3.02,15.8,15.5) \\
(3.00,3.4,2.9) \\
(3.02,3.5,2.9)\end{array}$ & $\begin{array}{c}(3.00,147.0,146.5) \\
(3.18,146.5,150.4) \\
(3.00,75.2,74.6) \\
(3.18,75.1,76.3) \\
(3.00,16.1,15.5) \\
(3.18,16.1,15.7) \\
(3.00,3.5,2.9) \\
(3.18,3.5,2.9)\end{array}$ & $\begin{array}{c}(3.00,147.0,146.5) \\
(3.00,144.1,143.5) \\
(3.00,75.2,74.6) \\
(3.00,74.0,73.4) \\
(3.00,16.1,15.5) \\
(3.00,16.0,15.4) \\
(3.00,3.5,2.9) \\
(3.00,3.5,2.9)\end{array}$ \\
\hline 0.20 & 1.1 & $\begin{array}{c}(3.00,185.4,307.1) \\
(2.99,181.5,299.7) \\
(3.00,79.3,145.5) \\
(2.99,78.0,142.6) \\
(3.00,8.8,12.7) \\
(2.99,8.8,12.7) \\
(3.00,1.6,1.1) \\
(2.99,1.6,1.1)\end{array}$ & $\begin{array}{c}(3.00,164.6,238.3) \\
(3.00,166.7,239.5) \\
(3.00,65.5,95.9) \\
(3.00,66.2,96.0) \\
(3.00,7.9,9.0) \\
(3.00,8.0,9.1) \\
(3.00,1.6,1.0) \\
(3.00,1.6,1.0)\end{array}$ & $\begin{array}{c}(3.00,147.2,182.8) \\
(3.00,147.9,183.9) \\
(3.00,57.6,69.1) \\
(3.00,57.9,69.6) \\
(3.00,7.4,7.5) \\
(3.00,7.5,7.6) \\
(3.00,1.6,0.9) \\
(3.00,1.6,0.9)\end{array}$ & $\begin{array}{c}(3.00,140.3,160.2) \\
(3.00,139.2,159.3) \\
(3.00,55.0,60.8) \\
(3.00,54.8,60.6) \\
(3.00,7.3,7.1) \\
(3.00,7.3,7.1) \\
(3.00,1.6,0.9) \\
(3.00,1.6,0.9)\end{array}$ & $\begin{array}{c}(3.00,135.2,141.3) \\
(3.01,135.5,143.3) \\
(3.00,53.4,54.8) \\
(3.01,53.6,55.6) \\
(3.00,7.2,6.7) \\
(3.01,7.2,6.8) \\
(3.00,1.6,0.9) \\
(3.01,1.6,0.9)\end{array}$ & $\begin{array}{c}(3.00,134.1,133.5) \\
(3.09,134.2,137.1) \\
(3.00,53.1,52.5) \\
(3.09,53.3,53.7) \\
(3.00,7.2,6.6) \\
(3.09,7.2,6.7) \\
(3.00,1.6,0.9) \\
(3.09,1.6,0.9)\end{array}$ & $\begin{array}{c}(3.00,134.1,133.5) \\
(3.00,131.7,131.1) \\
(3.00,53.1,52.5) \\
(3.00,52.4,51.8) \\
(3.00,7.2,6.6) \\
(3.00,7.2,6.6) \\
(3.00,1.6,0.9) \\
(3.00,1.6,0.9)\end{array}$ \\
\hline
\end{tabular}


Table 26: $\left(\mathrm{ARL}_{1}, \mathrm{SDRL}_{1}\right)$ for the binomial $n p$ chart with $n \in\{25,50\}, p_{0} \in\{0.01,0.05,0.10,0.20\}, m \in$ $\{10,20,50,100,200,1000, \infty\}$

\begin{tabular}{|c|c|c|c|c|c|c|c|c|}
\hline$p_{0}$ & $\tau$ & $m=10$ & $m=20$ & $m=50$ & $m=100$ & $m=200$ & $m=1000$ & $m \rightarrow \infty$ \\
\hline \multicolumn{9}{|c|}{$n=25$} \\
\hline 0.01 & $\begin{array}{l}1.1 \\
1.2 \\
1.5 \\
2.0\end{array}$ & $\begin{array}{c}(208.2,2204.1) \\
(157.9,1447.2) \\
(79.7,517.7) \\
(35.0,153.0)\end{array}$ & $\begin{array}{c}(128.2,469.0) \\
(101.3,344.6) \\
(56.2,159.9) \\
(27.4,62.6)\end{array}$ & $\begin{array}{c}(101.8,225.0) \\
(81.5,175.8) \\
(46.8,94.0) \\
(23.7,42.7)\end{array}$ & $\begin{array}{c}(68.1,166.0) \\
(55.4,129.9) \\
(33.1,69.8) \\
(17.7,32.1)\end{array}$ & $\begin{array}{l}(43.8,98.2) \\
(36.5,77.4) \\
(23.2,42.5) \\
(13.3,20.3)\end{array}$ & $\begin{array}{l}(32.6,32.1) \\
(27.8,27.3) \\
(18.7,18.1) \\
(11.3,10.8)\end{array}$ & $\begin{array}{l}(32.6,32.1) \\
(27.8,27.3) \\
(18.7,18.1) \\
(11.3,10.8)\end{array}$ \\
\hline 0.05 & $\begin{array}{l}1.1 \\
1.2 \\
1.5 \\
2.0 \\
\end{array}$ & $\begin{array}{c}(280.3,1494.1) \\
(176.8,816.0) \\
(57.9,188.6) \\
(16.1,34.9) \\
\end{array}$ & $\begin{array}{c}(161.6,371.2) \\
(107.9,230.0) \\
(40.3,71.7) \\
(12.9,18.3) \\
\end{array}$ & $\begin{array}{c}(121.7,202.5) \\
(83.5,132.0) \\
(33.2,46.3) \\
(11.4,13.5) \\
\end{array}$ & $\begin{array}{c}(102.0,132.8) \\
(71.2,89.1) \\
(29.6,33.8) \\
(10.6,10.9) \\
\end{array}$ & $\begin{array}{c}(95.2,99.0) \\
(67.0,68.9) \\
(28.3,28.3) \\
(10.3,9.8) \\
\end{array}$ & $\begin{array}{c}(94.4,93.8) \\
(66.5,65.9) \\
(28.1,27.6) \\
(10.3,9.7) \\
\end{array}$ & $\begin{array}{c}(94.4,93.8) \\
(66.5,65.9) \\
(28.1,27.6) \\
(10.3,9.7) \\
\end{array}$ \\
\hline 0.10 & $\begin{array}{l}1.1 \\
1.2 \\
1.5 \\
2.0\end{array}$ & $\begin{array}{c}(310.9,1211.7) \\
(170.9,573.2) \\
(41.2,96.9) \\
(8.7,13.6) \\
\end{array}$ & $\begin{array}{c}(196.0,398.4) \\
(113.9,213.5) \\
(31.1,47.9) \\
(7.5,9.0) \\
\end{array}$ & $\begin{array}{c}(159.4,221.0) \\
(95.1,127.2) \\
(27.6,33.6) \\
(7.0,7.4) \\
\end{array}$ & $\begin{array}{c}(155.2,200.1) \\
(92.9,117.1) \\
(27.1,31.9) \\
(7.0,7.2) \\
\end{array}$ & $\begin{array}{c}(154.6,199.2) \\
(92.6,116.6) \\
(27.0,31.8) \\
(6.9,7.2) \\
\end{array}$ & $\begin{array}{c}(154.0,198.7) \\
(92.2,116.3) \\
(27.0,31.7) \\
(6.9,7.1) \\
\end{array}$ & $\begin{array}{c}(242.7,242.2) \\
(142.5,142.0) \\
(39.3,38.8) \\
(9.2,8.6)\end{array}$ \\
\hline 0.20 & $\begin{array}{l}1.1 \\
1.2 \\
1.5 \\
2.0\end{array}$ & $\begin{array}{c}(287.7,699.2) \\
(132.4,288.9) \\
(21.6,39.5) \\
(3.5,3.8) \\
\end{array}$ & $\begin{array}{c}(224.5,411.9) \\
(105.5,177.3) \\
(18.4,24.8) \\
(3.3,3.1) \\
\end{array}$ & $\begin{array}{c}(188.0,250.8) \\
(90.8,116.1) \\
(16.8,19.1) \\
(3.2,2.8) \\
\end{array}$ & $\begin{array}{c}(183.0,227.7) \\
(88.8,107.4) \\
(16.6,18.3) \\
(3.1,2.7) \\
\end{array}$ & $\begin{array}{c}(182.3,226.4) \\
(88.5,106.8) \\
(16.5,18.2) \\
(3.1,2.7) \\
\end{array}$ & $\begin{array}{c}(181.7,225.9) \\
(88.2,106.6) \\
(16.5,18.2) \\
(3.1,2.7) \\
\end{array}$ & $\begin{array}{c}(276.6,276.1) \\
(130.9,130.4) \\
(22.7,22.1) \\
(3.8,3.2) \\
\end{array}$ \\
\hline \multicolumn{9}{|c|}{$n=50$} \\
\hline 0.01 & $\begin{array}{l}1.1 \\
1.2 \\
1.5 \\
2.0 \\
\end{array}$ & $\begin{array}{c}(198.9,1457.9) \\
(143.0,909.8) \\
(63.7,287.3) \\
(24.5,73.8) \\
\end{array}$ & & $\begin{array}{c}(93.7,189.2) \\
(71.9,138.8) \\
(37.3,64.0) \\
(16.9,24.8) \\
\end{array}$ & $\begin{array}{c}(68.3,116.9) \\
(53.6,86.9) \\
(29.3,41.8) \\
(14.1,17.3) \\
\end{array}$ & & $\begin{array}{l}(56.3,55.8) \\
(44.9,44.4) \\
(25.5,25.0) \\
(12.8,12.2) \\
\end{array}$ & $\begin{array}{l}(56.3,55.8) \\
(44.9,44.4) \\
(25.5,25.0) \\
(12.8,12.2) \\
\end{array}$ \\
\hline 0.05 & $\begin{array}{l}1.1 \\
1.2 \\
1.5 \\
2.0 \\
\end{array}$ & $\begin{array}{c}(257.3,992.6) \\
(143.7,475.0) \\
(36.4,84.0) \\
(8.3,12.8)\end{array}$ & $\begin{array}{c}(170.0,333.5) \\
(100.3,182.0) \\
(28.7,43.1) \\
(7.4,8.7) \\
\end{array}$ & $\begin{array}{c}(138.5,191.3) \\
(84.0,111.3) \\
(25.5,30.5) \\
(6.9,7.2) \\
\end{array}$ & $\begin{array}{c}(135.4,160.9) \\
(82.6,96.3) \\
(25.4,28.0) \\
(6.9,6.9) \\
\end{array}$ & $\begin{array}{c}(143.5,162.8) \\
(87.3,97.8) \\
(26.6,28.6) \\
(7.2,7.1) \\
\end{array}$ & $\begin{array}{c}(165.9,172.7) \\
(100.3,103.8) \\
(30.0,30.4) \\
(7.9,7.5) \\
\end{array}$ & $\begin{array}{c}(176.9,176.4) \\
(106.7,106.1) \\
(31.7,31.1) \\
(8.2,7.7) \\
\end{array}$ \\
\hline 0.10 & $\begin{array}{l}1.1 \\
1.2 \\
1.5 \\
2.0 \\
\end{array}$ & $\begin{array}{c}(254.4,757.1) \\
(117.8,303.5) \\
(20.6,37.6) \\
(3.8,4.3)\end{array}$ & $\begin{array}{c}(177.6,323.4) \\
(87.2,144.9) \\
(17.2,22.9) \\
(3.6,3.4) \\
\end{array}$ & $\begin{array}{c}(148.2,197.6) \\
(75.0,95.2) \\
(15.8,17.7) \\
(3.4,3.0) \\
\end{array}$ & $\begin{array}{c}(139.3,159.0) \\
(71.4,79.3) \\
(15.4,15.8) \\
(3.4,2.9) \\
\end{array}$ & $\begin{array}{c}(139.6,144.0) \\
(71.8,73.4) \\
(15.6,15.3) \\
(3.4,2.9) \\
\end{array}$ & $\begin{array}{c}(144.1,143.5) \\
(74.0,73.4) \\
(16.0,15.4) \\
(3.5,2.9) \\
\end{array}$ & $\begin{array}{c}(144.1,143.5) \\
(74.0,73.4) \\
(16.0,15.4) \\
(3.5,2.9) \\
\end{array}$ \\
\hline 0.20 & $\begin{array}{l}1.1 \\
1.2 \\
1.5 \\
2.0\end{array}$ & $\begin{array}{c}(190.4,307.2) \\
(80.7,144.1) \\
(9.0,12.8) \\
(1.6,1.1)\end{array}$ & $\begin{array}{c}(166.7,239.5) \\
(66.2,96.0) \\
(8.0,9.1) \\
(1.6,1.0)\end{array}$ & $\begin{array}{c}(147.9,183.9) \\
(57.9,69.6) \\
(7.5,7.6) \\
(1.6,0.9)\end{array}$ & $\begin{array}{c}(139.2,159.3) \\
(54.8,60.6) \\
(7.3,7.1) \\
(1.6,0.9)\end{array}$ & $\begin{array}{c}(133.9,140.6) \\
(53.1,54.7) \\
(7.2,6.7) \\
(1.6,0.9)\end{array}$ & $\begin{array}{c}(131.7,131.1) \\
(52.4,51.8) \\
(7.2,6.6) \\
(1.6,0.9)\end{array}$ & $\begin{array}{c}(131.7,131.1) \\
(52.4,51.8) \\
(7.2,6.6) \\
(1.6,0.9)\end{array}$ \\
\hline
\end{tabular}

Table 27: Selected results of the simulation study regarding $\mathrm{ARL}_{1}$

\begin{tabular}{lcclccccc}
\hline$m, N, n, p_{0}, \tau$ & $\mathrm{ARL}_{1}$ & $\mathrm{ARL}_{1}^{\text {sim }}$ & $m, N, n, p_{0}, \tau$ & $\mathrm{ARL}_{1}$ & $\mathrm{ARL}_{1}^{\text {sim }}$ & $m, N, n, p_{0}, \tau$ & $\mathrm{ARL}_{1}$ & $\mathrm{ARL}_{1}^{\text {sim }}$ \\
\hline $10,100,25,0.01,1.1$ & - & - & $20,100,25,0.05,1.2$ & 260.0 & 265.1 & $50,100,50,0.1,1.5$ & 8.2 & 8.1 \\
$100,200,25,0.05,1.2$ & 106.8 & 105.2 & $200,200,50,0.1,1.5$ & 11.5 & 11.5 & $1000,200,50,0.2,2$ & 1.3 & 1.3 \\
$10,500,25,0.01,1.1$ & - & - & $20,500,25,0.05,1.2$ & 115.2 & 111.2 & $50,500,50,0.1,1.5$ & 14.8 & 14.8 \\
$100,1000,25,0.05,1.2$ & 75.2 & 75.1 & $200,1000,50,0.1,1.5$ & 15.8 & 15.8 & $1000,1000,50,0.2,2$ & 1.5 & 1.5 \\
$10,2000,25,0.01,1.1$ & 243 & 241.9 & $20,2000,25,0.05,1.2$ & 114.3 & 110.7 & $50,2000,50,0.1,1.5$ & 15.6 & 15.5 \\
$100,5000,25,0.05,1.2$ & 72.5 & 72.9 & $200,5000,50,0.1,1.5$ & 15.7 & 15.7 & $1000,5000,50,0.2,2$ & 1.6 & 1.5 \\
$10,10000,25,0.01,1.1$ & 214.3 & 215.9 & $20,10000,25,0.05,1.2$ & 109.1 & 105.4 & $50,10000,50,0.1,1.5$ & 15.7 & 15.5 \\
\hline
\end{tabular}

